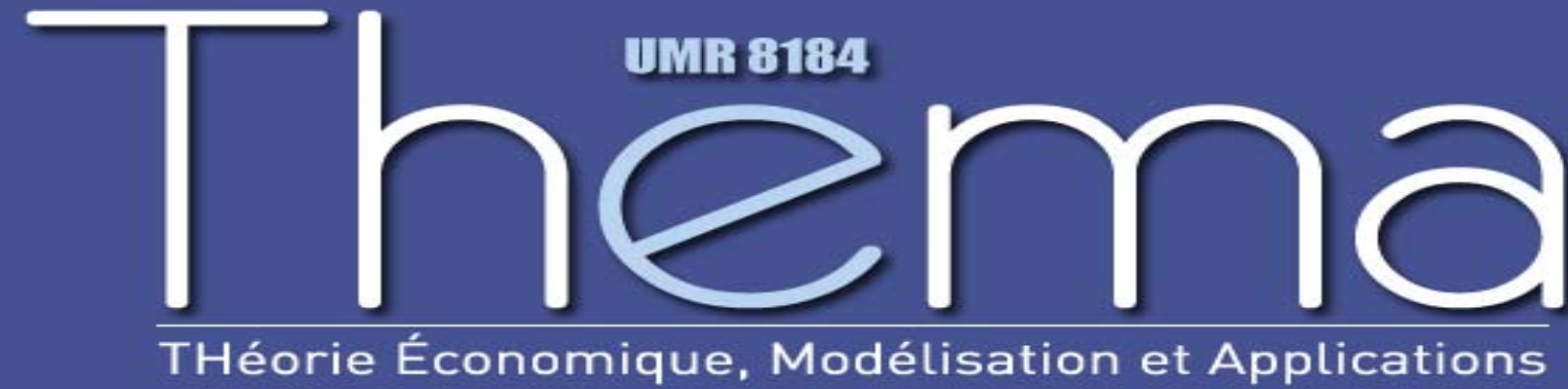

Thema Working Paper $n^{\circ} 2008-31$ Université de Cergy Pontoise, France

Réforme de la protection de l'emploi et inégalités face au chômage dans un moduele d'appariement

Olivier Charlot

Franck Malherbet

December 2008 



\title{
Réforme de la protection de l'emploi et inégalités face au chômage dans un modèle d'appariement*
}

\author{
Olivier Charlot Franck Malherbet $^{\ddagger}$
}

Octobre 2008

Résumé Cet article s'intéresse aux effets liés à l'introduction d'un système de modulation des cotisations patronales à l'assurance chômage (ou experience rating) sur le niveau et la structure du chômage par qualification. Nous construisons pour cela un modèle d'appariement dans lequel l'évolution de la demande de travail, les décisions de création et destruction d'emplois, ainsi que l'évolution des taxes destinées à financer l'assurance chômage sont endogènes. Dans ce cadre, la protection de l'emploi a des effets qui peuvent être différenciés selon le niveau de qualification considéré. L'introduction d'un système de modulation des cotisations employeur à l'assurance chômage pourrait améliorer le fonctionnement du marché du travail; l'importance des éventuels effets indésirables liés à ce système dépend à court terme de la capacité à substituer la taxe d'experience rating aux dispositifs de protection de l'emploi déjà en place, tandis qu'à plus long terme la hausse des incitations à se qualifier réduit en partie l'importance de ce problème.

Classification JEL J20; J60;

Mots Clés Destruction d'emplois; Coûts de licenciement; Experience rating

\footnotetext{
*Nous remercions Pierre Cahuc, Bruno Decreuse, Pierre Granier, Olivier L'Haridon et Benoit Lorel, ainsi que les participants au séminaire marché du travail à EUREQua, ceux du groupe de travail Macroéconomie du GREQAM pour leurs commentaires et suggestions avisés sur une version antérieure de cet article. Nous remercions également deux rapporteurs anonymes de cette revue pour l'ensemble de leurs remarques. Les éventuelles erreurs ou omissions résiduelles demeurent de notre responsabilité.

${ }^{\dagger}$ Université de Franche-Comté, LIBRE et CIRPEE. UFR SJEPG, 45d, Avenue de l'observatoire, 25030 Besançon Cedex. Courriel : ocharlot@univ-fcomte.fr

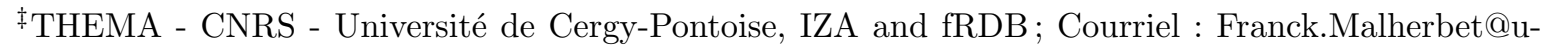
cergy.fr
} 


\section{Introduction}

La réforme de la législation sur la protection de l'emploi (LPE) est au coeur du débat de politique économique européen. En témoigne par exemple la volonté exprimée par la Commission Européenne d'inciter les Etats membres à "réexaminer, et le cas échéant, réformer les conditions trop restrictives de la législation en matière d'emploi qui affectent la dynamique du marché du travail et l'emploi des groupes confrontés à des difficultés pour accéder à ce marché [...]". Paradoxalement, la question de savoir ce que devrait être une bonne réforme de la LPE ne semble avoir qu'assez peu mobilisé le débat public puisqu'en dépit de son importance les gouvernements semblent avoir, jusque là, privilégié le statu quo ou les réformes à la marge moins coûteuses politiquement (Saint-Paul, 2002, OCDE, 2004, 2005, Boeri et Garibaldi, 2006).

Dans le cas particulier de la France, cette question a reçu récemment un écho favorable suite à la publication d'une série de rapports (Blanchard et Tirole, 2003, Cahuc, 2003, Camdessus, 2004) qui préconisent une refonte substantielle des dispositifs actuels de LPE à travers notamment : (i) une simplification des procédures administratives de licenciement et une diminution du rôle des instances judiciaires dans le processus de licenciement ; (ii) une responsabilisation accrue des entreprises dans leur mode de gestion de la maind'oeuvre grâce à une fiscalité adéquate. Ce dernier point s'inspire pour partie des systèmes d'experience rating (ou de modulation des cotisations patronales à l'assurance chômage) pratiqués aux Etats-Unis. Recommandé par l'OCDE (OCDE, 1994) et envisagé, dans un contexte différent, par deux rapports sur les prélèvements sociaux (Malinvaud, 1998 et Fougère et Margolis, 2000), ce système prévoit de taxer les licenciements proportionnellement au montant attendu des prestations que la caisse d'assurance chômage devra verser au salarié licencié ${ }^{1}$. La taxe sur les licenciements est ainsi indexée sur le coût induit par la décision de licenciement de l'entreprise pour le système d'assurance chômage. Un tel système a ainsi pour avantage de responsabiliser, au moins en partie, les entreprises quant au coût social de leur politique de licenciement, et pourrait ainsi améliorer l'efficacité et la cohérence de la LPE. De surcroît, il pourrait également s'avérer un puissant outil pour lutter contre les tensions récurrentes qui s'exercent sur l'équilibre financier des régimes d'assurance chômage.

Si l'introduction d'une taxe sur les licenciements inspirée de l'experience rating ne

1. Ce mode de financement des indemnités est une caractéristique unique du système d'assurance chômage américain, et est absent de tous les autres pays de l'OCDE où les prestations chômage sont essentiellement financées par des cotisations à taux uniforme payées par les employeurs ou les salariés, ou par des contributions gouvernementales (Holmlund, 1998). Il convient toutefois de noter qu'il peut exister des modalités spécifiques de financement dans certains pays, à l'instar de la contribution Delalande en France. Cette dernière devrait cependant être progressivement et définitivement supprimée à l'échéance du plan senior en 2010 . 
semble pas dépourvue d'avantages, il semble toutefois légitime de s'interroger sur l'impact de son introduction dans une économie comme celle de la France où l'emploi peu qualifié : (i) est déjà soumis à de fortes contraintes du fait des rigidités du marché du travail (ii) a déjà été très fortement affecté au cours des dernières décennies par une série d'évolutions défavorables de la demande de travail, (iii) s'avère davantage sensible aux modifications de la taxation et des rigidités institutionnelles que ne l'est l'emploi qualifié ( $c f$. par exemple Phelps, 1994, Drèze et Sneessens, 1997, Nickell et Bell, 1997, Malinvaud, 1998, ou encore Esping-Andersen, 2000).

Il est en effet bien établi que le chômage n'affecte pas de manière uniforme l'ensemble de la population, mais stigmatise au contraire certaines catégories sensibles, tout particulièrement les moins diplômés (OCDE, 1996, OCDE, 2004). Le tableau suivant rappelle l'importance des inégalités face au chômage :

Taux de Chômage (en \%) par qualification en France, moyenne sur la période 1994-2004, D'apres données OCDE Niveau éducatif Supérieur Secondaire Infra-Secondaire

$$
\text { Taux de Chômage } \quad 6,0 \quad 8,8 \quad 13,7
$$

Il apparaît que sur la période 1994-2004, le taux de chômage des individus diplômés de l'enseignement supérieur atteignait $6 \%$, il était donc plus de deux fois plus faible que celui des individus disposant d'un niveau d'éducation inférieur à l'enseignement secondaire $(13,7 \%)$. D'autre part, comme le soulignent Fougère et Margolis (2000) :

"Face au risque de chômage de (très) longue durée, plus élevé en France qu'aux Etats-Unis, les employeurs français pourraient en effet être amenés, dans le cadre d'un système d'experience rating, à se montrer plus sélectifs que leurs homologues américains au moment de l'embauche. [...] Un tel comportement pourrait avoir comme conséquence un accroissement du chômage de longue durée et des effets de stigmatisation qui lui sont associés"

S'inquiéter des effets induits par une taxe sur les licenciements sur les comportements d'embauche des entreprises, et par suite, sur la durée du chômage semble particulièrement important en ce qui concerne les travailleurs les moins qualifiés, déjà affectés par un taux de chômage important. Plus précisément, ceux-ci font face à des durées de chômage élevées, ainsi qu'à de forts taux de destruction. En indexant les taxes de licenciement sur le coût attendu pour l'assurance chômage, il est possible que ce système stigmatise ceux dont la durée d'indemnisation est potentiellement importante. Le résultat pourrait alors être d'aggraver le problème lié au chômage de longue durée auquel les non qualifiés sont déjà confrontés, même si l'effet sur leur taux de chômage peut être ambigu.

Dans cet article, nous montrons que l'allongement de la durée du chômage pour les moins qualifiés suite à l'instauration de la taxe d'experience rating n'est qu'éventuel, et dépend de la possibilité de pouvoir substituer experience rating et LPE traditionnelle. 
Partant, nous cherchons à évaluer les effets de l'introduction d'une taxe sur les licenciements, inspirée de l'experience rating américaine, sur le niveau, la durée ainsi que la composition du chômage par niveau de qualification, dans la lignée des travaux de Millard et Mortensen (1997) et Albrecht et Vroman (1999). Notre étude s'appuie sur un modèle d'équilibre du marché du travail à la Mortensen et Pissarides prenant en compte l'hétérogénéité de la main-d'oeuvre, différentes composantes de la LPE et le financement de l'assurance chômage, ainsi qu'une source d'hétérogénéité spécifique à la relation d'emploi. Dans ce cadre, nous étudions (i) les effets de la protection de l'emploi qui peuvent être différenciés en fonction du niveau de qualification des individus; (ii) l'impact de l'introduction d'un système d'experience rating sur le chômage agrégé, ainsi que sur la composition du chômage par niveau de qualification; (iii) les effets d'une réforme de la LPE dont la modalité est une substitution parfaite entre les procédures administratives et légales de licenciement et une taxe sur les licenciements (iv) et finalement, l'impact de la LPE sur les incitations à se qualifier.

Ces trois étapes nous permettent ainsi de compléter la littérature qui s'intéresse aux effets de la LPE sur la structure de l'emploi par qualification, de différencier les effets de la LPE traditionnelle de ceux liés à la taxe sur les licenciements, de montrer que les éventuels effets indésirables liés à la taxe d'experience rating dépendent du degré de substitution entre ce dispositif et la LPE existante, et enfin de montrer que les éventuels effets indésirables de la LPE pour les non qualifiés peuvent être contrebalancés par la hausse des incitations à s'éduquer, au moins dans le long terme, quand la structure par qualification de la population répond aux changements de la LPE.

L'article s'organise de la manière suivante : la section 2 présente la littérature reliée au problème que nous traitons. La section 3 présente le cadre d'analyse et les principales hypothèses afférentes à nos choix de modélisation. La section 4 étudie les principales propriétés de notre modèle à taxation donnée. La sections 5 s'intéresse aux effets de la LPE sur la productivité moyenne des emplois et la structure par qualification de la population active, avant de procéder en section 6 à l'analyse des résultats de nos exercices de politique économique lorsque l'endogénéité de la taxation est prise en compte. En section 7, nous discutons l'impact d'hypothèses de modélisation alternatives sur nos résultats. Enfin, la section 8 conclut.

\section{Littérature reliée}

Ce travail s'inscrit pour partie dans la perspective des travaux de Mortensen et Pissarides (1999, 2003). Il ressort de leurs travaux que si la LPE a un effet sur le chômage qui est a priori incertain, elle conduit à un allongement de la durée des épisodes de chômage, ainsi qu'à une aggravation importante des problèmes d'employabilité pour les moins qua- 
lifiés. Dans sa forme actuelle, la LPE serait un facteur important contribuant à expliquer les différences de performance entre les marchés du travail américain et européen. Les exercices numériques réalisés par ces auteurs suggèrent également qu'elle affecte plus particulièrement de façon négative les non qualifiés. Plus précisément, les effets de la LPE sur la durée du chômage et l'employabilité semblent assez robustes. En revanche, il n'existe pas à notre connaissance de consensus quant à son effet sur le chômage : les résultats numériques tirées des modèles théoriques diffèrent généralement, notamment selon la forme du processus de négociation salariale envisagé (e.g. Mortensen et Pissarides, 1999b, ou Ljungqvist, 2002), ou encore suivant le caractère flexible ou non des salaires (Cahuc et Zylberberg, 1999, Garibaldi et Violante, 2004). Cette ambiguïté se retrouve dans les évaluations empiriques qui ne dégagent pas de résultats très tranchés quant à l'effet de la LPE sur le taux de chômage (OCDE, 2004).

Notre étude complète ces travaux en introduisant dans un cadre proche de nouveaux éléments susceptibles d'affecter les flux sur le marché du travail, notamment une taxe sur les licenciements inspirée de l'experience rating américaine, dont le montant est indexé sur la durée espérée des épisodes de chômage et dont l'objet est de financer la caisse d'assurance chômage.

Que peut-on attendre de l'introduction d'une taxe sur les licenciements? Dans la mesure où ce système n'est pas en vigueur en Europe, il peut sembler difficile d'en prévoir les effets. On peut cependant tirer parti des études empiriques disponibles dans le cas américain, ainsi que des travaux théoriques sur le sujet.

Depuis les contributions originales de Feldstein (1976) ou encore Topel et Welsh (1980), il existe une littérature abondante sur l'analyse économique des effets de l'experience rating $^{2}$. Le raisonnement en faveur de ce système est simple. En l'absence d'experience rating (ou lorsqu'elle est imparfaite), les entreprises lorsqu'elles licencient ne supportent individuellement qu'une partie du coût des indemnités de chômage versées à leurs anciens salariés. Il existe donc une distorsion dans les décisions de licenciement puisque les firmes n'internalisent pas (ou imparfaitement) le coût fiscal de leur politique de gestion de la main-d'oeuvre, ce qui se traduit par une rotation plus importante des effectifs. Ces auteurs recommandent alors l'utilisation d'un mécanisme fiscal incitatif afin de décourager les licenciements, i.e. l'adoption d'un système d'experience rating parfait où les entreprises supportent individuellement la totalité des indemnités de chômage versées à leur anciens employés. Le passage à un tel système induirait alors une baisse significative du chômage. Ces conclusions théoriques sont critiquées par Burdett et Wright (1989), Marceau (1993) ou encore plus récemment par Mongrain et Roberts (2005). Selon ces auteurs, le passage à un système parfait serait susceptible d'accroître le taux de chômage. D'un point de vue empirique cependant, les études menées sur données américaines tendent à confirmer

2. Pour un survol de la littérature, voir par exemple, Fougère et Margolis (2000) ou Malherbet (2003). 
les conclusions de Feldstein (1976) et attestent des effets bénéfiques de ce système sur l'emploi aux États-Unis (voir par exemple Card et Levine, 1994 ou encore Anderson et Meyer, 2000).

Comme le montre une partie des études empiriques, ceci est en grande partie lié au fait qu'aux Etats-Unis, plusieurs secteurs sont en permanence subventionnés dans le financement de l'assurance chômage : les secteurs qui emploient des travailleurs relativement peu qualifiés - typiquement, le secteur minier ou celui de la construction - sont subventionnés dans la plupart des Etats par les secteurs employant des travailleurs plus qualifiés comme la finance ou les services. Ceci permet aux secteurs les moins stables de se développer par rapport à d'autres secteurs plus stables. Ainsi, Deere (1991) montre à partir de données provenant de 31 Etats américains et 7 secteurs d'activité sur la période 1962-1969, qu'une hausse de $10 \%$ de la subvention implicite accroît l'emploi de 1,7\% dans la construction et diminue l'emploi de 1\% dans les services. La suppression de ce subventionnement, due au passage à un système d'experience rating parfait tendrait à stabiliser la main-d'oeuvre et accroître l'emploi.

Ces conclusions peuvent être complétées par celles issues de travaux théoriques récents, au nombre desquels Fath et Fuest (2005), Mongrain et Roberts (2005) et Cahuc et Malherbet (2004) qui étudient dans des cadres différents l'impact de l'experience rating sur les plans positifs et normatifs.

Fath et Fuest (2005) comparent les effets des indemnités de licenciement (transfert de l'entreprise vers les travailleurs) et de l'experience rating dans un modèle de salaire d'efficience où les agents sont hétérogènes et où les destructions d'emplois et le contrôle des travailleurs sont endogènes. Ils montrent que l'introduction d'une taxe d'experience rating réduit le chômage et accroît le bien-être. La logique de ce modèle est simple : une hausse de la taxe sur les licenciements tend à réduire, ceteris paribus, le contrôle de l'entreprise sur les travailleurs puisque cette activité est coûteuse. Toutefois, la hausse de cette taxe induit également une réduction des cotisations sociales assises sur la masse salariale. Par ailleurs, elle n'affecte pas l'effort des travailleurs puisque contrairement aux indemnités de licenciement la taxe d'experience rating ne s'apparente pas à un transfert de la firme vers les travailleurs. Toujours dans le cadre d'un modèle de salaire d'efficience, mais dans un cadre statique cette fois, Mongrain et Roberts (2005) montrent que lorsque les entreprises versent des indemnités de licenciement aux travailleurs, une variation du degré d'experience rating peut réduire ces indemnités d'un montant suffisant pour faire baisser le bien-être des travailleurs. Comparée à ces modèles, notre étude se concentre sur des aspects positifs plutôt que normatifs, et cherche plus particulièrement à examiner dans quelle mesure LPE et experience rating sont susceptibles d'avoir des effets différenciés pour les qualifiés et les non qualifiés. Notre cadre d'étude diffère de ces modèles en prenant en compte les frictions d'appariement sur le marché du travail dans un cadre intertemporel 
où la main-d'oeuvre est hétérogène, mais n'incorpore pas d'asymétrie d'information. Nous considérons une autre composante de la LPE, les procédures administratives et judiciaires de licenciement, dont l'importance a été soulignée à de nombreuses reprises ${ }^{3}$ (Blanchard, 2000, Kramarz et Michaud, 2004).

De par son cadre d'analyse, l'article le plus proche du notre est celui de Cahuc et Malherbet (2004), qui s'interroge sur les effets de l'introduction d'un système d'experience rating au regard des spécificités institutionnelles inhérentes aux marchés du travail d'Europe Continentale. Dans cette optique les auteurs développent un modèle d'équilibre avec deux types d'emplois (CDI/CDD) sur un segment particulier du marché du travail, celui des travailleurs non qualifiés dont le pouvoir de négociation est par hypothèse faible. Dans ce modèle, les travailleurs sont averses au risque mais les employeurs disposent de tout le pouvoir de négociation de telle sorte que les employés sont payés soit au niveau de leur utilité de réserve, soit au niveau d'un salaire minimum contraignant. Les auteurs montrent alors que l'introduction d'un système d'experience rating tend à augmenter l'emploi et le bien-être dans l'économie. Notre modèle se démarque du cadre envisagé par Cahuc et Malherbet sur deux points principaux : (i) les agents sont neutres au risque mais disposent en contrepartie d'un pouvoir de négociation qui n'est pas nécessairement nul. Cette hypothèse est importante dans la mesure où la négociation permet d'internaliser une partie des effets liés aux rigidités sur le marché du travail ${ }^{4}$. (ii) Nous considérons un modèle dual alors que leur article se concentre sur le segment non qualifié du marché du travail. D'une part, ceci permet d'analyser les effets de l'experience rating et de la LPE sur les incitations à se qualifier. Les effets de l'experience rating peuvent ainsi différer selon que la proportion de travailleurs qualifiés est exogène ou endogène. D'autre part, ceci nous permet de tenir compte des interactions qui peuvent lier qualifiés et non qualifiés à travers la modification du mode de financement de l'assurance chômage. Ces interactions peuvent être importantes dans la mesure où comme le souligne la littérature empirique évoquée précédemment (e.g. Deere, 1991) certains secteurs employant majoritairement des travailleurs peu qualifiés sont subventionnés de manière permanente par les secteurs

3. Nous ne prenons pas en compte les indemnités versées aux travailleurs en cas de séparation pour au moins deux raisons. La première, évoquée dans le texte, indique que les coûts administratifs dépassent largement la valeur monétaire des indemnités de licenciement. La seconde a trait à la critique de Lazear (1990), dite Bonding Critique. Il montre en présence de contrats complets que la négociation salariale entre l'entreprise et le travailleur annihile tout effet des indemnités de licenciement sur les décisions des agents, i.e. seul le profil des salaires est alors affecté. Le salaire d'embauche diminue afin de compenser le versement ultérieur des indemnités de licenciement qui s'apparentent alors à un élément de rémunération inclu dans le contrat de travail. Ceci implique qu'elles n'ont pas d'influence sur l'équilibre du marché du travail lorsque le salaire est librement négocié.

4. Nous revenons sur ce point dans l'avant dernière section de notre article afin de mettre en perspective nos résultats avec ceux de Cahuc et al. (2004) lorsqu'il existe un salaire minimum contraignant dans l'économie. 
employant essentiellement des travailleurs qualifiés. Pour cette raison, nous accordons une attention particulière à ce phénomène dans les développements qui suivent.

\section{Le modèle}

Le modèle proposé s'appuie sur le cadre analytique élaboré par Mortensen et Pissarides (1994), étendu de manière à intégrer les trois éléments suivants : (i) une source d'hétérogénéité spécifique à l'appariement, liée à l'existence d'un aléa dans la qualité de l'appariement, (ii) une source d'hétérogénéité spécifique à la main-d'oeuvre, liée à l'existence de compétences intrinsèques ou de qualifications hétérogènes dans la population, et enfin, (iii) des coûts d'ajustement liés à l'existence de procédures administratives de licenciement et des taxes sur les licenciements.

\subsection{Hypothèses préliminaires}

Le temps est continu. A chaque instant, $\delta>0$ agents naissent et entrent sans délai au chômage. Chaque individu fait face à un risque de décès constant $\delta$, de sorte que la taille de la population est stationnaire et peut être normalisée à l'unité. Les agents sont neutres au risque ${ }^{5}$ et $\rho$ désigne le taux de préférence pour le présent, ainsi que le taux d'intérêt de l'économie. On note $r=\rho+\delta$ le taux d'escompte effectif de cette économie.

La population est répartie en 2 groupes de qualification distincts ${ }^{6}$ coexistant en proportion $P_{1}=P$ et $P_{2}=1-P$. Nous prenons pour commencer ces proportions comme données; elles seront endogénéisées par la suite. Les individus de type $i=1,2$ sont ainsi dotés d'une caractéristique productive $s_{i}$; les deux groupes peuvent être hiérarchisés en fonction de cette caractéristique, i.e. $s_{1} \geq s_{2}$. Ces travailleurs de types différents effectuent leur recherche d'emploi sur des marchés séparés en fonction de leur niveau de qualification ${ }^{7}$. Les rencontres sur chaque marché se réalisent par l'intermédiaire d'un pro-

5. La linéarité de la fonction d'utilité n'est pas une hypothèse trop restrictive dans la mesure où nous nous limitons à une analyse positive. Nous ne tenons cependant pas compte des effets assurantiels de ces dispositifs, étudiés par exemple par Pissarides (2001), Bertola (2004), Blanchard et Tirole (2008) ou Cahuc et Zylberberg (2008). Nous postulons également la linéarité pour les entreprises, un point sur lequel nous revenons dans la dernière section de cet article.

6. Le choix de se limiter à deux groupes est fait pour faciliter la présentation des résultats. Rien n'empêche cependant de prendre en compte un nombre de marchés plus grand.

7. Cette hypothèse peut être parfaitement justifiée dans la mesure où la qualification/l'éducation d'un individu est une caractéristique facilement observable, généralement spécifiée dans les offres d'emploi. La segmentation complète des marchés n'empêche pas l'existence d'interactions entre niveaux de qualification, via la taxation, comme le montrent les développement qui suivent. Ces interactions qui transitent par la taxation subsistent dans les cas où les marchés sont segmentés de manière imparfaite, par exemple lorsque les qualifiés acceptent des emplois non qualifiés, tout en continuant éventuellement la recherche d'emplois qualifiés. Notons enfin qu'en l'absence totale de segmentation, les taux d'entrée et sortie du 
cessus imparfait, capturé par la fonction d'appariement habituelle qui relie le nombre total de rencontres $M_{i}$ qui se produisent sur un segment donné du marché au nombre de protagonistes de chaque côté de ce marché, i.e. $M_{i} \equiv M_{i}\left(U_{i}, V_{i}\right)$ où $U_{i}$ est le nombre (masse) de chômeurs de type $i$ recherchant de manière active un emploi sur le marché, et $V_{i}$ représente le nombre de postes vacants sur ce même marché. La technologie d'appariement à l'oeuvre sur chaque marché satisfait aux hypothèses usuelles : croissante, continûment différentiable et homogène de degré 1 , vérifiant les conditions aux bornes $M_{i}\left(0, x_{1}\right)=M_{i}\left(x_{2}, 0\right)=0$ pour tout $x_{1}, x_{2} \geq 0$, ainsi que les conditions d'Inada. L'homogénéité de degré 1 de cette fonction nous permet d'écrire la probabilité qu'un emploi vacant rencontre un travailleur comme une fonction de la tension du marché du travail $\theta_{i}$, i.e. $M_{i}\left(U_{i}, V_{i}\right) / V_{i}=M_{i}\left(U_{i} / V_{i}, 1\right)=m_{i}\left(\theta_{i}\right)$ avec $m_{i}^{\prime}\left(\theta_{i}\right) \leq 0$. De même le taux de contact pour un travailleur donné s'écrit $M_{i}\left(U_{i}, V_{i}\right) / U_{i}=\theta_{i} m_{i}\left(\theta_{i}\right)$, où $\theta_{i} m_{i}\left(\theta_{i}\right)$ est une fonction croissante de $\theta_{i}$.

Sur chaque marché, il existe un continuum de firmes dont le nombre est endogène à l'équilibre, produisant un unique bien et utilisant le travail comme seul intrant. Chaque firme est dotée d'une technologie à coefficient constant, requérant un travailleur de type $i$ pour produire $y_{i} \equiv s_{i}+\varepsilon$ unités de biens, où $\varepsilon$ est une variable aléatoire caractérisant la qualité de l'appariement, tirée à partir d'une distribution stationnaire et connue de tous, $G_{i}$, sur le support $\left[\varepsilon_{l_{i}}, \varepsilon_{u_{i}}\right]$. Partant de l'idée que les emplois peu qualifiés ont une probabilité plus grande d'obtenir une faible productivité que les emplois qualifiés, nous supposons par la suite que $G_{1}(\varepsilon)<G_{2}(\varepsilon)$ quel que soit $\varepsilon$, de sorte que la première distribution est stochastiquement dominée par la deuxième. Les emplois débutent avec un niveau de productivité spécifique à l'appariement $\varepsilon$ aléatoire ${ }^{8}$. Plus précisément, cette hypothèse implique que toutes les rencontres ne déboucheront pas nécessairement sur une embauche : dès lors qu'il existe des rigidités sur le marché du travail, le surplus attendu de certaines rencontres peut s'avérer négatif. Ceci sera le cas lorsque la productivité d'un appariement est trop faible en regard des coûts qu'engendreraient la création d'emplois. Le surplus qui résulte d'une rencontre augmente néanmoins avec le paramètre de productivité spécifique au travailleur, et a ainsi d'autant plus de chances d'être positif que ce paramètre est élevé ${ }^{9}$. Ceci implique qu'à mesure que l'on monte dans l'échelle des qualifications, les individus sortent en moyenne plus rapidement du chômage, puisque les rencontres entre les travailleurs et les firmes ont davantage de chances de déboucher sur une relation d'emploi profitable. Les emplois existants sont, quant à eux, soumis à un risque $\lambda_{i}$ d'être frappé par un choc de productivité, qui consiste en un nouveau tirage du

chômage seraient ici tout de même différenciés par qualification.

8. Nous supposons par souci de simplicité que la valeur initiale de la productivité $\varepsilon$ résulte d'un tirage sur la distribution $G_{i}$. Pour plus de détails, voir Pissarides (2000), chapitre 6 .

9. Ceci sera du moins le cas dès lors que les rigidités ne croissent pas (ou pas trop) avec la qualification des individus. 
paramètre $\varepsilon$ sur la distribution $G_{i}$. Ces chocs impliquent que le surplus associé à un emploi peut varier et éventuellement devenir négatif ; la relation d'emploi prendra fin dans cette éventualité. Le surplus engendré par un travailleur plus qualifié étant plus important, il faudra un choc d'ampleur plus grande pour que l'emploi soit détruit. Les travailleurs plus qualifiés bénéficient donc non seulement d'un avantage en terme de durée du chômage, mais également en terme de durée de la relation d'emploi par rapport à des travailleurs de moindre niveau d'éducation ${ }^{10}$. De la sorte, décisions d'embauche et de licenciement sont endogènes; elles sont liées au niveau de qualification des individus, et répondent aux changements du contexte institutionnel, ce qui fait de ce cadre d'étude un outil adéquat pour évaluer l'impact de diverses mesures de protection de l'emploi et de financement de l'assurance chômage, tant sur l'évolution du chômage en termes agrégés, que sur les performances individuelles des travailleurs sur le marché du travail, ou encore sur les inégalités de chômage.

Toute relation d'emploi peut prendre fin au prix d'un coût de séparation $f_{i}+\tau_{e_{i}}$ supporté par la firme. La première composante, $f_{i}$, représente une composante traditionnelle de la LPE. Elle s'apparente aux procédures administratives et judiciaires de licenciement et recouvre l'ensemble des coûts irrévocables dépensés par exemple en action en justice lors d'un licenciement ou lors des procédures de reclassement interne. La seconde composante, $\tau_{e_{i}}$, représente une taxe sur les licenciements ou taxe d'experience rating dont les caractéristiques sont les suivantes ${ }^{11}$ : (i) elle affecte les décisions de licenciement des entreprises; (ii) elle sert à financer les indemnités de chômage versées aux chômeurs ; (iii) elle est indexée sur la durée espérée des épisodes de chômage.

\subsection{Surplus, gains des agents et négociation salariale}

Un emploi peut se trouver dans l'une des trois situations suivantes : vacant, nouvellement pourvu ou existant. On note $\Pi_{v_{i}}, \Pi_{o_{i}}(\varepsilon)$ les valeurs respectives d'un emploi vacant et nouvellement pourvu, $\Pi_{e_{i}}(\varepsilon)$ la valeur d'un emploi pourvu existant. De même, on note également $V_{u_{i}}$ et $V_{o_{i}}(\varepsilon)$ les utilités respectives attendues de la recherche d'un emploi et de

10. Cette propriété est conforme à ce qui ressort des études microéconométriques, où le niveau d'éducation affecte positivement la durée des épisodes d'emploi et/ou négativement la durée des épisodes de chômage (voir notamment Nickell, 1979, Mincer, 1991, Devine et Kieffer, 1991, Cohen, Lefranc et Saint-Paul, 1997, Givord et Maurin, 2004).

11. Il convient toutefois de noter que les systèmes en vigueur aux Etats-Unis sont plus complexes que la modélisation retenue dans ce papier (voir par exemple Malherbet, 2003). En particulier, la taxe d'experience rating est payée au cours du temps plutôt qu'au moment du licenciement. La prise en compte de ce phénomène ajoute une complexité considérable au modèle. En conséquence, nous supposons un processus sans mémoire. Cette hypothèse est commune à tous les modèles dynamique consacrés à l'analyse des effets de l'experience rating (voir par exemple, Card et al., 1994, Albrecht et al., 1999, Cahuc et al., 2004 ou encore Fath et al., 2005). 
la détention d'un emploi nouvellement pourvu, et $V_{e_{i}}(\varepsilon)$, l'utilité attendue de la détention d'un poste existant.

La raison de la distinction entre postes nouvellement créés et postes existants tient aux coûts de licenciement, dont l'effet dans la négociation salariale diffère au moment de l'embauche et lors des renégociations ultérieures. La négociation qui s'opère au moment de la rencontre se déroule entre une firme et un individu qui n'est pas encore embauché et qui ne bénéficie donc encore pas de la LPE. Il n'y aura ainsi pas à proprement parler de licenciement en cas de désaccord dans la négociation, chacun étant libre de retourner sur le marché des appariements à la recherche d'un partenaire. Le surplus initial $S_{o_{i}}$ est donc défini par

$$
S_{o_{i}}(\varepsilon)=\Pi_{o_{i}}(\varepsilon)-\Pi_{v_{i}}+V_{o_{i}}(\varepsilon)-V_{u_{i}}
$$

Les choses sont, en revanche, différentes en ce qui concerne les salaires négociés ultérieurement en cas de choc. Une fois le travailleur embauché, l'employeur tombe sous le coup de la LPE qui impose de payer des coûts de licenciement en cas de désaccord dans la négociation. Les coûts de licenciement affectent donc directement le surplus $S_{e_{i}}(\varepsilon)$ d'un emploi existant, qui s'écrit

$$
S_{e_{i}}(\varepsilon)=\Pi_{e_{i}}(\varepsilon)-\Pi_{v_{i}}+f_{i}+\tau_{e_{i}}+V_{e_{i}}(\varepsilon)-V_{u_{i}}
$$

Les salaires sont négociés selon un processus de marchandage à la Nash, qui partage le surplus associé à l'emploi en proportions fixes pour la firme et le travailleur. La différence entre les surplus (1) et (2) implique que pour un niveau de qualification donné $s_{i}$, les négociations aboutissent à des salaires différenciés selon le statut de l'emploi, et que nous noterons respectivement $w_{o_{i}}(\varepsilon)$ et $w_{e_{i}}(\varepsilon)$ pour un salaire à l'embauche et pour un salaire dans la continuité de la relation d'appariement. Ces salaires sont solutions des programmes qui suivent :

$$
\begin{aligned}
\max _{w_{o_{i}}} & <\beta_{i} \ln \left(V_{o_{i}}(\varepsilon)-V_{u_{i}}\right)+\left(1-\beta_{i}\right) \ln \left(\Pi_{o_{i}}(\varepsilon)-\Pi_{v_{i}}\right)> \\
\max _{w_{e_{i}}} & <\beta_{i} \ln \left(V_{e_{i}}(\varepsilon)-V_{u_{i}}\right)+\left(1-\beta_{i}\right) \ln \left(\Pi_{e_{i}}(\varepsilon)-\Pi_{v_{i}}+f_{i}+\tau_{e_{i}}\right)>
\end{aligned}
$$

Les différentes valeurs satisfont alors les équations d'arbitrage suivantes. La détention d'un emploi vacant induit un coût de recherche $c_{i}>0$ payé continûment et sa valeur est donnée par l'équation d'arbitrage suivante :

$$
\rho \Pi_{v_{i}}=-c_{i}+m_{i}\left(\theta_{i}\right)\left[\int_{\varepsilon_{l_{i}}}^{\varepsilon_{u_{i}}} \operatorname{Max}\left[\Pi_{o_{i}}(\zeta), \Pi_{v_{i}}\right] d G_{i}(\zeta)-\Pi_{v_{i}}\right]
$$

Tout emploi vacant est susceptible de rencontrer un travailleur au taux $m_{i}\left(\theta_{i}\right)$. Cette rencontre n'aboutit à une création d'emploi qu'à condition d'engendrer un gain pour la firme. Il existe une source d'incertitude sur la productivité de l'emploi qui sera créé, 
chaque emploi nouveau étant soumis à un aléa sur la valeur de la productivité spécifique à l'appariement $\varepsilon$. Pour cette raison, le gain en capital attendu par une firme lorsque se produit une rencontre est considéré en termes d'espérance par rapport à cette variable aléatoire dans l'équation (4).

Les valeurs $\Pi_{o_{i}}(\varepsilon)$ et $\Pi_{e_{i}}(\varepsilon)$ associées à la détention d'un emploi nouvellement créé ou pourvu avec un travailleur de type $s_{i}$ et dont la valeur du paramètre de productivité spécifique à l'appariement est $\varepsilon$ s'écrivent :

$$
\begin{aligned}
& r \Pi_{o_{i}}(\varepsilon)=\varepsilon+s_{i}-(1+\tau) w_{o_{i}}(\varepsilon)+\lambda_{i}\left[\int_{\varepsilon_{l_{i}}}^{\varepsilon_{u_{i}}} \operatorname{Max}\left[\Pi_{e_{i}}(\zeta), \Pi_{v_{i}}-f_{i}-\tau_{e_{i}}\right] d G_{i}(\zeta)-\Pi_{o_{i}}(\varepsilon)\right] \\
& r \Pi_{e_{i}}(\varepsilon)=\varepsilon+s_{i}-(1+\tau) w_{e_{i}}(\varepsilon)+\lambda_{i}\left[\int_{\varepsilon_{l_{i}}}^{\varepsilon_{u_{i}}} \operatorname{Max}\left[\Pi_{e_{i}}(\zeta), \Pi_{v_{i}}-f_{i}-\tau_{e_{i}}\right] d G_{i}(\zeta)-\Pi_{e_{i}}(\varepsilon)\right]
\end{aligned}
$$

Une relation d'emploi avec un travailleur de type $s_{i}$ offre un profit instantané égal à $\varepsilon+s_{i}-(1+\tau) w_{o_{i}}(\varepsilon)$ pour un emploi nouvellement créé et $\varepsilon+s_{i}-(1+\tau) w_{e_{i}}(\varepsilon)$ pour un emploi existant, i.e. la valeur courante de la productivité de la relation d'emploi, diminuée du salaire versé au travailleur et de la taxe acquittée par l'employeur qui correspond à une proportion $\tau$ de la masse salariale. Le paramètre de productivité spécifique à l'appariement $\varepsilon$ est également affecté par des chocs régis par un processus aléatoire qui suit une loi de Poisson de paramètre $\lambda_{i}$. Dans l'éventualité d'un choc, une nouvelle valeur du paramètre de productivité spécifique $\varepsilon$ est tirée de la distribution $G_{i}$. La firme compare alors la valeur associée à la destruction de l'emploi $\Pi_{v_{i}}-f_{i}-\tau_{e_{i}}$ au profit attendu de la poursuite de la relation d'emploi $\Pi_{e_{i}}(x)$, et lorsque la valeur espérée de ce profit est trop faible l'emploi est détruit.

L'utilité attendue $V_{u_{i}}$ par un individu de qualification $s_{i}$ en recherche d'emploi satisfait :

$$
r V_{u_{i}}=b_{i}+\theta_{i} m_{i}\left(\theta_{i}\right)\left[\int_{\varepsilon_{l_{i}}}^{\varepsilon_{u_{i}}} \operatorname{Max}\left[V_{o_{i}}(\zeta), V_{u_{i}}\right] d G_{i}(\zeta)-V_{u_{i}}\right]
$$

où $b_{i}$ désigne les subsides versés aux chômeurs de type $i$ et l'utilité que ceux-ci retirent de leurs loisirs. Un chercheur d'emploi entre en contact avec une firme au taux $\theta_{i} m_{i}\left(\theta_{i}\right)$ et réalise un gain en termes d'utilité attendue égal à $\int_{\varepsilon_{c_{i}}}^{\varepsilon_{u_{i}}} V_{o_{i}}(\zeta) d G(\zeta)-V_{u_{i}}$ si la rencontre est fructueuse.

Les valeurs $V_{o_{i}}(\varepsilon)$ et $V_{e_{i}}(\varepsilon)$ associées respectivement à l'emploi pour un travailleur de type $s_{i}$ employé sur un poste nouvellement créé ou existant dont la productivité spécifique est $\varepsilon$ s'écrivent :

$$
r V_{o_{i}}(\varepsilon)=w_{o_{i}}(\varepsilon)+\lambda_{i}\left[\int_{\varepsilon_{l_{i}}}^{\varepsilon_{u_{i}}} \operatorname{Max}\left[V_{e_{i}}(\zeta), V_{u_{i}}\right] d G_{i}(\zeta)-V_{o_{i}}(\varepsilon)\right]
$$




$$
r V_{e_{i}}(\varepsilon)=w_{e_{i}}(\varepsilon)+\lambda_{i}\left[\int_{\varepsilon_{l_{i}}}^{\varepsilon_{u_{i}}} \operatorname{Max}\left[V_{e_{i}}(\zeta), V_{u_{i}}\right] d G_{i}(\zeta)-V_{e_{i}}(\varepsilon)\right]
$$

Les travailleurs perçoivent un salaire instantané égal à $w_{o_{i}}(\varepsilon)$ et $w_{e_{i}}(\varepsilon)$ selon que le poste est nouvellement créé ou existant. Dans chacun des cas, la productivité est susceptible de changer selon le processus de Poisson décrit précédemment, dans une telle éventualité le travailleur compare la nouvelle valeur associée à l'emploi à la valeur associée à la recherche d'emploi $V_{u_{i}}$.

\subsection{Règles de décision}

Seuil de création. Une rencontre aboutit à une création d'emploi dès lors qu'elle est profitable pour la firme et le travailleur, i.e. $S_{o_{i}}(\varepsilon) \geq 0$. Cette condition n'est pas nécessairement satisfaite du fait des subsides de l'assurance chômage versés aux chercheurs d'emploi, et des taxes supportées par les entreprises. Il existe ainsi pour chaque catégorie de qualification un seuil endogène $\varepsilon_{c_{i}}$ en deçà duquel les rencontres ne débouchent pas sur des créations d'emploi. Ce seuil est obtenu en saturant la contrainte, et correspond ainsi au niveau minimal de la productivité spécifique à l'appariement requise pour entamer de manière rentable une relation d'emploi avec un travailleur de qualification $s_{i}$, i.e. le seuil $\varepsilon_{c_{i}}$ est défini de manière implicite par :

$$
S_{o_{i}}\left(\varepsilon_{c_{i}}\right)=0
$$

Seuil de destruction. La poursuite d'une relation d'emploi est conditionnelle au fait que cette relation soit profitable pour toutes les parties, i.e. $S_{e_{i}}(\varepsilon) \geq 0$. De manière similaire au seuil de création, on peut définir un seuil de destruction $\varepsilon_{d_{i}}$ pour chaque catégorie de travailleur en deçà duquel l'appariement se termine. Ce seuil est définit en saturant la contrainte, puisqu'il correspond au niveau minimal de la productivité spécifique à l'appariement requise pour poursuivre une relation d'emploi. Formellement, nous avons :

$$
S_{e_{i}}\left(\varepsilon_{d_{i}}\right)=0
$$

Libre entrée. La création d'emploi est régie par une condition de libre entrée sur chaque marché. Cette hypothèse implique que de nouvelles firmes entrent sur chaque marché jusqu'à épuisement de toute rente, ce qui ramène la valeur d'un emploi vacant à zéro, i.e. :

$$
\Pi_{v_{i}}=0
$$

\subsection{Taux de chômage par qualification}

Dans ce modèle, des travailleurs hétérogènes bénéficient de performances différentes en termes de taux d'entrée et de sortie du chômage. En effet, le taux d'entrée au chômage 
d'un travailleur de niveau de qualification $s_{i}$ participant au marché $i$ est donné par :

$$
q_{i}=\lambda_{i} G_{i}\left(\varepsilon_{d_{i}}\right)
$$

Ce taux $q_{i}$ correspond de manière usuelle à la probabilité d'occurrence d'un choc de productivité $\lambda_{i}$, multipliée par la probabilité $G_{i}\left(\varepsilon_{d_{i}}\right)$ que le paramètre de productivité spécifique tombe en deçà d'une valeur seuil de productivité, i.e. la productivité de réservation à la destruction, notée $\varepsilon_{d_{i}}$. Le taux de sortie du chômage vérifie quant à lui :

$$
\mu_{i}=\theta_{i} m_{i}\left(\theta_{i}\right)\left[1-G_{i}\left(\varepsilon_{c_{i}}\right)\right]
$$

Ce taux $\mu_{i}$ correspond à la probabilité $\theta_{i} m_{i}\left(\theta_{i}\right)$ de réaliser une rencontre, multipliée par la probabilité $1-G_{i}\left(\varepsilon_{c_{i}}\right)$ que cette rencontre soit acceptable, i.e. que la productivité de réservation à l'embauche, notée $\varepsilon_{c_{i}}$, soit suffisante.

En notant respectivement $E_{i}$ et $U_{i}$ les masses totales de travailleurs de type $s_{i}$ en emploi et au chômage quel que soit leur âge, les flux sur chaque marché sont régis par :

$$
\begin{aligned}
& d U_{i} / d t=\delta P_{i}+q_{i} E_{i}-\left(\delta+\mu_{i}\right) U_{i} \\
& d E_{i} / d t=\mu_{i} U_{i}-\left(\delta+q_{i}\right) E_{i}
\end{aligned}
$$

Le flux des entrants au chômage est composé des $\delta P_{i}$ agents qui entrent dans la population de qualification $i$ et entament immédiatement leur recherche d'emploi, ainsi que de $q_{i} E_{i}$ agents qui viennent d'être licenciés. En contrepartie, $\left(\delta+\mu_{i}\right) U_{i}$ agents sortent du chômage, soit du fait de leur décès au taux exogène $\delta$, soit parce que leur recherche aboutit à une embauche, ce qui se produit de manière endogène au taux $\mu_{i}$. Les entrées en emploi correspondent aux $\mu_{i} U_{i}$ chercheurs d'emploi qui viennent d'être recrutés, tandis que les sorties en nombre $\left(\delta+q_{i}\right) E_{i}$ correspondent soit à des décès au taux $\delta$, soit à des licenciements qui interviennent de façon endogène au taux $q_{i}$. En notant $u_{i}=U_{i} / P_{i}$ le taux de chômage des individus de qualification $s_{i}$, on obtient à l'équilibre des flux :

$$
u_{i}=\frac{\delta+q_{i}}{\delta+q_{i}+\mu_{i}} \text { et } E_{i}=\left(1-u_{i}\right) P_{i}
$$

\subsection{Contrainte budgétaire et fiscalité}

Pour des raisons de solvabilité, le financement de l'indemnisation du chômage est soumis à une contrainte d'équilibre budgétaire. Il n'est donc pas possible de fixer indépendamment le montant des subsides versés aux chômeurs et les taxes qui servent à les financer. Deux types d'instruments peuvent être envisagés pour opérer ce financement : le taux de taxe, $\tau$, perçu sur la masse salariale de chaque secteur, et une taxe, $\tau_{e_{i}}$ versée par chaque entreprise lorsqu'elle licencie. Cette seconde taxe sur les licenciements ou taxe d'experience rating est dite parfaite lorsque $\tau=0$, i.e. lorsque les firmes qui licencient supportent 
individuellement l'intégralité du montant de la dépense qu'elles engendrent auprès de la caisse d'assurance chômage au travers de leur décision. Dans les autres cas, le système sera qualifié d'imparfait dans la mesure où le financement des indemnités de chômage est - au moins en partie - mutualisé.

Le calcul des montants collectés à l'aide de la taxe sur la masse salariale $\tau$ suppose de connaître l'état dans lequel se trouve chaque emploi suivant que le salaire versé a déjà été renégocié ou non, ainsi que la masse d'emplois qui se trouvent dans le même état. Parmi les emplois existants dans un secteur donné, un nombre $n_{o_{i}}$ d'entre eux a débuté avec une productivité supérieure ou égale au seuil de création $\varepsilon_{c_{i}}$ et n'a pas encore été affecté par un choc de productivité entraînant la renégociation du salaire. On note également $n_{e_{i}}$ le nombre d'emplois de ce secteur ayant déjà subi un choc; ces emplois ont une productivité supérieure ou égale au seuil de destruction $\varepsilon_{d_{i}}$. Le nombre respectif de ces emplois est régi par les équations suivantes :

$$
\dot{n}_{o_{i}}=\mu_{i} U_{i}-\left(\delta+\lambda_{i}\right) n_{o_{i}} \text { et } \dot{n}_{e_{i}}=\lambda_{i}\left(1-G_{i}\left(\varepsilon_{d_{i}}\right)\right) n_{o_{i}}-\left(\delta+\lambda_{i} G_{i}\left(\varepsilon_{d_{i}}\right)\right) n_{e_{i}}
$$

A chaque instant, $\mu_{i} U_{i}$ nouveaux emplois sont créés et s'ajoutent au stock des emplois dont le salaire n'a pas encore été renégocié. Dans le même temps, une fraction $\delta$ de ces emplois disparaît de manière exogène, tandis qu'une autre fraction de ces emplois est affectée par un choc qui survient au taux $\lambda_{i}$, entraînant la renégociation du salaire ou la destruction endogène de l'emploi. Ainsi, seule une fraction $1-G_{i}\left(\varepsilon_{d_{i}}\right)$ de ces $\lambda_{i} n_{o_{i}}$ emplois entre dans le stock des emplois dont le salaire a été renégocié. Le stock des emplois dont le salaire est renégocié diminue lui-même au taux $\delta+\lambda_{i} G_{i}\left(\varepsilon_{d_{i}}\right)$ en raison de la destruction du poste lorsque survient un choc trop important ou de façon exogène, du fait de la disparition du travailleur. A l'état stationnaire, on a :

$$
n_{o_{i}}=\frac{\mu_{i}}{\delta+\lambda_{i}} U_{i} \text { et } n_{e_{i}}=\frac{\lambda_{i}\left(1-G_{i}\left(\varepsilon_{d_{i}}\right)\right)}{\delta+\lambda_{i} G_{i}\left(\varepsilon_{d_{i}}\right)} n_{o_{i}}
$$

La contrainte de budget équilibrée s'écrit :

$$
\sum_{i} b_{i} U_{i}=\sum_{i} \tau\left[n_{o_{i}} \bar{w}_{o_{i}}+n_{e_{i}} \bar{w}_{e_{i}}\right]+\sum_{i} q_{i} E_{i} \tau_{e_{i}}
$$

où $\bar{w}_{o_{i}}=\int_{\varepsilon_{c_{i}}}^{\varepsilon_{u}} w_{o_{i}}(\varepsilon) \frac{d G_{i}(\varepsilon)}{1-G_{i}\left(\varepsilon_{c_{i}}\right)}$ et $\bar{w}_{e_{i}}=\int_{\varepsilon_{d_{i}}}^{\varepsilon_{u}} w_{e_{i}}(\varepsilon) \frac{d G_{i}(\varepsilon)}{1-G_{i}\left(\varepsilon_{d_{i}}\right)}$ représentent les salaires moyens respectivement parmi les emplois dont le salaire n'a pas encore été renégocié et parmi les emplois dont le salaire a déjà été renégocié. Le membre de gauche de (20) représente la masse totale des dépenses au titre du système d'assurance chômage, tandis que le membre de droite correspond à l'ensemble des ressources, qui se compose de deux éléments : le premier représente la part du financement assurée directement par des taxes sur les entreprises prélevées au taux $\tau$ sur la masse salariale totale $\sum_{i}\left[n_{o_{i}} \bar{w}_{o_{i}}+n_{e_{i}} \bar{w}_{e_{i}}\right]$, tandis que le second terme, $\sum_{i} q_{i} E_{i} \tau_{e_{i}}$, représente la part du système d'assurance chômage qui est 
financée par le système d'experience rating. Ce terme est relié au risque de destruction, de sorte que plus les licenciements sont fréquents, plus la contribution des firmes au financement de l'assurance chômage est importante. A partir de l'équation (20), nous obtenons directement le montant du taux de taxe uniforme $\tau$ en fonction de la taxe sur les licenciements $\tau_{e_{i}}$. Il vient:

$$
\tau=\frac{\sum_{i} b_{i} U_{i}-\sum_{i} q_{i} E_{i} \tau_{e_{i}}}{\sum_{i}\left[n_{o_{i}} \bar{w}_{o_{i}}+n_{e_{i}} \bar{w}_{e_{i}}\right]}
$$

Toute chose égale par ailleurs, la taxe sur la masse salariale, $\tau$, est une fonction décroissante de la taxe sur les licenciements, $\tau_{e_{i}}$. En effet, plus cette taxe sera importante ou alternativement plus le degré d'experience rating sera élevé, moins la part mutualisée du financement de l'assurance chômage sera grande.

Il convient en outre de remarquer que le financement de l'assurance chômage peut induire un phénomène de taxation/subventionnement entre les deux segments du marché du travail. Le montant de la subvention implicitement perçue par le segment $i$ (supportée pour un montant équivalent par l'autre secteur) peut ainsi s'écrire comme la différence entre le montant des subsides de l'assurance chômage reçus par les individus appartenant à ce segment du marché et les contributions versées. Il vient :

$$
T_{i}=b_{i} U_{i}-q_{i} E_{i} \tau_{e_{i}}-\tau\left[n_{o_{i}} \bar{w}_{o_{i}}+n_{e_{i}} \bar{w}_{e_{i}}\right]
$$

La taxe endogène sur les licenciements supportée par l'entreprise est corrélée au coût fiscal espéré, $C_{i}$, d'un chômeur de qualification $s_{i}$ pour la caisse d'assurance chômage. Ce coût vérifie :

$$
r C_{i}=b_{i}+\mu_{i}\left[0-C_{i}\right]
$$

Tout travailleur perçoit un subside de l'assurance chômage $b_{i}$ indexé sur sa qualification et sort du chômage au taux $\mu_{i}$, auquel cas le coût supporté par l'assurance chômage devient nul. En notant, $e$, l'indice de modulation des cotisations patronales à l'assurance chômage, la taxe sur les licenciements vérifie simplement $\tau_{e_{i}}=e C_{i}$. Par suite, il vient :

$$
\tau_{e_{i}}=\frac{e b_{i}}{r+\theta_{i} m_{i}\left(\theta_{i}\right)\left[1-G\left(\varepsilon_{c_{i}}\right)\right]}=\frac{e b_{i}}{r+\mu_{i}}
$$

Cette taxe est une fonction croissante de l'indice de modulation, e. En d'autres termes, quelque soit le niveau de qualification, une hausse de $e$ diminue la part mutualisée du coût d'un chômeur à la caisse d'assurance chômage et renchérit donc le coût supporté par la firme. La taxe $\tau_{e_{i}}$ est également liée au niveau de qualification $s_{i}$ pour deux raisons : tout d'abord, les subsides de l'assurance chômage sont généralement indexés sur les gains passés des travailleurs, et donc in fine sur leurs qualifications. Ensuite, des travailleurs de niveau de qualification différents sont en moyenne sujets à des épisodes de chômage dont la longueur varie avec la qualification. Ces deux éléments impliquent que le coût induit pour 
l'assurance chômage par une décision de licenciement est fonction de la qualification du travailleur, et qu'en conséquence, les coûts de séparation supportés par les firmes doivent internaliser (au moins en partie) ces coûts différents. La taxe sur les licenciements, $\tau_{e_{i}}$, est donc contingente au niveau de qualification des agents.

\section{Equilibre du marché du travail}

Nous présentons dans cette section les équations définissant les seuils d'embauche $\varepsilon_{c_{i}}$ de licenciement $\varepsilon_{d_{i}}$ et leurs propriétés lorsque la tension sur chacun des marchés $\theta_{i}$ et les taxes $\tau$ et $\tau_{e_{i}}$ sont données. Connaissant les propriétés de ces deux seuils, nous procédons alors à l'étude de la tension sur chaque marché $\theta_{i}$ à taxation donnée. Nous définissons finalement l'équilibre à taxation endogène; les effets d'équilibre général liés aux variations endogènes de la taxation sont étudiés dans la section 6 .

\subsection{Salaires}

A partir des valeurs (5) à (9), des règles de négociation salariale données en (3), et en imposant la libre entrée sur les marchés d'appariement, nous obtenons ${ }^{12}$ :

$$
\begin{gathered}
w_{o_{i}}(\varepsilon)=\frac{\beta_{i}}{(1+\tau)}\left[\varepsilon+s_{i}-\lambda_{i}\left(f_{i}+\tau_{e_{i}}\right)\right]+(1-\beta) r V_{u_{i}} \\
w_{e_{i}}(\varepsilon)=\frac{\beta_{i}}{(1+\tau)}\left[\varepsilon+s_{i}+r\left(f_{i}+\tau_{e_{i}}\right)\right]+(1-\beta) r V_{u_{i}}
\end{gathered}
$$

Ces expressions possèdent des propriétés assez standards. Le salaire est une moyenne pondérée de la productivité de l'emploi et de l'utilité de réserve du travailleur en raison du partage de rente qui s'opère dans la négociation. Il croît avec la qualification $s_{i}$ du travailleur qui affecte la taille du surplus à se partager ainsi qu'avec la tension du marché du travail $\theta_{i}$ qui affecte les opportunités externes du travailleur. Il décroît avec le taux de taxe $\tau$ qui réduit la taille du surplus à partager : la négociation implique ainsi que le travailleur supporte en partie cette taxe même si elle est payée par l'entreprise. On remarque aussi que $w_{o_{i}}(\varepsilon)-w_{e_{i}}(\varepsilon)=-\left(r+\lambda_{i}\right)\left(f_{i}+\tau_{e_{i}}\right)$. Cette différence s'explique par le fait que la négociation salariale implique un transfert de ceux qui ne sont pas encore en emploi vers ceux qui sont déjà employés. L'employeur sait qu'une fois embauché le travailleur utilisera la protection de l'emploi pour accroître son salaire - d'où le terme $r\left(f_{i}+\tau_{e_{i}}\right)$ dans l'expression (26) - et utilise le fait que ceux qui ne sont pas encore embauchés ne peuvent se prévaloir des effets de la protection de l'emploi. Ces derniers sont prêts à accepter une baisse de leur salaire pour être embauchés, d'où le terme $-\lambda_{i}\left(f_{i}+\tau_{e_{i}}\right)$ dans l'expression du salaire (25).

12. Le détail des calculs est donné dans l'Annexe 5.1. 


\subsection{Seuil de création et seuil de destruction des emplois}

Le seuil de création d'emplois est déterminé à l'aide de la règle de surplus nul donnée par (10) et satisfait pour chaque niveau de qualification ${ }^{13}$ :

$$
0=\varepsilon_{c_{i}}+s_{i}-\lambda_{i}\left(f_{i}+\tau_{e_{i}}\right)+\frac{\lambda_{i}}{r+\lambda_{i}} \int_{\varepsilon_{d_{i}}}^{\varepsilon_{u_{i}}}\left(\zeta-\varepsilon_{d_{i}}\right) d G_{i}(\zeta)-r V_{u_{i}}(1+\tau)
$$

Le seuil de destruction des emplois est quant à lui déterminé par la règle de surplus nul (11) et vérifie pour chaque niveau de qualification :

$$
0=\varepsilon_{d_{i}}+s_{i}+r\left(f_{i}+\tau_{e_{i}}\right)+\frac{\lambda_{i}}{r+\lambda_{i}} \int_{\varepsilon_{d_{i}}}^{\varepsilon_{u_{i}}}\left(\zeta-\varepsilon_{d_{i}}\right) d G_{i}(\zeta)-r V_{u_{i}}(1+\tau)
$$

En confrontant ces deux expressions, il vient :

$$
\varepsilon_{c_{i}}=\varepsilon_{d_{i}}+\left(r+\lambda_{i}\right)\left(f_{i}+\tau_{e_{i}}\right)
$$

Cette relation montre que le seuil de création des emplois est plus élevé que le seuil de destruction en raison des coûts de séparation $f_{i}+\tau_{e_{i}}$. L'interprétation de cette propriété est simple : les employeurs tiennent compte au moment de l'embauche du fait qu'ils auront à subir les coûts de licenciement dans l'éventualité où le poste serait détruit; ils se montrent alors plus exigeants sur la productivité nécessaire à une embauche en anticipant les coûts de séparation qui résulteraient d'une destruction.

En annexe, nous montrons que :

$$
r V_{u_{i}}=b_{i}+\frac{\beta_{i} \theta_{i} m_{i}\left(\theta_{i}\right)}{(1+\tau)\left(r+\lambda_{i}\right)} \int_{\varepsilon_{c_{i}}}^{\varepsilon_{u_{i}}}\left(\zeta-\varepsilon_{c_{i}}\right) d G_{i}(\zeta)
$$

En faisant usage de cette expression et de la relation (29) dans (27) et (28), on peut alors en déduire les propriétés de statique comparative des seuils $\varepsilon_{c_{i}}$ et $\varepsilon_{d_{i}}$, résumées dans le tableau suivant :

$$
\begin{array}{cccccccc} 
& s_{i} & \theta_{i} & f_{i} & \tau_{e_{i}} & b_{i} & \tau & \beta_{i} \\
\varepsilon_{c_{i}} & - & + & + & + & + & + & + \\
\varepsilon_{d_{i}} & - & + & - & - & + & + & +
\end{array}
$$

STATIQUE COMPARATIVE DES SEUILS $\varepsilon_{c_{i}}$ ET $\varepsilon_{d_{i}}$

Les expressions (27) et (28) montrent ainsi que les seuils d'embauche et de licenciement diminuent avec la productivité spécifique au travailleur $s_{i}{ }^{14}$. Plus le surplus attendu de

13. Le détail des calculs pour déterminer ces productivités de réservation est présenté dans l'Annexe 5.1 .

14. Ceci est du moins le cas dès lors que les paramètres du modèle ne sont pas trop fortement indexés sur les compétences des travailleurs. Nous considérerons uniquement des configurations paramétriques qui garantissent cette propriété. 
toute relation d'emploi est important, plus firmes et travailleurs sont prêts à accepter une productivité spécifique initiale $\varepsilon_{c_{i}}$ faible; ils sont également d'autant plus réticents à se séparer en cas de choc défavorable qu'ils peuvent attendre un gain de la relation d'emploi qui sera élevé. Les seuils $\varepsilon_{c_{i}}$ et $\varepsilon_{d_{i}}$ sont également croissants avec la tension $\theta_{i}$ : lorsque la tension s'élève, le surplus associé à tout emploi diminue, de sorte que les parties se montrent donc plus exigeantes quant au niveau des productivités de réserve requises pour qu'un emploi puisse voir le jour ou pour qu'une relation d'emploi puisse continuer. Les coûts de séparation $f_{i}$ et $\tau_{e_{i}}$ qui ont pour objet de réduire les destructions d'emploi ont également pour effet d'élever le seuil de création $\varepsilon_{c_{i}}$ : les employeurs tiennent compte lors de la création d'un emploi du fait qu'ils devront supporter ces coûts dans l'éventualité où l'emploi serait détruit. Cet effet réduit le surplus attendu de toute formation d'un emploi, de sorte qu'il faut un niveau de productivité spécifique à l'emploi d'autant plus élevé que ces coûts de séparation sont importants pour que la relation d'emploi puisse débuter de manière rentable. Enfin, les seuils $\varepsilon_{c_{i}}$ et $\varepsilon_{d_{i}}$ augmentent tous deux avec le niveau des allocations chômage $b_{i}$, et le taux de taxe $\tau$, qui ont pour effet de réduire le surplus associé à tout emploi (nouvellement créé ou existant), ce qui limite les créations d'emplois et encouragent les destructions.

\subsection{Equilibre à taxation donnée}

Nous endogénéisons à présent la demande de travail à partir de la condition de libre entrée (12) qui prévaut sur chaque marché. La tension $\theta_{i}$ vérifie l'expression suivante :

$$
\frac{c_{i}}{m_{i}\left(\theta_{i}\right)}=\frac{1-\beta_{i}}{r+\lambda_{i}} \int_{\varepsilon_{c_{i}}}^{\varepsilon_{u_{i}}}\left(\zeta-\varepsilon_{c_{i}}\right) d G_{i}(\zeta)
$$

La libre entrée implique que de nouvelles entreprises entrent sur le marché jusqu'à égaliser la valeur attendue des coûts de recherche $\frac{c_{i}}{m_{i}\left(\theta_{i}\right)}$ à la valeur attendue d'un emploi $\left(1-\beta_{i}\right)$ $\int_{\varepsilon_{c_{i}}}^{\varepsilon_{u}} \frac{\zeta-\varepsilon_{c_{i}}}{r+\lambda_{i}} d G_{i}(\zeta)$. Combinée avec l'expression du seuil d'embauche $\varepsilon_{c_{i}}$, et en tenant compte de la relation (29) entre les seuils d'embauche et de licenciement, ceci définit l'équilibre du marché du travail à taxation donnée comme une paire $\left(\varepsilon_{c_{i}}, \theta_{i}\right)$ vérifiant l'équation de libre entrée (31) et la relation :

$$
\begin{aligned}
0= & \varepsilon_{c_{i}}+s_{i}-\lambda_{i}\left(f_{i}+\tau_{e_{i}}\right)-b_{i}(1+\tau)-\frac{\beta_{i} \theta_{i} m_{i}\left(\theta_{i}\right)}{r+\lambda_{i}} \int_{\varepsilon_{c_{i}}}^{\varepsilon_{u_{i}}}\left(\zeta-\varepsilon_{c_{i}}\right) d G_{i}(\zeta) \\
& +\frac{\lambda_{i}}{r+\lambda_{i}} \int_{\varepsilon_{c_{i}}-\left(r+\lambda_{i}\right)\left(f_{i}+\tau_{e_{i}}\right)}^{\varepsilon_{u_{i}}}\left(\zeta-\varepsilon_{c_{i}}+\left(r+\lambda_{i}\right)\left(f_{i}+\tau_{e_{i}}\right)\right) d G_{i}(\zeta)
\end{aligned}
$$

Connaissant les valeurs de la tension et du seuil d'embauche définit par ces expressions, il est possible d'en déduire le seuil de destruction à l'aide de (29). Connaissant la valeur d'équilibre de la tension, la décomposition du ratio $\theta_{i}$ entre nombre de chômeurs et emplois 
vacants découle alors de (17). Au regard des deux expressions ci-dessus, il est clair que l'existence d'une solution n'est pas toujours assurée : pour des valeurs suffisamment faibles de $s_{i}$ et/ou des rigidités institutionnelles suffisamment importantes, il est possible que l'on ait $\varepsilon_{c_{i}}>\varepsilon_{u}$, de sorte que $S_{o_{i}}\left(\varepsilon_{u}\right)<0$, ce qui implique également $\theta_{i}=0$ d'après (31). Les travailleurs qui se retrouvent dans cette configuration sont inemployables et choisissent rationnellement l'inactivité. On peut ainsi définir $\underline{s}$ comme le niveau de qualification minimal requis pour être employable. A l'aide de (32), ce seuil vérifie :

$$
\underline{s}=-\varepsilon_{u}+b_{i}(1+\tau)+\frac{\lambda_{i}}{r+\lambda_{i}} \int_{\varepsilon_{u_{i}}-\left(r+\lambda_{i}\right)\left(f_{i}+\tau_{e_{i}}\right)}^{\varepsilon_{u_{i}}} G_{i}(\zeta) d \zeta
$$

Nous pouvons dès lors établir les propriétés suivantes :

$$
\begin{aligned}
& \begin{array}{lllll}
f_{i} & \tau_{e_{i}} & b_{i} & \tau & \beta_{i}
\end{array} \\
& \underline{s}+++++
\end{aligned}
$$

\section{PROPRIÉTÉS DU SEUIL D'EMPLOYABILITÉ $\underline{s}$}

Nous nous concentrons à présent sur les configurations telles que $s_{2}>\underline{s}$. Dans la mesure où $s_{1}>s_{2}$, ceci suffit à garantir l'existence de l'équilibre sur chaque marché. La solution au système formé des équations (31) et (32) est unique dans la mesure où la première expression définit la tension $\theta_{i}$ comme une fonction décroissante du seuil $\varepsilon_{c_{i}}$, tandis que la seconde définit le seuil $\varepsilon_{c_{i}}$ comme une fonction croissante de la tension $\theta_{i}$. Formellement, il vient :

$$
\begin{gathered}
\left.\frac{\partial \theta_{i}}{\partial \varepsilon_{c_{i}}}\right|_{(31)}=\frac{\left(1-\beta_{i}\right)\left(1-G_{i}\left(\varepsilon_{c_{i}}\right)\right) m_{i}\left(\theta_{i}\right)^{2}}{c_{i} m_{i}^{\prime}\left(\theta_{i}\right)}<0 \\
\left.\frac{\partial \theta_{i}}{\partial \varepsilon_{c_{i}}}\right|_{(32)}=\frac{r+\beta_{i} \theta_{i} m_{i}\left(\theta_{i}\right)\left(1-G_{i}\left(\varepsilon_{c_{i}}\right)\right)+\lambda_{i} G_{i}\left(\varepsilon_{d_{i}}\right)}{\beta_{i} m_{i}\left(\theta_{i}\right)\left[1-\eta_{i}\left(\theta_{i}\right)\right] \int_{\varepsilon_{c_{i}}}^{\varepsilon_{u}}\left(\zeta-\varepsilon_{c_{i}}\right) d G_{i}(\zeta)}>0
\end{gathered}
$$

avec $m^{\prime}\left(\theta_{i}\right)<0$ et $\eta_{i}\left(\theta_{i}\right)$, l'élasticité de la fonction d'appariement par rapport au chômage, comprise entre $[0 ; 1]$.

On peut alors établir les propriétés de statique comparative de la tension $\theta_{i}$ qui prévaut sur chaque marché :

$$
\begin{array}{ccccccc} 
& s_{i} & f_{i} & \tau_{e_{i}} & b_{i} & \tau & \beta_{i} \\
\theta_{i} & + & - & - & - & - & -
\end{array}
$$

PropriÉtÉS DE LA TENSION $\theta_{i}$

La tension $\theta_{i}$ est ainsi une fonction croissante de la productivité individuelle $s_{i}$, et une fonction décroissante du niveau des allocations chômage $b_{i}$, de la taxe $\tau$, ou encore des coûts de séparation $f_{i}$ et $\tau_{e_{i}}$. En effet, le surplus attendu de toute rencontre augmente avec $s_{i}$ alors qu'il diminue avec $b_{i}, \tau, f_{i}$ et $\tau_{e_{i}}$, d'où l'évolution de la tension avec ces paramètres. 
Ainsi, lorsque l'on raisonne à fiscalité donnée, une variation des coûts de licenciement $f_{i}$ ou de la taxe d'experience rating $\tau_{e_{i}}$ a des effets strictement identiques dans la mesure où 15

$$
\frac{d \varepsilon_{c_{i}}}{d f_{i}}=\frac{d \varepsilon_{c_{i}}}{d \tau_{e_{i}}} ; \frac{d \varepsilon_{d_{i}}}{d f_{i}}=\frac{d \varepsilon_{d_{i}}}{d \tau_{e_{i}}} ; \frac{d \theta_{i}}{d f_{i}}=\frac{d \theta_{i}}{d \tau_{e_{i}}}
$$

\subsection{Equilibre à taxation endogène}

La prise en compte de la contrainte budgétaire implique désormais une interdépendance entre les marchés qui n'existait pas à taxation donnée. Il n'est donc plus possible d'étudier l'équilibre d'un marché indépendamment de ce qui se passe sur l'autre. De surcroît, l'endogénéisation des taxes données par (21) et (24) implique qu'il faut tenir compte des équations de salaires (25) et (26) ainsi que des équations de flux données en (17) et (19).

Definition : Un équilibre à taxation endogène est ainsi composé des définitions des seuils de création $\varepsilon_{c_{1}}^{*}$ et $\varepsilon_{c_{2}}^{*}$, de destruction $\varepsilon_{d_{1}}^{*}$ et $\varepsilon_{d_{2}}^{*}$ établis en (32) et (29), de la tension sur chaque marché $\theta_{1}^{*}$ et $\theta_{2}^{*}$ définie en (31), ainsi que des taxes $\tau^{*}, \tau_{e_{1}}^{*}$ et $\tau_{e_{2}}^{*}$ données par (21) et (24), des équations de salaire définies en (25) et (26) ainsi que des équations d'équilibre des flux définissant $U_{1}^{*}, U_{2}^{*}, n_{o_{1}}^{*}, n_{o_{2}}^{*}, n_{e_{1}}^{*}$ et $n_{e_{2}}^{*}$ données en (17) et (19).

La prise en compte de la contrainte de budget rend difficile l'étude du modèle de manière analytique. Deux points peuvent cependant être remarqués : (i) tout d'abord, un changement spécifique à un marché est ici susceptible de se répercuter sur l'autre via les modifications de la taxation. En effet, considérons une variation de coûts de licenciement sur le marché $\mathrm{j}, f_{j}$. Si l'on se trouve dans une configuration où $\frac{d \tau}{d f_{j}} \geq 0$, on en déduit que :

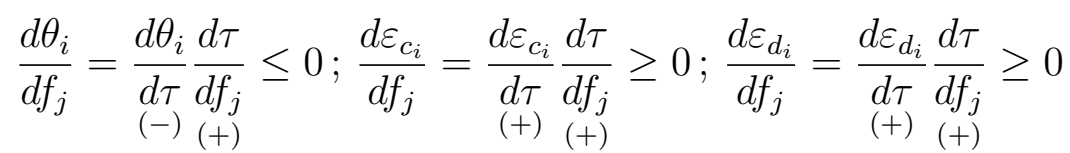

pour $i=1,2$ et $j \neq i$. Et par suite :

$$
\frac{d u_{i}}{d f_{j}}=\frac{d u_{i}}{d \theta_{i}} \frac{d \theta_{i}}{d f_{j}}+\frac{d u_{i}}{d \varepsilon_{c_{i}}} \frac{d \varepsilon_{c_{i}}}{d f_{j}}+\frac{d u_{i}}{d \varepsilon_{d_{i}}} \frac{d \varepsilon_{d_{i}}}{d f_{j}} \geq 0
$$

La hausse du chômage sur un marché peut entraîner une hausse du taux de taxe $\tau$. Dans la mesure où toute variation de ce taux de taxe affecte positivement les productivités de réserve $\varepsilon_{c_{i}}$ et $\varepsilon_{d_{i}}$ et négativement la tension $\theta_{i}$ sur l'autre marché, toute variation à la hausse de ce taux de taxe $\tau$ peut entraîner un accroissement du chômage sur cet autre marché.

15. L'Annexe 5.2 montre en effet que les expressions des dérivées à l'équilibre par rapport à $f_{i}$ et à $\tau_{e_{i}}$ sont identiques. 
Un argument similaire permet également d'établir que pour tout changement d'un paramètre $x_{j}$ spécifique au marché $j$, tel que $\frac{d \tau}{d x_{j}} \leq 0$, a les conséquences suivantes au sein du secteur $j$ :

$$
\frac{d \theta_{i}}{d x_{j}}=\frac{d \theta_{i}}{d \tau} \frac{d \tau}{d x_{j}} \geq 0 ; \frac{d \varepsilon_{c_{i}}}{d x_{j}}=\frac{d \varepsilon_{c_{i}}}{d \tau} \frac{d \tau}{d x_{j}} \leq 0 ; \frac{d \varepsilon_{d_{i}}}{d x_{j}}=\frac{d \varepsilon_{d_{i}}}{d \tau} \frac{d \tau}{d x_{j}} \leq 0
$$

pour $i=1,2$ et $j \neq i$. Et par suite :

$$
\frac{d u_{i}}{d x_{j}}=\frac{d u_{i}}{d \theta_{i}} \frac{d \theta_{i}}{d x_{j}}+\frac{d u_{i}}{d \varepsilon_{c_{i}}} \frac{d \varepsilon_{c_{i}}}{d x_{j}}+\frac{d u_{i}}{d \varepsilon_{d_{i}}} \frac{d \varepsilon_{d_{i}}}{d x_{j}} \leq 0
$$

Ceci sera le cas par exemple quand la productivité $s_{j}$ spécifique aux travailleurs du secteur $j$ augmente. Cet effet est illustré numériquement en section 6 .

(ii) Ensuite, l'interdépendance entre les deux marchés peut éventuellement déboucher sur une multiplicité d'équilibres. Ce résultat est habituel dans les modèles d'appariement avec taxation endogène, où toute création d'emploi supplémentaire par une firme est susceptible d'entraîner une baisse de la taxation, alors qu'en retour la baisse de la taxation induit une augmentation de la rentabilité attendue des emplois, ce qui favorise l'emploi. Ce mécanisme peut ici se propager de manière intersectorielle : toute baisse du chômage dans un secteur entraîne une baisse de la taxation qui bénéficie également à l'autre secteur, et y favorise également l'emploi, entraînant une nouvelle baisse de la taxation, etc. Dans une telle éventualité, il est raisonnable de supposer que le gouvernement a la possibilité de choisir le taux de taxe qui assure le plus faible taux de chômage et peut ainsi éviter tout équilibre pathologique.

L'objet des développements qui suivent est d'étudier les effets de l'experience rating et des mesures traditionnelles de la LPE qui peuvent être différenciés dès lors que l'on prend en compte l'endogénéité de la taxation et la contrainte budgétaire. Dans cette optique, nous procédons à une série d'exercices numériques afin de souligner les contrastes entre les différentes modalités de protection de l'emploi. Avant de passer à cette étude réalisée en section 6, nous nous intéressons dans la section qui suit aux effets de la LPE sur la structure par qualification de la population et la productivité moyenne des emplois.

\section{$5 \quad$ Les effets de la LPE sur la productivité des emplois et les décisions d'éducation}

De par son impact sur les créations et destructions d'emplois, la LPE affecte la structure par qualification de la population, ce qui implique que ses effets sur l'emploi et la productivité moyenne des emplois peuvent différer à court terme lorsque la structure par 
qualification de la population est donnée, et à plus long terme quand celle-ci est endogène. Cette section cherche à mettre en évidence ces effets qui seront quantifiés en section 6 .

\subsection{Les effets de la LPE sur la productivité des emplois}

Pour mettre en évidence les différents canaux par lesquels la LPE est susceptible d'affecter la productivité moyenne de l'économie, commençons par considérer l'expression de la productivité moyenne des emplois au sein du secteur $i^{16}$ :

$$
\bar{y}_{i}=s_{i}+\frac{n_{o_{i}}}{n_{o_{i}}+n_{e_{i}}} \int_{\varepsilon_{c_{i}}}^{\varepsilon_{u_{i}}} x \frac{d G_{i}(x)}{1-G_{i}\left(\varepsilon_{c_{i}}\right)}+\frac{n_{e_{i}}}{n_{o_{i}}+n_{e_{i}}} \int_{\varepsilon_{d_{i}}}^{\varepsilon_{u_{i}}} x \frac{d G_{i}(x)}{1-G_{i}\left(\varepsilon_{d_{i}}\right)}
$$

où $\frac{n_{o_{i}}}{n_{o_{i}}+n_{e_{i}}}$ représente la part d'emplois nouvellement créés dans l'emploi du secteur $i$, égale à $\frac{\delta+\lambda_{i} G_{i}\left(\varepsilon_{d_{i}}\right)}{\delta+\lambda_{i}}$ d'après $(19)$.

La productivité moyenne des emplois de chaque secteur dépend ainsi de la productivités des travailleurs $s_{i}$, et de la valeur moyenne prise par la composante aléatoire de la productivité parmi les emplois nouvellement créés ou existant. Elle est affectée par le LPE qui modifie les seuils $\varepsilon_{c_{i}}$ et $\varepsilon_{d_{i}}$, ce qui donne lieu à des effets contradictoires : (i) en augmentant le seuil de création $\varepsilon_{c_{i}}$, la LPE contribue à augmenter la productivité moyenne des emplois nouvellement créés; (ii) en réduisant le seuil $\varepsilon_{d_{i}}$, la LPE contribue à maintenir en place des emplois dont la productivité est faible, ce qui a un impact négatif sur la productivité moyenne de chaque secteur; (iii) en abaissant le seuil $\varepsilon_{d_{i}}$, la LPE contribue à accroître la proportion d'emplois existants par rapports aux emplois nouvellement créés, et dans la mesure où la productivité des premiers est moins élevée que celle des seconds, elle réduit par ce biais la productivité moyenne $\bar{y}_{i}$.

La productivité moyenne de l'économie est alors une moyenne pondérée des productivités des emplois de chaque secteur, égale à :

$$
\bar{y}=\frac{\sum_{i}\left(1-u_{i}\right) P_{i} \bar{y}_{i}}{\sum_{i}\left(1-u_{i}\right) P_{i}}
$$

où $u_{i}=\frac{\delta+\lambda_{i} G_{i}\left(\varepsilon_{d_{i}}\right)}{\delta+\lambda_{i} G_{i}\left(\varepsilon_{d_{i}}\right)+\theta_{i} m_{i}\left(\theta_{i}\right)\left(1-G_{i}\left(\varepsilon_{c_{i}}\right)\right)}$ d'après $(17)$. Cette expression suggère que tout changement de la LPE affecte la productivité moyenne de l'économie à travers trois canaux : (i) tout d'abord, de par les changements de la productivité moyenne $\bar{y}_{i}$ au sein de chaque secteur (ii) ensuite, en modifiant les taux d'emplois $\left(1-u_{i}\right)$ de chaque secteur (iii) et enfin, à plus long terme, lorsque la proportion $P_{i}$ est endogène, à travers les changements induits dans les incitations à s'éduquer.

Notons que si ces effets sont a priori ambigus, le point intéressant est ici qu'ils ont une chance d'être positifs, notamment du fait de l'impact de la LPE sur les incitations à s'éduquer. Ces choix éducatifs peuvent être modélisés comme suit.

16. Le détail des calculs est reporté dans l'Annexe 5.3. 


\subsection{Les effets de la LPE sur les décisions d'éducation}

La proportion $P_{i}$ peut être endogénéisée en considérant que celle-ci résulte d'un investissement en formation initiale qui prend la forme d'un choix binaire : s'éduquer/ne pas s'éduquer ${ }^{17}$. La population est hétérogène par rapport au coût d'éducation noté $C$, distribuée selon un fonction de répartition notée $\Phi$. Ainsi, les individus s'éduquent à condition que

$$
r\left(V_{u_{1}}-V_{u_{2}}\right) \geq C
$$

Il existe ainsi un individu limite dans la population, qui étant donné le coût d'éducation dont il est doté, et étant donné le rendement lié au fait d'acquérir un diplôme, est indifférent entre se qualifier ou non. Nous noterons $\widehat{C}$ la caractéristique de cet individu, telle que

$$
r\left(V_{u_{1}}-V_{u_{2}}\right)=\widehat{C}
$$

où $r V_{u_{i}}=b_{i}+\frac{\beta_{i} \theta_{i} m_{i}\left(\theta_{i}\right)}{(1+\tau)\left(r+\lambda_{i}\right)} \int_{\varepsilon_{c_{i}}}^{\varepsilon_{u_{i}}}\left(\zeta-\varepsilon_{c_{i}}\right) d G_{i}(\zeta)$ d'après (30). La proportion $P_{1}$ d'individus qualifiés est ainsi égale à

$$
P_{1}=\Phi(\widehat{C})
$$

Tant que l'on raisonne à taxation donnée, le modèle conserve une structure récursive simple : connaissant les valeurs d'équilibre de la tension et des seuils de destruction sur chaque marché, on en déduit les valeurs de $r V_{u_{1}}$ et $r V_{u_{2}}$, et par suite, celle du rendement de l'éducation et de la proportion d'individus qualifiés qui en découlent.

A taxation donnée, la structure par qualification de la population évolue ainsi comme suit en fonction des paramètres de politique économique :

$$
\begin{array}{cccccc}
f_{1} & f_{2} & \tau_{e_{1}} & \tau_{e_{2}} & \tau \\
P_{1} & - & + & - & + & ? \\
\text { POLITIQUE ÉCONOMIQUE ET } & \text { DÉCISIONS D'ÉDUCATION }
\end{array}
$$

Ceci implique que la productivité moyenne de l'économie est susceptible d'augmenter avec les paramètres $f_{2}$ et $\tau_{e_{2}}$ et de diminuer avec les paramètres $f_{1}$ et $\tau_{e_{1}}$. Le paramètre $\tau$ qui affecte dans le même sens l'utilité attendue de la recherche d'emploi sur les marchés qualifiés et non qualifiés exerce ainsi un effet ambigu sur les incitations à s'éduquer. Au total, ceci permet d'établir qu'à taxation donnée, à l'instar de (34) on obtient:

$$
\frac{d P_{1}}{d f_{1}}=\frac{d P_{1}}{d \tau_{e_{1}}} ; \frac{d P_{1}}{d f_{2}}=\frac{d P_{1}}{d \tau_{e_{2}}}
$$

17. Un grand nombre de contributions analysent les choix d'éducation dans des modèles duaux (e.g. Saint-Paul, 1996). En revanche, un nombre relativement restreint s'intéressent au lien entre éducation et protection de l'emploi. Charlot (2006) considère un investissement en formation initial qui prend la forme d'un effort d'éducation, et montre que le problème de hold up auquel est soumis l'investissement éducatif peut justifier le recours à la LPE. Wasmer (2006) envisage les effets de la protection de l'emploi sur la formation acquise pendant l'emploi, qui peut être de nature générale ou spécialisée. 
Ce qui implique aussi, à l'aide de (34) et de l'expression de la productivité moyenne $\bar{y}$ :

$$
\frac{d \bar{y}}{d f_{i}}=\frac{d \bar{y}}{d \tau_{e_{i}}}
$$

Il en va différemment à taxation endogène comme le montrera la section suivante ${ }^{18}$.

\section{Politique économique}

Notre but est ici de de mettre en lumière les éléments suivants : (i) En l'absence d'experience rating, le financement de l'assurance chômage induit un phénomène de subventionnement entre le secteur qualifié et le secteur non qualifié. Dans la mesure où dans la réalité, le ratio de remplacement est plus généreux pour les non qualifiés que pour les qualifiés, que les premiers sont davantage au chômage que les seconds, et que le financement de l'assurance chômage s'effectue initialement sur la base d'un taux de taxe uniforme, le subventionnement s'effectue vraisemblablement au profit des non qualifiés; (ii) l'introduction d'une taxe sur les licenciements indexée sur la durée espérée des épisodes de chômage devrait induire une réduction de la subvention entre les différents segments du marché du travail ; (iii) l'introduction de la taxe sur les licenciements est susceptible d'accroître le niveau des rigidités, en particulier pour les travailleurs non qualifiés dont les durées de chômage sont plus élevées que pour les qualifiés. Cette taxe risque donc de rallonger encore davantage la durée de leurs épisodes de chômage; (iv) Enfin, l'introduction de la taxe d'experience rating s'accompagne d'une contrepartie fiscale ${ }^{19}$ d'autant plus importante que l'indice de modulation est élevé. Cette contrepartie fiscale se traduit alors par une baisse du taux de taxe uniforme qui affecte chaque segment du marché du travail.

Une évaluation plus précise s'avère donc indispensable pour évaluer l'impact global de cette réforme. Dans cette optique, la suite de cette section se concentre sur une série d'exercices numériques. Nous établissons une situation de référence, à partir de laquelle nous cherchons à mettre en évidence l'impact de la protection de l'emploi "traditionnelle" sur le niveau et la composition du chômage par qualification, ainsi que sur la productivité des emplois et les incitations à se qualifier. Nous nous intéressons ensuite aux effets associés à un système de modulation des cotisations patronales à l'assurance chômage.

\subsection{Une situation de référence}

Afin de fixer les idées, nous illustrons notre modèle sur un exemple numérique. La période de référence est l'année et le taux d'escompte effectif $r=\rho+\delta$ est égal à $5 \%$. Le taux de

18. L'étude de cet équilibre se ramène in fine essentiellement à celle d'un point fixe entre la proportion d'individus qualifiés $P_{1}^{*}$ et le taux de $\tau^{*}$.

19. Pour plus de détails voir sur ce point Cahuc et Malherbet (2004). 
natalité $\delta$ est fixé à $1,5 \%$. Nous considérons ici deux groupes de qualification, indexés par $i=1$ ou 2. A l'instar de Mortensen et Pissarides (2003), nous supposons que la fonction d'appariement est de type Cobb-Douglas et satisfait : $M_{i}\left(U_{i}, V_{i}\right)=k_{i} U_{i}^{\eta_{i}} V_{i}^{1-\eta_{i}}$ où $\eta_{i}$ est l'élasticité de la fonction d'appariement et $k_{i}$ est un paramètre d'efficacité spécifique à chaque marché. Le paramètre d'élasticité de la fonction d'appariement est fixé à $\eta_{1}=$ $\eta_{2}=0,5$ (Petrongolo et Pissarides, 2001) et nous choisissons de l'égaliser à la valeur du pouvoir de négociation des travailleurs, i.e. $\beta_{1}=\beta_{2}=0,5$, en accord avec la majeure partie de la littérature (e.g. Blanchard et Diamond, 1989, Millard et Mortensen, 1997). La valeur des paramètres de productivité $s_{1}$ et $s_{2}$ sont $s_{1}=1,571$ et $s_{2}=0,835$ en accord avec les estimations de Crépon, Deniau et Perez-Duarte (2002).

Les autres paramètres de politique économique sont choisis afin de fournir des valeurs pouvant correspondre au cas français. Les allocations chômage, $b_{i}$, sont indexées sur les salaires par niveau de qualification conformément à la législation en vigueur en France, où l'indemnisation du chômage est relativement plus généreuse pour les travailleurs à faible qualification que pour les autres. Le paramètre d'indexation pour les qualifiés vaut $\omega_{1}=0,574$ et pour les non qualifiés $\omega_{2}=0,75$ (UNEDIC, 2006). La rigueur de la LPE est modulée par le paramètre $\gamma$; lorsque ce paramètre vaut 1 , le coût des procédures administratives de licenciements représente 4 mois de salaire moyen (Kramarz et Michaud, 2004); lorsque ce paramètre vaut 2, le coût des procédures administratives de licenciements représente alors 8 mois de salaire moyen. Les valeurs de ces paramètres sont résumées dans le tableau qui suit:

\begin{tabular}{lccc}
\hline \hline Paramètres imposés & Notation & Qualifié & Non Qualifié \\
\hline \hline Elasticité de la fonction d'appariement & $\eta_{i}$ & 0,5 & 0,5 \\
Pouvoir de négociation & $\beta_{i}$ & 0,5 & 0,5 \\
Taux d'escompte & $r$ & 0,05 & 0,05 \\
Taux de natalité (mortalité) & $\delta$ & 0,015 & 0,015 \\
Paramètre de productivité spécifique au travailleur & $s_{i}$ & 1,571 & 0,835 \\
Indice de modulation & $e$ & 0 & 0 \\
Indice de rigueur de la LPE & $\gamma$ & 1 & 1 \\
Ratio de remplacement & $\omega_{i}$ & 0,574 & 0,75 \\
\hline \hline
\end{tabular}

La distribution des chocs de productivité tout comme la distribution des coûts d'éducation sont supposées uniformes, respectivement sur le support $\left[\varepsilon_{l_{i}}, 1\right]$ pour les chocs de productivité et sur $[0,1]$ pour les coûts d'éducation. De la sorte, il nous reste 8 paramètres $-k_{i}, \lambda_{i}$, $c_{i}$ et $\varepsilon_{l_{i}}$ - pouvant être fixés de manière à reproduire des valeurs correspondant à celles d'une économie comme la France. Les paramètres $k_{i}$ et $c_{i}$ jouant un rôle très similaire dans ce modèle, nous choisissons de fixer arbitrairement $c_{1}=0,6$ et $c_{2}=0,25,-$ le coût 
de postage d'un emploi représentant ainsi environ $1 / 3$ de la productivité $s_{i}$ du travailleur - tout en ajustant les paramètres d'efficacité de la fonction d'appariement sur chaque marché $k_{1}$ et $k_{2}$, ce qui laisse au final 6 paramètres libres; les contraintes auxquelles nous nous astreignons dans le choix de ces paramètres sont résumées dans le tableau suivant:

\begin{tabular}{ll}
\hline \hline Contraintes sur les paramètres libres & Sources \\
\hline \hline $\bar{q}=P_{1} q_{1}+\left(1-P_{1}\right) q_{2} \approx 10 \%$ & Duhautois (2002) \\
$\bar{\mu}^{-1}=\left(P_{1} \mu_{1}+\left(1-P_{1}\right) \mu_{2}\right)^{-1} \approx 1$ & OCDE, perspectives de l'emploi \\
$u_{1} \approx 6 \%$ et $u_{2} \approx 14 \%$ & OCDE, perspectives de l'emploi \\
$P_{1} \approx 60 \%$ & Manacorda et Petrongolo (1999) \\
$\tau \approx 6,4 \%$ & UNEDIC (2006) \\
\hline \hline
\end{tabular}

Nous cherchons à reproduire (1) un taux de destruction agrégé de l'ordre de 10\% (Duhautois, 2002) (2) une durée moyenne du chômage de l'ordre d'une année, en accord avec celle observée en France (OCDE, 2006), ainsi que des taux de chômage par qualification, qui devraient être de l'ordre de $6 \%$ pour les travailleurs qualifiés et $14 \%$ pour les non qualifiés, ce qui donne les contraintes (3) et (4). Enfin, on doit retrouver (5) une proportion de travailleurs qualifiés, $P$ environ égale à $60 \%$, en adéquation avec les chiffres fournis par Manacorda et Petrongolo (1999) et (6) un taux de taxe $\tau$ destiné à financer l'assurance chômage voisin de celui constaté, qui vaut $6,4 \%$ (UNEDIC, 2006).

De surcroît, parmi les configurations compatibles avec ces valeurs, nous en retenons une permettant de reproduire des écarts suffisamment importants dans les taux d'entrées et de sortie du chômage par qualification, conformément à ce que constate la majeure partie de la littérature empirique sur le sujet citées au début de cet article. La configuration retenue est résumée dans le tableau qui suit :

\begin{tabular}{lccc}
\hline \hline Paramètres libres & Notation & Qualifié & Non Qualifié \\
\hline \hline Coût de postage des emplois vacants & $c_{i}$ & 0,6 & 0,25 \\
Paramètre d'efficacité de la fonction d'appariement & $k_{i}$ & 3,175 & 2,75 \\
Taux d'arrivée des chocs de productivité & $\lambda_{i}$ & 0,125 & 0,2 \\
Borne inférieure de la productivité & $\varepsilon_{l_{i}}$ & $-0,375$ & $-0,425$ \\
\hline \hline
\end{tabular}

Dans ce cas, on obtient un taux de chômage global de $9,6 \%$, le taux de destruction moyen $\bar{q}$ vaut 9,3\% tandis que la durée moyenne du chômage est d'environ 11 mois. Ceci correspond à des taux de chômage par qualification valant respectivement 15, 25\% pour les non qualifiés et $6,4 \%$ pour les qualifiés, et des durées moyennes de chômage par qualification valant environ 9 mois et demi et 15 mois respectivement pour les qualifiés et les non qualifiés, tandis que les taux de destructions des emplois $q_{i}$ sont de $7,2 \%$ pour les 
qualifiés et $13 \%$ pour les non qualifiés. Enfin, la proportion de qualifiés est de $63,6 \%$, tandis que le taux de taxe $\tau$ obtenu est de $6,4 \%$, comme celui constaté. Il convient de noter que les valeurs des paramètres retenues assurent que les deux groupes de travailleurs restent employables, i.e. $\varepsilon_{c_{1}}<\varepsilon_{c_{2}}<\varepsilon_{u}$, tout en gardant à l'esprit qu'une des distorsions induite par l'introduction de rigidités renvoie à l'accroissement des problèmes d'employabilité auxquels sont confrontés les moins qualifiés. Ces résultats sont condensés dans le tableau qui suit :

\begin{tabular}{lcc}
\hline \hline Variables & Qualifié & Non Qualifié \\
\hline \hline Taux de chômage $u_{i}$ & $6,35 \%$ & $15,25 \%$ \\
Taux de destruction $q_{i}$ & $7,2 \%$ & $13 \%$ \\
Taux de sortie du chômage $\mu_{i}$ & 1,28 & 0,8 \\
Durée moyenne du chômage $\mu_{i}^{-1}$ & 9 mois et demi & 15 mois \\
Taux de taxe uniforme $\tau$ & $6,4 \%$ & $6,4 \%$ \\
Proportion de travailleurs de chaque type $P_{i}$ & $63,6 \%$ & $36,4 \%$ \\
\hline \hline
\end{tabular}

Dans la suite de cette section, nous partons de cette situation de référence pour décrire les effets respectifs des coûts de licenciement, de l'experience rating et enfin d'une substitution entre les deux dispositifs. Nous procédons pour cela en deux temps ; nous commençons dans chaque cas par décrire les effets de la LPE et de l'experience rating à qualification donnée dans les trois première sous-section, puis nous cherchons à comparer ces effets à qualification endogène vs. exogène dans la dernière sous-section.

\subsection{Les effets des coûts de licenciement}

Nous décrivons ici les effets associés à une variation du montant des coûts de licenciement à partir de la situation de référence, en raisonnant à qualification donnée. A des fins d'illustration, nous considérons ici l'impact d'un doublement de ceux-ci (i.e. l'indice $\gamma$ passe de 1 à 2). Les figures 1 a à $1 \mathrm{c}$ indiquent l'impact de ce changement sur les taux de chômage par qualification (figures 1a et 1b), et le taux de chômage global (figure 1c) , tandis que les figures $1 \mathrm{~d}$ à $1 \mathrm{i}$ illustrent les effets induits par ce changement sur les variables qui déterminent les taux d'entrée et de sortie du chômage, i.e. les seuils de licenciement $\varepsilon_{d_{i}}$ (figures $1 \mathrm{~d}$ et $1 \mathrm{e}$ ) et d'embauche $\varepsilon_{c_{i}}$ (figures $1 \mathrm{f}$ et $1 \mathrm{~g}$ ) ainsi que sur la tension sur chaque marché $\theta_{i}$ (figures $1 \mathrm{~h}$ et $1 \mathrm{i}$ ). Les figures $1 \mathrm{j}$ et $1 \mathrm{k}$ illustrent plus particulièrement les effets induits sur la taxation. Ainsi, la figure $1 \mathrm{j}$ s'intéresse aux effets sur le taux de taxe $\tau$, tandis que la figure $1 \mathrm{k}$ montre l'effet induit sur le montant du subventionnement $T_{2}=-T_{1}$ dont bénéficie le secteur non qualifié. Pour finir, la figure 11 s'intéresse aux effets induits sur la productivité moyenne des emplois de l'économie. 
Pour chacune de ces figures, nous représentons trois courbes : celle en trait continu correspond aux valeurs de notre benchmark $\left(s_{2}=0,835\right)$ tandis que les courbes en traits pointillés et en traits longs discontinus correspondent à des valeurs de la productivité des non qualifiés $s_{2}$ respectivement inférieures et supérieures de 7,5\% à la valeur de référence. Nous commençons par décrire les effets de la LPE correspondant aux courbes continues, avant de comparer les résultats pour des niveaux de productivité différents.

\section{[insérer ici les figures du panel 1]}

Les figures $1 \mathrm{a}$ et $1 \mathrm{~b}$ font ainsi ressortir des effets contrastés de la protection de l'emploi en fonction du niveau de qualification des individus. Nous remarquons que la hausse de la protection de l'emploi s'avère bénéfique pour le taux de chômage des qualifiés, qui diminue de 6,35 à 5,85\%. En revanche, l'impact sur les non qualifiés est clairement négatif : leur taux de chômage augmente d'environ un tiers de point de pourcentage, passant de 15,25 à 15,55\%. L'effet sur le chômage global est par contre incertain; avec la structure de la population active retenue, il s'avère légèrement favorable, puisque le taux de chômage global baisse d'environ 1/4 de point, passant de 9,6 à 9,40\%. Ces évolutions s'expliquent comme suit : comme nous pouvions nous y attendre, le renforcement de la LPE favorise la rétention de main-d'oeuvre sur chaque marché (baisse de $\varepsilon_{d_{i}}$ illustrée par les figures $1 \mathrm{~d}$ et $1 \mathrm{e}$ ), tandis que l'accroissement des rigidités s'accompagne d'une baisse notable des sorties du chômage, et ce, pour les qualifiés comme pour les non qualifiés. Cette chute des sorties est liée à deux phénomènes : la chute des créations d'emplois (baisse de $\theta_{i}$ dans chaque cas, comme illustré en $1 \mathrm{~h}$ et 1i), et l'élévation de la productivité de réserve à la création $\varepsilon_{c_{i}}$ (illustrée en $1 \mathrm{f}$ et $1 \mathrm{~g}$ ). Ainsi, au sein de chaque secteur, la hausse de la durée des épisodes de chômage est liée à des offres d'emplois moins nombreuses et refusées plus fréquemment du fait de la hausse des rigidités. Les évolutions différentes des taux de chômage par qualification s'expliquent alors par le fait que l'effet destruction -celui lié à la rétention de la main d'oeuvre- l'emporte pour les qualifiés, tandis que l'effet création -celui lié à la sortie du chômage- est plus important pour les non qualifiés. Il s'ensuit des évolutions opposées des taux de chômage par qualification et une hausse des inégalités de chômage.

La figure $1 \mathrm{j}$ révèle que l'effet de la LPE aurait pu être bien plus négatif sans la prise en compte de la taxation : l'effet de rétention de la main-d'oeuvre est renforcé, tandis que l'effet négatif sur les créations d'emplois est adouci par la baisse du taux de taxe $\tau$. En effet, ce taux baisse ici légèrement, de l'ordre de 0,03 point de pourcentage, suite au renforcement de la LPE, ce qui est favorable aux créations d'emplois et limite les destructions au sein de chaque secteur. 
Nous observons de surcroît que le subventionnement des qualifiés vers les non qualifiés augmente continûment. Il apparaît donc que l'impact de la LPE aurait été encore plus favorable pour les qualifiés en l'absence d'évolution endogène de la taxation et encore plus défavorable pour les non qualifiés, puisque les premiers supportent la charge supplémentaire liée à cette subvention, en l'absence de laquelle ils pourraient bénéficier d'une fiscalité plus favorable, alors que les seconds auraient été confrontés à une fiscalité plus contraignante s'ils n'avaient pas bénéficié de la hausse de cette subvention.

La LPE induit également une baisse de la productivité moyenne des emplois de cette économie, comme le montre la figure 1l, dans la mesure où celle-ci contribue à maintenir en place des emplois dont la productivité est faible et qui auraient été détruits en son absence.

Lorsque l'on s'intéresse aux courbes pointillées et discontinues afin de comparer les résultats correspondant à différents niveaux de productivité, il apparaît tout d'abord que ces résultats sont relativement robustes sur le plan qualitatif. Nous constatons également qu'une variation de la productivité des non qualifiés, $s_{2}$, affecte l'évolution du chômage des qualifiés puisque nous observons que les variations à la hausse (baisse) de $s_{2}$ entraînent une diminution (augmentation) du chômage des travailleurs qualifiés. Ceci tient au fait que les variations de $s_{2}$ affectent l'évolution de la taxation, et par ce biais, le marché des travailleurs qualifiés. Ceci illustre l'effet mis en évidence dans l'équation (38) de la section 5 .

Au total, si l'effet sur le chômage global semble faible, cela dissimule un impact très défavorable sur la productivité moyenne des emplois ainsi que sur la situation des travailleurs les moins qualifiés, dont le taux et la durée de chômage augmentent de façon notable, et dont l'employabilité pourrait également être menacée par l'accroissement des rigidités.

\subsection{Les effets de l'experience rating}

Il est également possible d'étudier, toujours à partir de la situation de référence, les effets associés à l'introduction d'une taxe sur les licenciements inspirée des systèmes d'experience rating. Précisément, nous nous intéressons désormais à l'effet d'une variation de l'indice de modulation, $e$, qui passe de 0 à 1 sans pour autant se substituer aux mesures traditionnelles de LPE. Le cas d'une substitution entre les deux dispositifs est envisagé dans la dernière sous-section.

L'accroissement de l'indice de modulation est susceptible d'induire deux phénomènes, le premier analogue à celui découlant d'une variation de la LPE traditionnelle et le second lié plus spécifiquement à la modulation des cotisations patronales à l'assurance chômage, i.e. (i) un accroissement des rigidités, puisque les taxes sur les licenciements augmentent avec l'indice de modulation $e$, ce qui limite les destructions mais décourage les créations 
d'emplois, et ce pour tous les marchés; (ii) une baisse du taux de taxe $\tau$ puisqu'à mesure que l'indice de modulation augmente la part du financement de l'assurance chômage financée par cette taxe diminue, ce qui permet à la fois de réduire les destructions et d'encourager les créations, et ce, sur l'ensemble des marchés. L'impact global est donc a priori incertain mais peut être évalué numériquement. Notons que l'effet mentionné en (i) a toutes les chances d'être important pour les non qualifiés, puisque la taxe sur les licenciements dépend en partie de la durée du chômage qui est plus élevée pour ces derniers.

Les figures qui suivent indiquent l'impact de cette réforme sur les taux de chômage par qualification (figures $2 \mathrm{a}$ et $2 \mathrm{~b}$ ), le taux de chômage global (figure 2c), sur les variables affectant les taux d'entrée et de sortie du chômage (figure $2 \mathrm{~d}$ à $2 \mathrm{i}$ ), sur la taxation (figures $2 \mathrm{j}$ et $2 \mathrm{k}$ ), et enfin sur la productivité moyenne des emplois (figure 2l). Comme pour la LPE, nous considérons trois courbes sur chaque graphique correspondant à différentes valeurs de la productivité, $s_{2}$, en considérant la courbe continue comme cas de référence.

\section{[insérer ici les figures du panel 2]}

A partir des figures $2 \mathrm{a}$ et $2 \mathrm{~b}$, Nous constatons que l'impact de la taxe sur les licenciements est clairement positif, et ce, pour le chômage des qualifiés comme pour celui des non qualifiés. Le taux de chômage des premiers baisse de 6,4 à 5,35\%, tandis que celui des seconds chute d'environ deux points, passant de 15,25 à 13,25\%. Avec la structure de la population active retenue, la baisse du taux de chômage global est d'environ un point de pourcentage et demi : celui-ci passe ainsi de 9,6 à 8,25\%. Ces évolutions s'expliquent comme suit. La hausse de l'indice de modulation limite les destructions d'emplois tout comme le ferait la LPE traditionnelle, et pour cette raison, les seuils de destructions $\varepsilon_{d_{1}}$ et $\varepsilon_{d_{2}}$ diminuent. Ce point est illustré sur les figures $2 \mathrm{~d}$ et $2 \mathrm{e}$. Les figures $2 \mathrm{f}$ à $2 \mathrm{i}$ montrent, quant à elles, l'effet induit sur les sorties du chômage qui s'avère contrasté. Pour les non qualifiés, la durée du chômage augmente, conformément aux craintes formulées par Fougère et Margolis (2000). Ceci est lié à l'accroissement du seuil d'embauche $\varepsilon_{c_{2}}$ qui s'accompagne ici d'une baisse de la tension $\theta_{2}$ : les non qualifiés voient donc leurs candidatures davantage rejetées suite à l'accroissement des rigidités, et font de plus face à une demande de travail qui diminue. Pour les qualifiés, l'effet sur les sorties est plus mitigé, puisque le seuil d'embauche $\varepsilon_{c_{1}}$ décrit une faible convexité par rapport à l'origine alors que la tension $\theta_{1}$ décrit une faible concavité par rapport à l'origine, le point de retournement de ces courbes correspondant à un indice de modulation d'environ 80\%. Leur taux de sortie commence donc par augmenter du fait d'une demande de travail plus forte et de rencontres débouchant plus fréquemment sur des embauches, avant que le phénomène ne s'inverse. Cette ambiguïté découle du fait que les qualifiés bénéficient d'un côté de la 
baisse du taux de taxe $\tau$ comme le montre la figure $2 \mathrm{j}$, mais d'un autre côté, l'effet lié à l'accroissement des rigidités qui accompagne la hausse de $e$ finit par peser de manière importante lorsque l'indice de modulation augmente. Cet effet finit par dominer pour un indice de modulation supérieur à environ 0,8 et les sorties du chômage s'en ressentent. Pour les non qualifiés, l'effet fiscal favorable lié à la baisse du taux de taxe $\tau$ est toujours dominé par l'effet défavorable lié à l'accroissement des rigidités.

Il est également possible de constater la baisse du subventionnement dont bénéficiait ce secteur aux dépens du secteur qualifié, comme le montre la figure $2 \mathrm{k}$. L'augmentation de l'indice de modulation s'accompagne donc ici d'un accroissement de la contribution des travailleurs peu qualifiés au financement de l'assurance chômage. Il convient en outre de remarquer que la subvention implicite entre les segments du marché du travail ne disparaît pas complètement lorsque l'indice de modulation, $e$, tend vers l'unité. De fait, nous retrouvons, ici, une des conclusions de Feldstein (1976) selon laquelle il faut un indice de modulation supérieur à l'unité afin de corriger les distorsions présentes dans l'économie, et plus particulièrement dans notre modèle afin de compenser la myopie dont fait preuve la caisse d'allocation chômage en ne tenant pas compte des trajectoires ultérieures des chercheurs d'emplois qui sortent de l'indemnisation du chômage. L'effet induit par la taxe d'experience rating sur la productivité moyenne des emplois est clairement défavorable à structure par qualification de la population donnée comme le montre la figures 21 .

Comme précédemment, nous comparons les résultats pour trois courbes : celle en trait continu correspond au benchmark, celle en traits pointillés à une valeur plus faible de $s_{2}$, et celle en traits longs discontinus à une valeur élevée de $s_{2}$. La comparaison révèle que les effets décrits sont relativement robustes sur le plan qualitatif à ces alternatives. Ainsi, nous constations qu'une variation de $s_{2}$ affecte l'équilibre du marché qualifié au travers le taux de taxe, $\tau$. Toutefois, l'impact d'une variation de $s_{2}$ sur l'évolution du marché qualifié apparaît plus faible en présence qu'en l'absence d'experience rating, dans la mesure où cette taxe a précisément pour effet de réduire le subventionnement et donc les interactions entre les deux segments du marché du travail. Ceci est particulièrement frappant lorsque l'on considère l'évolution de la tension $\theta_{1}$. En effet, il apparaît que la tension $\theta_{1}$ est d'autant plus forte que la productivité $s_{2}$ est élevée mais l'écart entre les trois courbes se resserre lorsque l'on s'approche d'un degré d'experience rating parfait.

Au final, il apparaît que l'introduction d'une taxe sur les licenciements inspirée du système d'experience rating a des effets mitigés : elle peut entraîner une baisse du chômage pour les qualifiés comme pour les non qualifiés; en contrepartie, elle est également susceptible de s'accompagner d'un accroissement notable de la durée du chômage, et la productivité moyenne des emplois diminue à qualification donnée, surtout si ce système s'ajoute aux rigidités existantes.

Dans la section qui suit, nous montrons qu'opérer une substitution parfaite entre 
experience rating et mesure traditionnelle de la LPE permettrait, tout en maintenant le niveau de protection de l'emploi inchangé, d'améliorer de façon significative la performance du marché du travail, pour toutes les catégories de travailleurs.

\subsection{Substituer l'expérience rating au système de protection de l'emploi actuel}

Nous nous intéressons ici aux effets liés à une substitution parfaite entre experience rating et protection de l'emploi. Précisément, à mesure que l'indice de modulation $e$ augmente, les coûts administratifs de licenciement sont réduits d'un montant tel que $d f_{i}=-d \tau_{e_{i}}$. De la sorte, le degré de rigidité n'augmente pas à mesure que l'indice de modulation progresse ${ }^{20}$. Les seuls effets liés à l'expérience rating sont les effets mobilisés par les variations de la fiscalité.

Les figures qui suivent indiquent l'impact de cette réforme sur les taux de chômage par qualification (figures 3a et 3b), sur le taux de chômage global (figure 3c), sur les variables affectant les taux d'entrée et de sortie du chômage (figures $3 \mathrm{~d}$ à $3 \mathrm{i}$ ), sur la taxation (figures $3 \mathrm{j}$ et $3 \mathrm{k}$ ) ainsi sur la productivité moyenne des emplois (figure 3l). Comme dans les cas précédents, nous représentons sur chaque graphiques trois courbes correspondant à des valeurs hautes (traits longs discontinus), moyennes (trait continu), et basses (traits pointillés) de la productivité $s_{2}$.

[insérer ici les figures du panel 3]

Il ressort que l'effet d'une telle substitution s'avère favorable, que ce soit sur le chômage des qualifiés qui baisse d'environ $1 / 3$ de point, passant de 6,35 à $6,0 \%$ (figure 3a), que sur celui des non qualifiés qui baisse de plus d'un point, passant de 15,25 à 13,75\% (figure 3b) ou sur le chômage global qui diminue ici d'environ 8/10 de point, passant de 9,6 à 8,8 \% (figure 3c). Au regard de la section précédente, le point intéressant est ici que la baisse du chômage est liée à la fois à une diminution des destructions d'emplois comme le montrent les figures $3 \mathrm{~d}$ et $3 \mathrm{e}$, et à une progression des créations pour chaque type de qualification comme le montrent les figures $3 \mathrm{f}$ à 3 i. En effet, la substitution stimule les créations d'emplois comme l'indiquent les figures $3 \mathrm{~h}$ et $3 \mathrm{i}$ où l'on voit que $\theta_{1}$ et $\theta_{2}$ augmentent, et de plus une plus grande proportion des rencontres devient acceptable, puisque comme le montrent les figures $3 \mathrm{f}$ et $3 \mathrm{~g}$, les seuils $\varepsilon_{c_{1}}$ et $\varepsilon_{c_{2}}$ diminuent. Au total,

20. Plus généralement, il est possible de considérer $d f_{i}=-g * d \tau_{e_{i}}$, où le paramètre $g$ traduirait le degré de substitution entre les deux dispositifs de protection de l'emploi. La sous-section précédente correspond au cas où $g=0$ tandis qu'ici $g=1$. La valeur de ce paramètre conditionne ainsi l'importance de la hausse des rigidités associée à la taxe sur les licenciements. 
la durée du chômage est donc plus courte, et ce, pour chaque groupe de qualification. L'effet fiscal lié à la baisse du taux de taxe $\tau$ (figure 3j) joue ici à plein pour stimuler les sorties du chômage, et ce, sans être contrecarré par un accroissement des rigidités. La figure 3k montre que le subventionnement qui s'opérait jusque là en faveur des non qualifiés se réduit à mesure que l'indice de modulation $e$ augmente. Enfin, comme dans les cas précédents la productivité moyenne tend à diminuer mais cette baisse est moins marquée.

Les effets décrits ici demeurent sur le plan qualitatif quand nous faisons varier la productivité $s_{2}$. Les variations de $s_{2}$ affectent l'équilibre du marché des travailleurs qualifiés mais l'on constate toutefois que cet effet s'estompe à mesure que le degré d'experience rating augmente.

Il est donc possible d'améliorer l'efficacité du fonctionnement du marché du travail sans réduire le degré de protection de l'emploi global. Ces divers éléments montrent bien les différences qualitatives qui peuvent exister entre les deux modes de protection de l'emploi et suggère également que l'impact de l'experience rating sur la sortie du chômage dépend de la capacité à substituer totalement ou non ce système à la protection de l'emploi telle qu'elle prévaut actuellement sur le marché du travail.

\subsection{Effets à éducation endogène vs. exogène}

Nous avons présenté jusque-là les effets de la LPE à qualification donnée. Nous cherchons ici à comparer ses effets selon que la structure par qualification de la population soit endogène ou exogène dans les trois cas qui nous intéressent : hausse des coûts de licenciement traditionnel (panel 4), instauration de l'experience rating (panel 5), substitution entre LPE traditionnelle et experience rating (panel 6). A cette fin, nous représentons les effets de la LPE sur les taux de chômage par qualification $u_{1}$ et $u_{2}$ et le taux de chômage agrégé (figures $a, b$ et $c$ de chaque panel), la proportion de qualifiés $P_{1}$ (figure $d$ ). Afin de savoir si l'évolution de cette proportion est liée à une hausse ou à une baisse du bien-être attendu par chaque catégorie, nous retraçons l'évolution de $r V_{u_{1}}$ et $r V_{u_{2}}$ dans les figures $e$ et $f$. Enfin, les figures $g$ à $i$ décrivent l'évolution de la productivité moyenne de chaque type d'emploi et la productivité moyenne globale.

Sur chaque graphique, la courbe en trait continu représente l'évolution de la variable d'intérêt à qualification donnée, tandis que la courbe en traits pointillés décrit l'évolution de cette même variable à qualification endogène.

LPE traditionnelle. Les effets des coûts de licenciement étaient relativement défavorables à qualification donnée, en particulier pour les moins qualifiés. Lorsque la proportion de travailleurs qualifiés évoluent de manière endogène, les effets négatifs de la LPE sont en partie amoindris du fait de l'augmentation de la proportion de travailleurs qualifiés, 
même si les différences dans les évolutions de certaines variables entre éducation endogène et exogène sont parfois limitées. Les contrastes les plus notables concernent l'évolution du chômage agrégé et de la productivité moyenne comme le montre le panel de figures qui suit.

\section{[insérer ici les figures du panel 4]}

D'après les figures $4 \mathrm{a}$ à $4 \mathrm{c}$, nous constatons que la hausse du niveau de qualification permet une baisse du chômage plus importante qu'à éducation donnée. En effet, comme les travailleurs qualifiés sont par essence moins exposés au risque de chômage, le taux de chômage agrégé diminue mécaniquement à mesure que la proportion de qualifiés s'accroît. En outre, la diminution du chômage induit alors une baisse du taux de taxe $\tau$ qui se révèle également favorable à l'emploi des non qualifiés comme l'atteste la figure 4b.

D'après les figures $4 \mathrm{e}$ et $4 \mathrm{f}$, la hausse de la proportion de qualifiés $P_{1}$ décrite en $4 \mathrm{~d}$ découle elle-même d'une détérioration de l'utilité de réserve des qualifiés comme de celle des non qualifiés, mais les perspectives des premiers se dégradent moins que celles des seconds. Cette hausse du nombre de travailleurs qualifiés n'est ici cependant pas suffisante pour permettre une augmentation de la productivité moyenne des emplois, qui évolue de manière défavorable à éducation endogène comme exogène, même si cette évolution est légèrement moins défavorable dans le premier cas.

Experience Rating. Les effets de l'experience rating sont plus prononcés lorsque les qualifications sont endogènes plutôt qu'exogènes, comme le montre le panel ci-dessous.

\section{[insérer ici les figures du panel 5]}

La taxe d'experience rating a un fort impact sur les incitations à s'éduquer, puisque la proportion de travailleurs éduqués augmente de près de 10 points, comme le montre en particulier la figure $5 \mathrm{~d}$.

Cette hausse du nombre de travailleurs qualifiés correspond à une amélioration notable de la situation des qualifiés, mais à une dégradation de l'utilité de réserve des chômeurs peu qualifiés. Lorsque la structure par qualification de la population est endogène, l'impact de l'experience rating sur l'évolution du chômage global est ainsi encore très marquée, puisque le taux de chômage global baisse encore davantage qu'à éducation exogène : la hausse de 10 points du pourcentage de travailleurs qualifiés dans la population permet ici une baisse de 1 point supplémentaire du taux de chômage agrégé par rapport à la situation de référence. Le taux de chômage global passe ainsi de 9,6 à 7,25\% contre 8,25\% à qualification donnée, comme le révèle la figure 5c. 
L'impact de l'experience rating sur la productivité moyenne des emplois diffère ici selon que la qualification est endogène ou exogène : l'impact est clairement défavorable à structure par qualification de la population donnée, comme le montre la figures $5 \mathrm{i}$; en revanche il est favorable lorsque les décisions d'éducation sont endogènes. L'experience rating incite à s'éduquer un nombre suffisamment important de travailleurs pour contrebalancer l'évolution défavorable de la productivité sur chaque type d'emploi comme l'attestent les figures $5 \mathrm{~g}, 5 \mathrm{~h}$ et $5 \mathrm{i}$.

Substitution entre experience rating et LPE. A éducation endogène, il apparaît que l'impact bénéfique de l'experience rating demeure lorsque l'on substitue ce mode de protection de l'emploi à la LPE traditionnelle, comme le montre le panel qui suit.

\section{[insérer ici les figures du panel 6]}

La substitution entre les deux modes de protection de l'emploi induit également un accroissement du nombre de travailleurs qualifiés. Cette augmentation du niveau de qualification de la population est ici plus modeste par rapport au cas précédent. Cependant, ceci s'explique par le fait que l'utilité de réserve des chômeurs qualifiés, tout comme celle des non qualifiés augmentent simultanément.

Lorsque l'on compare les effets de la substitution à éducation exogène vs. endogène, il apparaît que la hausse du niveau de qualification tend à amplifier l'impact favorable de la substitution sur le chômage, en particulier si l'on considère la figure 6c. Le taux de chômage global passe ainsi de 9,6 à $8,5 \%$ contre $8,8 \%$ à qualification donnée. Le caractère endogène ou non du niveau de qualification affecte en revanche de manière importante l'évolution de la productivité de cette économie, puisqu'à structure par qualification de la population donnée, la productivité moyenne des emplois diminue, comme le montre la figure $6 \mathrm{i}$, alors qu'à qualification endogène, la productivité moyenne s'améliore. En d'autres termes, la hausse du niveau de qualification est ici suffisamment importante pour contrebalancer la hausse de la productivité moyenne de chaque type d'emploi, décrite par les figures $6 \mathrm{~g}$ et $6 \mathrm{~h}$.

\section{Discussion}

Dans cette section, nous discutons deux limites potentielles de notre travail. La première limite a trait au mode de formation des salaires pour les travailleurs non qualifiés, la seconde aux contraintes financières auxquelles sont confrontées les firmes lorsque la protection de l'emploi est importante. 
Salaire minimum. Nous avons supposé au fil de cet article que les salaires étaient librement négociés, une hypothèse contestable en ce qui concerne les travailleurs peu qualifiés. Notre étude suggère que les effets de la LPE sont susceptibles d'être nefastes aux moins qualifiés, même dans le cadre a priori le plus favorable où les salaires sont négociés, et s'ajustent de ce fait aux conditions du marché du travail. L'introduction d'un salaire minimum contraignant ne ferait vraisemblablement que rendre la LPE encore plus défavorable pour ces travailleurs ${ }^{21}$. En ce qui concerne les effets de l'experience rating, nous renvoyons ici le lecteur à l'article de Cahuc et Malherbet (2004) qui montre que même en présence de coûts de licenciement élevés et d'un salaire minimum contraignant, l'introduction de la taxe sur les licenciement reste désirable pour les travailleurs non qualifiés. Nous avons cherché à compléter cette étude en montrant que certaines des conséquences défavorables de l'experience rating, notamment sur la durée du chômage des moins qualifiés, pouvaient être évitées dès lors que la taxe sur les licenciement se substitue de manière suffisamment large à la LPE existante. Cette conclusion serait-elle remise en cause par la présence d'un salaire minimum contraignant? Dans la mesure où une substitution parfaite entre experience rating et LPE n'implique aucune augmentation des rigidités, mais seulement un effet fiscal favorable, nous pensons que notre résultat devrait être robuste à l'introduction d'un salaire minimum. En revanche, il semble évident que si la substitution entre les deux modalités de protection de l'emploi n'est pas parfaite ou insuffisante, il est possible que l'effet fiscal favorable lié au système d'experience rating soit largement dominé par la hausse des rigidités.

D'autres effets liés à l'introduction d'un salaire minimum contraignant dans notre modèle méritent d'être mentionnés. La principale d'entre elles est que dans un cadre à plusieurs secteurs reliés par le biais d'une contrainte budgétaire comme le notre, il apparaît que l'existence d'un salaire minimum pour les travailleurs non qualifiés pourrait avoir des répercussions pour les travailleurs qualifiés à travers le système fiscal. Pour s'en convaincre, réécrivons la contrainte de financement de l'assurance chômage. En notant, $\underline{w}$, le salaire minimum, cette contrainte s'écrit désormais :

$$
\tau=\frac{\sum_{i} b_{i} U_{i}-\sum_{i} q_{i} E_{i} \tau_{e_{i}}}{n_{o_{1}} \bar{w}_{o_{1}}+n_{e_{1}} \bar{w}_{e_{1}}+\left(n_{o_{2}}+n_{e_{2}}\right) \underline{w}}
$$

Si l'on vérifie que $\frac{d \tau}{d \underline{w}} \geq 0$, ce qui est possible dans la mesure où $\underline{w}$ affecte ici positivement le chômage et négativement l'emploi à participation donnée, il serait alors possible de

21. Notons cependant que la prise en compte d'un salaire minimum dans le cadre d'un modèle d'appariement à destruction d'emplois endogène demeure une tâche complexe ayant fait l'objet d'études spécifiques par Cahuc et Zylberberg (1999, 2004) ou encore Garibaldi et Violante (2004) et dépasse le cadre de ce papier. 
montrer que dans ce cadre :

$$
\frac{d \theta_{1}}{d \underline{w}}=\frac{d \theta_{1}}{d \tau} \frac{d \tau}{d \underline{w}} \leq 0 ; \frac{d \varepsilon_{c_{1}}}{d \underline{w}}=\frac{d \varepsilon_{c_{1}}}{d \tau} \frac{d \tau}{d(+)} \frac{d \underline{w}}{(+)} \geq 0 ; \frac{d \varepsilon_{d_{1}}}{d \underline{w}}=\frac{d \varepsilon_{d_{i}}}{d \tau} \frac{d \tau}{d \underline{w}} \geq 0
$$

De sorte que :

$$
\frac{d u_{1}}{d \underline{w}}=\frac{d u_{1}}{d \theta_{1}} \frac{d \theta_{1}}{d \underline{w}}+\frac{d u_{1}}{d \varepsilon_{c_{1}}} \frac{d \varepsilon_{c_{1}}}{d \underline{w}}+\frac{d u_{1}}{d \varepsilon_{d_{1}}} \frac{d \varepsilon_{d_{1}}}{d \underline{w}} \geq 0
$$

Ainsi, une hausse du salaire des non qualifiés $\underline{w}$ pourrait ici avoir des conséquences non seulement sur l'équilibre du marché non qualifié, mais aussi se répercuter sur le marché qualifié via la contrainte budgétaire. Suite à une hausse de $\underline{w}$, le chômage sur le marché non qualifié est ainsi susceptible d'augmenter, entraînant une hausse du taux de taxe $\tau$ qui induit à son tour des conséquences négatives quant à l'évolution de l'emploi sur le marché qualifié. Une multiplicité d'équilibres est susceptible d'apparaître du fait de ce mécanisme. Le point nouveau est ici que du fait des interdépendances entre les deux marchés découlant de la contrainte budgétaire, une faible variation du salaire $\underline{w}$ peut entraîner des changements importants en faisant basculer l'économie d'un équilibre à fort taux d'emploi, faible taxation sur chaque marché à un équilibre à faible niveau d'emploi et forte taxation.

De surcroît, en détériorant la situation des non qualifiés, ce salaire minimum est susceptible d'encourager un plus grand nombre de travailleurs à s'éduquer ${ }^{22}$. Ceci limiterait les conséquences néfastes pour l'emploi liées à ce salaire minimum et permettrait de stimuler la productivité des emplois de cette économie.

Contraintes financières. Nous avons supposé, à l'instar de la majorité de la littérature, que les firmes étaient neutres au risque et n'étaient pas contraintes financièrement. Ces deux hypothèses renvoient à deux hypothèses implicites des modèles d'appariement : (i) les marchés financiers sont supposés parfaits. Ainsi, les firmes peuvent complètement diversifier leurs actifs et s'assurer parfaitement; (ii) les firmes peuvent toujours faire face à leurs engagements. Elles sont ainsi supposées avoir "les poches profondes" (deep pockets). Bien que commune dans la littérature, ces hypothèses sont de toute évidence restrictives ${ }^{23}$. En particulier, l'introduction d'un système d'experience rating n'est-il pas de nature à introduire des distorsions supplémentaires lorsque les firmes sont contraintes sur le marché du crédit? Pour lever ces hypothèses, il est nécessaire de considérablement amender le cadre du modèle en introduisant par exemple des imperfections sur le marché du crédits à l'instar de Wasmer et Weil (2004), ce qui dépasse largement le cadre de cet article et constituent un axe de recherche à part entière. Pour autant, il est possible de capturer

22. Un argument similaire concernant l'impact du salaire minimum sur les incitations à s'éduquer est établi dans un cadre différent par Cahuc et Michel (1996).

23. Voir par exemple Blanchard et Tirole (2008) pour une discussion. 
les implications essentielles liées aux contraintes financières en supposant que les firmes disposent de fonds limités pour satisfaire aux exigences de la LPE. D'un point de vue formel cela revient à remplacer dans les équations d'équilibre les termes $f_{i}+\tau_{e_{i}}$ par $\min \left(\bar{f}, f_{i}+\tau_{e_{i}}\right)$ où $\bar{f}$ est la contrainte financière de la firme, i.e. l'engagement maximum auquel elle peut satisfaire ${ }^{24}$. Que peut-on attendre d'une telle contrainte financière? Le résultat est assez intuitif. La contrainte financière induit une distortion supplémentaire dans les décisions de destruction des emplois des firmes lorsque la contrainte est saturée. Les entreprises tendent alors à détruire trop d'emplois puisqu'elles ne sont plus en mesure d'internaliser complètement le coût de leurs licenciements. Il s'ensuit que pour un indice de modulation, e, donné, le taux de destruction des emplois est plus élevé que dans le cadre de référence, et ce d'autant plus que la contrainte financière est forte ( $\bar{f}$ faible). Une seconde distortion apparaît par le système fiscal. Les firmes ne satisfaisant plus à leurs engagements, il est nécessaire d'augmenter la taxe sur la masse salariale pour équilibrer les ressources et les dépenses de la caisse d'assurance chômage. La hausse de la fiscalité réduit la profitabilité de tous les emplois et limite les créations d'emplois. Au final, le taux de chômage est plus important que dans la situation de référence. Les résultats que nous obtiendrions dans le cadre de notre modèle seraient alors analogues à ceux de Blanchard et Tirole (2008) qui soulignent l'importance des contraintes financières auxquelles sont confrontées les firmes lorsque la LPE s'avère contraignante. Pour autant, dans la mesure où nous considérons, in fine, une substitution parfaite entre la LPE traditionnelle et l'experience rating de telle sorte que $d \tau_{e_{i}}=-d f_{i}$, l'introduction d'une taxe d'experience rating n'est pas à même de contraindre plus fortement les firmes que les modalités de protection de l'emploi pré-existantes.

\section{Conclusion}

Dans cet article, nous avons cherché à mettre en évidence les effets liés à l'introduction d'un système de modulation des cotisations patronales à l'assurance chômage adaptée du système américain d'experience rating lorsque la main-d'oeuvre est hétérogène. Nous montrons que l'introduction d'un système d'experience rating peut être favorable en terme de chômage agrégé et par qualification, mais que ce constat mérite d'être nuancé lorsque l'on s'intéresse à la durée du chômage, en particulier pour les non qualifiés. A court terme, l'importance de cet effet indésirable dépend de la capacité à substituer la modulation des cotisations patronales à l'assurance chômage à la protection de l'emploi existante ou non. Une substitution complète permettrait ainsi d'améliorer les performances du marché du travail sans qu'il soit nécessaire de réduire le degré de protection de l'emploi. A plus

24. Blanchard et Tirole (2008) adoptent une hypothèse similaire. Il est à noter que dans notre cadre lorsque $\bar{f}=f_{i}+\tau_{e_{i}}$ nous retrouvons le modèle de base. 
long terme, la proportion de travailleurs qualifiés évolue en fonction des conditions qui prévalent sur le marché du travail, ce qui permet de réduire les éventuels effets néfastes associés à une réforme de la LPE.

\section{Références}

[1] Albrecht, J., et Vroman, S., 1999, "Unemployment Compensation Finance and Efficiency Wages", Journal of Labor Economics 17, 141-167.

[2] Anderson, P., et Meyer, B., 2000, "The Effects Of the Unemployment Insurance Payroll Tax On Wages, Employment, Claims And Denials", Journal of Public Economics 78, 81-106.

[3] Bertola, G., 2004, "A Pure Theory of Job Security and Labour Income Risk", Review of Economic Studies, 71, 43-61.

[4] Blanchard O. J. et Diamond, P., 1989. "The Beveridge Curve", Brookings Papers on Economic Activity, 1, 1-60

[5] Blanchard, O.J., 2000, "Employment protection, sclerosis, and the effects of shocks on unemployment", Lionel Robins Lecture, (Lecture 3).

[6] Blanchard O.J., et Tirole, J., 2003, "Protection de l'emploi et procédures de licenciement", Rapport pour le Conseil d'Analyse Economique 44, Paris : La Documentation française.

[7] Blanchard O.J., et Tirole, J., 2008, "The Optimal Design of Unemployment Insurance and Employment Protection. A First Pass", Journal of the European Economic Association, à paraître.

[8] Boeri, T., et Garibaldi, P., 2006, "Two Tier Reforms of Employment Protection Legislation : a Honeymoon Effect?" Université Bocconi, miméo.

[9] Burdett, K., et Wright, R., 1989, "Optimal Firm Size, Taxes, and Unemployment", Journal of Public Economics 39, 275-287.

[10] Cahuc, P., 2003, "Pour une meilleure protection de l'emploi", Document de Travail du COE.

[11] Cahuc, P., et Malherbet, F., 2004, "Unemployment Compensation Finance and Labour Market Rigidity", Journal of Public Economics 88, 481-501.

[12] Cahuc, P., et Michel, P., 1996, "Minimum wage unemployment and growth ", European Economic Review 40, 1463-1482

[13] Cahuc, P., et Zylberberg, A., 1999, "Job Protection, Minimum Wage and Unemployment", IZA working paper 95. 
[14] Cahuc, P., et Zylberberg, A., 2008, "Optimum Income Taxation and Layoff Taxes", IZA working paper 1678, forthcoming Journal of Public Economics

[15] Camdessus, M., 2004, "Le sursaut - Vers une nouvelle croissance pour la France", La documentation française, Paris.

[16] Card, D., et , Levine, 1994, "Unemployment Insurance Taxes and the Cyclical and Seasonal Properties of Unemployment", Journal of Public Economics 53, 1-29.

[17] Charlot, O., 2006, "Education, destruction des emplois et performance du marché du travail dans un modèle d'appariement", Revue Economique 57, 5-33.

[18] Cohen, D., Lefranc, A., et Saint-Paul, G., 1997, "French Unemployment : a Transatlantic Perspective", Economic Policy 25, 267-291.

[19] Commission Européenne, 2003, 2005, Décision du conseil relative aux lignes directrices pour les politiques de l'emploi des Etats membres, http ://ec.europa.eu/employment_social/employment_strategy/prop_2003/gl_fr.pdf.

[20] Crépon, B., Deniau N., et Perrez-Duarte, S., 2002, "Wages, Productivity, and Workers Characteristics : A French Perspective", mimeo CREST.

[21] Deere, D., (1991), "Unemployment Insurance and Employment", Journal of Labor Economics 9, 307-324.

[22] Devine, T.J., et Kiefer, D.H., 1991, Empirical Economics : the search approach, Oxford University Press.

[23] Drèze, J H., et Sneessens, H., 1997, "Technological Development, Competition from Low-Wage Economies and Low-Skilled Unemployment", in D.J. Snower et G. de la Dehesa, eds., Unemployment Policy : Government Options for the Labour Market, Cambridge : Cambridge University Press.

[24] Duhautois, R., 2002, "Les réallocations d'emplois sont-elles en phase avec le cycle?" Economie et Statistique 351, 87-104

[25] Esping-Andersen, G., 2000, "Who is Harmed by Employment Regulation?", dans Esping-Andersen, G. et M. Regini (eds), Why de-regulate labour markets, Oxford : Oxford University Press.

[26] Fath, J., et Fuest, C., 2005, "Experience Rating versus Employment Protection Laws in a Model where Firms Monitor Workers", Scandinavian Journal of Economics 107, 299-314.

[27] Feldstein, M., 1976, "Temporary Layoffs in the Theory of Unemployment", Journal of Political Economy 84, 937-957.

[28] Fougère, D., et Margolis, D., 2000, "Moduler les cotisations patronales à l'assurance chômage : les expériences de bonus-malus aux Etats-Unis", Revue Française d'Economie. 
[29] Garibaldi, P., et Violante, G., 2004, "The employment effects of severance payments with wage rigidities", Economic Journal 115, 799-832.

[30] Givord, P., et Maurin, E., 2004, "Changes in Job Stability and their Causes : an Empirical Analysis Method Applied to France 1982-2000", European Economic Review 48, 595-615.

[31] Holmlund, B., 1998, "Unemployment Insurance in Theory and Practice", Scandinavian Journal of Economics, 100, 113-141.

[32] Kramarz, F., et Michaud, M.-L., 2004, "The Shape of Hiring and Separation Costs", Working paper IZA 1170.

[33] Lazear, E., 1990, "Job Security Provision and Employment", Quarterly Journal of Economics, 105, 699-726.

[34] Ljungqvist, L., 2002, "How Do Layoff Costs Affect Employment?", Economic Journal, 112, 829-853.

[35] Malherbet, F., (2003), Modulation des cotisations patronales à l'assurance chômage, protection de l'emploi et performance du marché du travail, Thèse de doctorat, Université Paris 1 - Panthéon - Sorbonne.

[36] Malinvaud, E., 1998, "Les cotisations sociales à la charge des employeurs : analyse économique", Rapport pour le Conseil d'Analyse Economique, Paris : La Documentation française.

[37] Manacorda, M., et Petrongolo, B., 1999, "Skill Mismatch and Unemployment in OECD Countries", Economica 66, 181-207.

[38] Marceau, N., 1993, "Unemployment Insurance and Market Structure", Journal of Public Economics 52, 237-249.

[39] Millard, D., et Mortensen, D., 1997, "The Unemployment and Welfare Effects of Labour Market Policy : a Comparison of the US and the UK", in D.J. Snower et G. de la Dehesa, eds., Unemployment Policy : Government Options for the Labour Market, Cambridge : Cambridge University Press.

[40] Mincer, J., 1991, "Education and Unemployment", NBER working paper.

[41] Mongrain, S., et Roberts, J., 2005, "Unemploymentt Insurance and Experience Rating : Insurance vs Efficiency”, International Economic Review 46 , 1303-1319.

[42] Mortensen, D., et Pissarides, C., 1994, "Job Creation and Job Destruction in the Theory of Unemployment", Review of Economic Studies 61, 397-415.

[43] Mortensen, D., et Pissarides, C., 1999, "Unemployment Responses to Skill-biased Technology Shocks : the Role of Labour Market Policy", Economic Journal 109, 242-65. 
[44] Mortensen, D., et Pissarides, C., 1999b, "New developments in models of search in the labor market. Handbook of Labor Economics vol 3B, chapter 39, Ashenfelter, O. and Card, D. (eds), Elsevier Science Publisher.

[45] Mortensen, D., et Pissarides, C., 2003, "Tax, Subsidies and Labour Market Outcomes", dans Phelps E. (ed.), Designing Inclusion : tools to raise low-end pay and employment in private entreprise, Cambridge University Press.

[46] Nickell, S., 1979, "Education and the Life Cycle Pattern of Unemployment", Journal of Political Economy 87, 117-131.

[47] Nickell,S., Bell, B., 1997, "Would Cutting Payroll Taxes on the Unskilled have a Significant Impact on Unemployment?", in Snower and de la Dehesa (eds.), Unemployment policy : how should governments respond to unemployment?, Oxford University Press.

[48] OCDE, Perspectives de l'emploi, OCDE, Paris

[49] Petrongolo, B., et Pissarides, C., 2001, "Looking into the Black Box : A Survey of the Matching Function", Journal of Economic Litterature, 39, 390-441.

[50] Phelps, E.S., 1994, "Low-Wage Employment Subsidies versus the Welfare State", American Economic Review 84, 54-58

[51] Pissarides, C., 2000, Equilibrium Unemployment Theory, 2nde édition, Cambridge : MIT Press.

[52] Pissarides, C., 2001, "Employment protection", Labour Economics 8, 131-159.

[53] Saint-Paul, G., 1996, Dual labor market : a macroeconomic perspective : MIT Press

[54] Saint-Paul, G., 2002, The Political Economy of Labour Market Institutions : Oxford University Press.

[55] Topel, R., et Welsh, F., 1980, "Unemployment insurance : survey and extension", Economica 47, 351-379.

[56] Wasmer, E., 2006. General vs specific skills in labor markets with search frictions and firing costs. American Economic Review 96, 811-831.

[57] Wasmer, E., Weil, P., 2004. The macroeconomics of labor and credit market imperfections. American Economic Review 94, 944-963

\section{Annexes}

\section{Annexe 5.1 Calculs intermédiaires : salaires négociés}

Négociation salariale. Les salaires sont solutions des programmes suivants :

$$
\underset{w_{o_{i}}}{\operatorname{Max}}\left(1-\beta_{i}\right) \ln \left[\Pi_{o_{i}}(\varepsilon)-\Pi_{v_{i}}\right]+\beta_{i} \ln \left[V_{o_{i}}(\varepsilon)-V_{u_{i}}\right]
$$




$$
\underset{w_{e_{i}}}{\operatorname{Max}}\left(1-\beta_{i}\right) \ln \left[\Pi_{e_{i}}(\varepsilon)-\Pi_{v_{i}}+f_{i}+\tau_{e_{i}}\right]+\beta_{i} \ln \left[V_{e_{i}}(\varepsilon)-V_{u_{i}}\right]
$$

Les conditions du premier ordre impliquent :

$$
\begin{gathered}
\left(1-\beta_{i}\right) \frac{\Pi_{o_{i}}^{\prime}}{\Pi_{o_{i}}(\varepsilon)-\Pi_{v_{i}}}+\beta_{i} \frac{V_{o_{i}}^{\prime}}{V_{o_{i}}(\varepsilon)-V_{u_{i}}}=0 \\
\left(1-\beta_{i}\right) \frac{\Pi_{e_{i}}^{\prime}}{\Pi_{e_{i}}(\varepsilon)-\Pi_{v_{i}}+f_{i}+\tau_{e_{i}}}+\beta_{i} \frac{V_{e_{i}}^{\prime}}{V_{e_{i}}(\varepsilon)-V_{u_{i}}}=0
\end{gathered}
$$

Tenant compte des expressions des valeurs (5) à (9), nous obtenons $\Pi_{o_{i}}^{\prime}=-(1+\tau)$; $V_{o_{i}}^{\prime}=1 ; \Pi_{e_{i}}^{\prime}=-(1+\tau)$ et $V_{e_{i}}^{\prime}=1$. D'où les règles de partage suivantes :

$$
\begin{gathered}
\left(1-\beta_{i}\right)(1+\tau)\left[V_{o_{i}}(\varepsilon)-V_{u_{i}}\right]=\beta_{i}\left[\Pi_{o_{i}}(\varepsilon)-\Pi_{v_{i}}\right] \\
\left(1-\beta_{i}\right)(1+\tau)\left[V_{e_{i}}(\varepsilon)-V_{u_{i}}\right]=\beta_{i}\left[\Pi_{e_{i}}(\varepsilon)-\Pi_{v_{i}}+f_{i}+\tau_{e_{i}}\right]
\end{gathered}
$$

Il est alors possible de réécrire ces expressions en tenant compte des définitions des surplus (1) et (2), ce qui donne :

$$
\begin{gathered}
V_{o_{i}}(\varepsilon)-V_{u_{i}}=\frac{\beta_{i}}{1+\left(1-\beta_{i}\right) \tau} S_{o_{i}}(\varepsilon) \text { et } \Pi_{o_{i}}(\varepsilon)-\Pi_{v_{i}}=\frac{\left(1-\beta_{i}\right)(1+\tau)}{1+\left(1-\beta_{i}\right) \tau} S_{o_{i}}(\varepsilon) \\
V_{e_{i}}(\varepsilon)-V_{u_{i}}=\frac{\beta_{i}}{1+\left(1-\beta_{i}\right) \tau} S_{e_{i}}(\varepsilon) \text { et } \Pi_{e_{i}}(\varepsilon)-\Pi_{v_{i}}+f_{i}+\tau_{e_{i}}=\frac{\left(1-\beta_{i}\right)(1+\tau)}{1+\left(1-\beta_{i}\right) \tau} S_{e_{i}}(\varepsilon)
\end{gathered}
$$

A présent, en tenant compte des expressions (8) et (9), et en faisant usage des expressions obtenues ci-dessus qui lient les surplus à $V_{o_{i}}(\varepsilon)-V_{u_{i}}$ et à $V_{e_{i}}(\varepsilon)-V_{u_{i}}$ on obtient après quelques manipulations :

$$
\begin{aligned}
& \left(r+\lambda_{i}\right)\left(V_{o_{i}}(\varepsilon)-V_{u_{i}}\right)=w_{o_{i}}(\varepsilon)+\frac{\beta_{i} \lambda_{i}}{1+\tau\left(1-\beta_{i}\right)} \int_{\varepsilon_{d_{i}}}^{\varepsilon_{u_{i}}} S_{e_{i}}(\zeta) d G_{i}(\zeta)-r V_{u_{i}} \\
& \left(r+\lambda_{i}\right)\left(V_{e_{i}}(\varepsilon)-V_{u_{i}}\right)=w_{e_{i}}(\varepsilon)+\frac{\beta_{i} \lambda_{i}}{1+\tau\left(1-\beta_{i}\right)} \int_{\varepsilon_{d_{i}}}^{\varepsilon_{u_{i}}} S_{e_{i}}(\zeta) d G_{i}(\zeta)-r V_{u_{i}}
\end{aligned}
$$

Les expressions des surplus peuvent alors être déterminées en faisant usage des définitions (1) et (2) en utilisant les équations (5), (6), (8) et (9). En imposant $\Pi_{v_{i}}=0$, nous obtenons :

$$
\begin{aligned}
& (r+\lambda) S_{o_{i}}(\varepsilon)=\varepsilon+s_{i}-\tau w_{o_{i}}(\varepsilon)-\lambda_{i}\left(f_{i}+\tau_{e_{i}}\right)+\lambda_{i} \int_{\varepsilon_{d_{i}}}^{\varepsilon_{u_{i}}} S_{e_{i}}(\zeta) d G_{i}(\zeta)-r V_{u_{i}} \\
& (r+\lambda) S_{e_{i}}(\varepsilon)=\varepsilon+s_{i}-\tau w_{e_{i}}(\varepsilon)+r\left(f_{i}+\tau_{e_{i}}\right)+\lambda_{i} \int_{\varepsilon_{d_{i}}}^{\varepsilon_{u_{i}}} S_{e_{i}}(\zeta) d G_{i}(\zeta)-r V_{u_{i}}
\end{aligned}
$$

Comme, $\left(r+\lambda_{i}\right) S_{o_{i}}(\varepsilon)=\left(r+\lambda_{i}\right) \frac{1+\left(1-\beta_{i}\right) \tau}{\beta_{i}}\left(V_{o_{i}}(\varepsilon)-V_{u_{i}}\right)$ d'après $(\mathrm{A}-1)$ tandis que $(r+$ $\left.\lambda_{i}\right) S_{e_{i}}(\varepsilon)=\left(r+\lambda_{i}\right) \frac{1+\left(1-\beta_{i}\right) \tau}{\beta_{i}}\left(V_{e_{i}}(\varepsilon)-V_{u_{i}}\right)$, d'après (A-2), en faisant usage des expressions 
des surplus (A-5), (A-6), et en remplaçant $V_{o_{i}}(\varepsilon)-V_{u_{i}}$ et $V_{e_{i}}(\varepsilon)-V_{u_{i}}$ par leurs expressions données en (A-3) et (A-4), nous obtenons les expressions des salaires suivantes:

$$
\begin{aligned}
& w_{o_{i}}(\varepsilon)=\frac{\beta_{i}}{(1+\tau)}\left[\varepsilon+s_{i}-\lambda_{i}\left(f_{i}+\tau_{e_{i}}\right)\right]+\left(1-\beta_{i}\right) r V_{u_{i}} \\
& w_{e_{i}}(\varepsilon)=\frac{\beta_{i}}{(1+\tau)}\left[\varepsilon+s_{i}+r\left(f_{i}+\tau_{e_{i}}\right)\right]+\left(1-\beta_{i}\right) r V_{u_{i}}
\end{aligned}
$$

Expressions des seuils $\varepsilon_{c_{i}}$ et $\varepsilon_{d_{i}}$ et de la condition de libre entrée. En faisant usage des expressions définissant les surplus (A-5) et (A-6), et en remplaçant les salaires par leurs expressions données en $(\mathrm{A}-7)$ et $(\mathrm{A}-8)$, il vient :

$$
\begin{aligned}
& \left(r+\lambda_{i}\right) S_{o_{i}}(\varepsilon)=\left[\varepsilon+s_{i}-\lambda_{i}\left(f_{i}+\tau_{e_{i}}\right)\right] \frac{1+\tau\left(1-\beta_{i}\right)}{1+\tau}+\lambda_{i} \int_{\varepsilon_{d_{i}}}^{\varepsilon_{u_{i}}} S_{e_{i}}(\zeta) d G_{i}(\zeta)-r V_{u_{i}}\left(1+\tau\left(1-\beta_{i}\right)\right) \\
& \left(r+\lambda_{i}\right) S_{e_{i}}(\varepsilon)=\left[\varepsilon+s_{i}+r\left(f_{i}+\tau_{e_{i}}\right)\right] \frac{1+\tau\left(1-\beta_{i}\right)}{1+\tau}+\lambda_{i} \int_{\varepsilon_{d_{i}}}^{\varepsilon_{u_{i}}} S_{e_{i}}(\zeta) d G_{i}(\zeta)-r V_{u_{i}}\left(1+\tau\left(1-\beta_{i}\right)\right)
\end{aligned}
$$

En évaluant $S_{o_{i}}(\varepsilon)-S_{o_{i}}\left(\varepsilon_{c_{i}}\right)$ et en utilisant la condition (10), il vient :

$$
S_{o_{i}}(\varepsilon)=\frac{\varepsilon-\varepsilon_{c_{i}}}{r+\lambda_{i}} \frac{1+\tau\left(1-\beta_{i}\right)}{1+\tau}
$$

De même, en évaluant $S_{e_{i}}(\varepsilon)-S_{e_{i}}\left(\varepsilon_{d_{i}}\right)$ et à l'aide de la condition (11), il vient :

$$
S_{e_{i}}(\varepsilon)=\frac{\varepsilon-\varepsilon_{d_{i}}}{r+\lambda_{i}} \frac{1+\tau\left(1-\beta_{i}\right)}{1+\tau}
$$

D'autre part, la règle de partage (A-1) implique que l'équation d'actif d'un chômeur (7) s'écrit :

$$
r V_{u_{i}}=b_{i}+\frac{\beta_{i} \theta_{i} m_{i}\left(\theta_{i}\right)}{1+\tau\left(1-\beta_{i}\right)} \int_{\varepsilon_{c_{i}}}^{\varepsilon_{u_{i}}} S_{o_{i}}(\zeta) d G_{i}(\zeta)
$$

ou encore, tenant compte de (A-11) et de (A-1) il vient :

$$
r V_{u_{i}}=b_{i}+\frac{\beta_{i} \theta_{i} m\left(\theta_{i}\right)}{(1+\tau)\left(r+\lambda_{i}\right)} \int_{\varepsilon_{c_{i}}}^{\varepsilon_{u_{i}}}\left(\zeta-\varepsilon_{c_{i}}\right) d G_{i}(\zeta)
$$

En remplaçant cette expression dans celle des surplus (A-9) et (A-10), en tenant compte de (A-12), on aboutit à :

$$
\begin{aligned}
\frac{(1+\tau)\left(r+\lambda_{i}\right)}{1+\tau\left(1-\beta_{i}\right)} S_{o_{i}}(\varepsilon)= & \varepsilon+s_{i}-\lambda_{i}\left(f_{i}+\tau_{e_{i}}\right)-b_{i}(1+\tau) \\
& -\frac{\beta_{i} \theta_{i} m_{i}\left(\theta_{i}\right)}{r+\lambda_{i}} \int_{\varepsilon_{c_{i}}}^{\varepsilon_{u_{i}}}\left(\zeta-\varepsilon_{c_{i}}\right) d G_{i}(\zeta)+\frac{\lambda_{i}}{r+\lambda_{i}} \int_{\varepsilon_{d_{i}}}^{\varepsilon_{u_{i}}}\left(\zeta-\varepsilon_{d_{i}}\right) d G_{i}(\zeta)
\end{aligned}
$$




$$
\begin{aligned}
\frac{(1+\tau)\left(r+\lambda_{i}\right)}{1+\tau\left(1-\beta_{i}\right)} S_{e_{i}}(\varepsilon)= & \varepsilon+s_{i}+r\left(f_{i}+\tau_{e_{i}}\right)-b_{i}(1+\tau) \\
& -\frac{\beta_{i} \theta_{i} m_{i}\left(\theta_{i}\right)}{r+\lambda_{i}} \int_{\varepsilon_{c_{i}}}^{\varepsilon_{u_{i}}}\left(\zeta-\varepsilon_{c_{i}}\right) d G_{i}(\zeta)+\frac{\lambda_{i}}{r+\lambda_{i}} \int_{\varepsilon_{d_{i}}}^{\varepsilon_{u_{i}}}\left(\zeta-\varepsilon_{d_{i}}\right) d G_{i}(\zeta)
\end{aligned}
$$

On aboutit alors aux expressions des seuils de création et de destruction fournies dans le texte en faisant usage de ces expressions et des règles (10) et (11). On a :

$$
\begin{aligned}
0= & \varepsilon_{c_{i}}+s_{i}-\lambda_{i}\left(f_{i}+\tau_{e_{i}}\right)-b_{i}(1+\tau) \\
& -\frac{\beta_{i} \theta_{i} m_{i}\left(\theta_{i}\right)}{r+\lambda_{i}} \int_{\varepsilon_{c_{i}}}^{\varepsilon_{u_{i}}}\left(\zeta-\varepsilon_{c_{i}}\right) d G_{i}(\zeta)+\frac{\lambda_{i}}{r+\lambda_{i}} \int_{\varepsilon_{d_{i}}}^{\varepsilon_{u_{i}}}\left(\zeta-\varepsilon_{d_{i}}\right) d G_{i}(\zeta) \\
0= & \varepsilon_{d_{i}}+s_{i}+r\left(f_{i}+\tau_{e_{i}}\right)-b_{i}(1+\tau) \\
& -\frac{\beta_{i} \theta_{i} m_{i}\left(\theta_{i}\right)}{r+\lambda_{i}} \int_{\varepsilon_{c_{i}}}^{\varepsilon_{u_{i}}}\left(\zeta-\varepsilon_{c_{i}}\right) d G_{i}(\zeta)+\frac{\lambda_{i}}{r+\lambda_{i}} \int_{\varepsilon_{d_{i}}}^{\varepsilon_{u_{i}}}\left(\zeta-\varepsilon_{d_{i}}\right) d G_{i}(\zeta)
\end{aligned}
$$

En soustrayant ces deux expressions, on obtient la relation suivante entre $\varepsilon_{d_{i}}$ et $\varepsilon_{c_{i}}$ :

$$
\varepsilon_{c_{i}}=\varepsilon_{d_{i}}+\left(r+\lambda_{i}\right)\left(f_{i}+\tau_{e_{i}}\right)
$$

Libre entrée. La libre entrée implique $\Pi_{v_{i}}=0$. Tenant compte de l'expression de $\Pi_{v_{i}}$ fournie par (4) et de la règle de partage $(\mathrm{A}-1) \Pi_{o_{i}}(\varepsilon)=\frac{\left(1-\beta_{i}\right)(1+\tau)}{1+\left(1-\beta_{i}\right) \tau} S_{o_{i}}(\varepsilon)$, nous avons :

$$
\frac{c_{i}}{m_{i}\left(\theta_{i}\right)}=\frac{\left(1-\beta_{i}\right)(1+\tau)}{1+\left(1-\beta_{i}\right) \tau} \int_{\varepsilon_{c_{i}}}^{\varepsilon_{u_{i}}} S_{o_{i}}(\zeta) d G_{i}(\zeta)
$$

ou encore, en tenant compte de l'expression du surplus par son expression dans (A-11) :

$$
\frac{c_{i}}{m_{i}\left(\theta_{i}\right)}=\frac{1-\beta_{i}}{r+\lambda_{i}} \int_{\varepsilon_{c_{i}}}^{\varepsilon_{u_{i}}}\left(\zeta-\varepsilon_{c_{i}}\right) d G_{i}(\zeta)
$$

Il est également possible de réécrire cette dernière expression en faisant apparaître les paramètres de politiques économiques. A l'aide de la relation (A-19), il vient :

$$
\frac{c_{i}}{m_{i}\left(\theta_{i}\right)}=\left(1-\beta_{i}\right)\left[\int_{\varepsilon_{c_{i}}}^{\varepsilon_{u_{i}}} \frac{\zeta-\varepsilon_{d_{i}}}{r+\lambda_{i}}-f_{i}-\tau_{e_{i}}\right] d G_{i}(\zeta)
$$

\section{Annexe 5.2 Propriétés des seuils de création, destruction et de l'équation de libre entrée}

Propriétés des seuils de création et de destruction. En tenant compte de la relation (A-19), le seuil de création défini en (A-17) peut se réécrire comme :

$$
\begin{aligned}
0= & \varepsilon_{c_{i}}+s_{i}-\lambda_{i}\left(f_{i}+\tau_{e_{i}}\right)-b_{i}(1+\tau)-\frac{\beta_{i} \theta_{i} m_{i}\left(\theta_{i}\right)}{r+\lambda_{i}} \int_{\varepsilon_{c_{i}}}^{\varepsilon_{u_{i}}}\left(\zeta-\varepsilon_{c_{i}}\right) d G_{i}(\zeta) \\
& +\frac{\lambda_{i}}{r+\lambda_{i}} \int_{\varepsilon_{c_{i}}-\left(r+\lambda_{i}\right)\left(f_{i}+\tau_{e_{i}}\right)}^{\varepsilon_{u_{i}}}\left(\zeta-\varepsilon_{c_{i}}+\left(r+\lambda_{i}\right)\left(f_{i}+\tau_{e_{i}}\right)\right) d G_{i}(\zeta)
\end{aligned}
$$


Les propriétés d'équilibre partiel du seuil de création $\varepsilon_{c_{i}}$ découlent de l'application du théorème des fonctions implicites à cette expression. En notant $\eta_{i} \in[0 ; 1]$, l'élasticité de la fonction d'appariement par rapport au taux de chômage, il vient :

$$
\begin{gathered}
\frac{d \varepsilon_{c_{i}}}{d s_{i}}=-\frac{r+\lambda_{i}}{r+\beta_{i} \theta_{i} m_{i}\left(\theta_{i}\right)\left(1-G_{i}\left(\varepsilon_{c_{i}}\right)\right)+\lambda_{i} G_{i}\left(\varepsilon_{d_{i}}\right)}<0 ; \quad \frac{d \varepsilon_{c_{i}}}{d \theta_{i}}=\frac{\beta_{i} m_{i}\left(\theta_{i}\right)\left[1-\eta_{i}\left(\theta_{i}\right)\right] \int_{\varepsilon_{i}}^{\varepsilon_{u_{i}}}\left(\zeta-\varepsilon_{c_{i}}\right) d G_{i}(\zeta)}{r+\beta_{i} \theta_{i} m_{i}\left(\theta_{i}\right)\left(1-G_{i}\left(\varepsilon_{c_{i}}\right)\right)+\lambda_{i} G_{i}\left(\varepsilon_{d_{i}}\right)}>0 \\
\frac{d \varepsilon_{c_{i}}}{d b_{i}}=\frac{\left(r+\lambda_{i}\right)(1+\tau)}{r+\beta_{i} \theta_{i} m_{i}\left(\theta_{i}\right)\left(1-G_{i}\left(\varepsilon_{c_{i}}\right)\right)+\lambda_{i} G_{i}\left(\varepsilon_{d_{i}}\right)}>0 ; \quad \frac{d \varepsilon_{c_{i}}}{d \tau}=\frac{\left(r+\lambda_{i}\right.}{r+\beta_{i} \theta_{i} m_{i}\left(\theta_{i}\right)\left(1-G_{i}\left(\varepsilon_{c_{i}}\right)\right)+\lambda_{i} G_{i}\left(\varepsilon_{d_{i}}\right)}>0 \\
\frac{d \varepsilon_{c_{i}}}{d f_{i}}=\frac{d \varepsilon_{c_{i}}}{d \tau e_{i}}=\frac{\left(r+\lambda_{i}\right) \lambda_{i} G_{i}\left(\varepsilon_{d_{i}}\right)}{r+\beta_{i} \theta_{i} m_{i}\left(\theta_{i}\right)\left(1-G_{i}\left(\varepsilon_{c_{i}}\right)\right)+\lambda_{i} G_{i}\left(\varepsilon_{d_{i}}\right)}>0 ; \quad \frac{d \varepsilon_{c_{i}}}{d \beta_{i}}=\frac{\theta_{i} m_{i}\left(\theta_{i}\right) \int_{\varepsilon_{i}}^{\varepsilon_{c_{i}}}\left(\zeta-\varepsilon_{c_{i}}\right) d G_{i}(\zeta)}{r+\beta_{i} \theta_{i} m_{i}\left(\theta_{i}\right)\left(1-G_{i}\left(\varepsilon_{c_{i}}\right)\right)+\lambda_{i} G_{i}\left(\varepsilon_{d_{i}}\right)}>0
\end{gathered}
$$

Finalement, en utilisant l'expression définissant, $\varepsilon_{d_{i}}=\varepsilon_{c_{i}}-\left(r+\lambda_{i}\right)\left(f_{i}+\tau_{e_{i}}\right)$, les propriétés d'équilibre partiel du seuil de destruction $\varepsilon_{d_{i}}$ vérifient :

$$
\begin{array}{cc}
\frac{d \varepsilon_{d_{i}}}{d s_{i}}=\frac{d \varepsilon_{c_{i}}}{d s_{i}}<0 ; & \frac{d \varepsilon_{d_{i}}}{d \theta_{i}}=\frac{d \varepsilon_{c_{i}}}{d \theta_{i}}>0 \\
\frac{d \varepsilon_{d_{i}}}{d f_{i}}=\frac{d \varepsilon_{c_{i}}}{d f_{i}}-(r+\lambda)<0 ; & \frac{d \varepsilon_{d_{i}}}{d \tau_{e_{i}}}=\frac{d \varepsilon_{c_{i}}}{d \tau_{e_{i}}}-\left(r+\lambda_{i}\right)<0 \\
\frac{d \varepsilon_{d_{i}}}{d b_{i}}=\frac{d \varepsilon_{c_{i}}}{d b_{i}}>0 ; & \frac{d \varepsilon_{d_{i}}}{d \tau}=\frac{d \varepsilon_{c_{i}}}{d \tau}>0 \\
\frac{d \varepsilon_{d_{i}}}{d \beta_{i}}=\frac{d \varepsilon_{c_{i}}}{d \beta_{i}}>0 ; &
\end{array}
$$

Existence et unicité de l'équilibre à taxation donnée. En notant que $\varepsilon_{d_{i}}=\varepsilon_{c_{i}}-$ $(r+\lambda)\left(f_{i}+\tau_{e_{i}}\right)$, un équilibre à taxation donnée se ramène à l'étude des deux équations :

$$
\begin{gathered}
0=\varepsilon_{c_{i}}+s_{i}-\lambda\left(f_{i}+\tau_{e_{i}}\right)-b_{i}(1+\tau)-\frac{\beta_{i} \theta_{i} m_{i}\left(\theta_{i}\right)}{r+\lambda_{i}} \int_{\varepsilon_{c_{i}}}^{\varepsilon_{u_{i}}}\left(\zeta-\varepsilon_{c_{i}}\right) d G_{i}(\zeta) \\
+\frac{\lambda_{i}}{r+\lambda_{i}} \int_{\varepsilon_{c_{i}}-\left(r+\lambda_{i}\right)\left(f_{i}+\tau_{e_{i}}\right)}^{\varepsilon_{u_{i}}}\left(\zeta-\varepsilon_{c_{i}}+\left(r+\lambda_{i}\right)\left(f_{i}+\tau_{e_{i}}\right)\right) d G_{i}(\zeta) \\
0=\frac{c_{i}}{m_{i}\left(\theta_{i}\right)}-\frac{1-\beta_{i}}{r+\lambda_{i}} \int_{\varepsilon_{c_{i}}}^{\varepsilon_{u_{i}}}\left(\zeta-\varepsilon_{c_{i}}\right) d G_{i}(\zeta)
\end{gathered}
$$

A partir de la première équation, on peut définir $\varepsilon_{c_{i}}\left(\theta_{i}\right)$ solution de (A-24). Cette fonction est définie de $\left[0,+\infty\left[\right.\right.$ vers $\left[0,+\infty\left[\right.\right.$. Elle est continue et croissante, avec $\lim _{\theta_{i} \rightarrow 0} \varepsilon_{c_{i}}\left(\theta_{i}\right)=\underline{\varepsilon_{c_{i}}}$ tel que

$$
\begin{aligned}
0= & \frac{\varepsilon_{c_{i}}}{}+s_{i}-\lambda_{i}\left(f_{i}+\tau_{e_{i}}\right)-b_{i}(1+\tau) \\
& +\frac{\lambda_{i}}{r+\lambda_{i}} \int_{\underline{\varepsilon_{c_{i}}}-\left(r+\lambda_{i}\right)\left(f_{i}+\tau_{e_{i}}\right)}^{\varepsilon_{u_{i}}}\left(\zeta-\underline{\varepsilon_{c_{i}}}+\left(r+\lambda_{i}\right)\left(f_{i}+\tau_{e_{i}}\right)\right) d G_{i}(\zeta)
\end{aligned}
$$

On peut alors définir $\Psi\left(\theta_{i}\right)$ telle que :

$$
\Psi\left(\theta_{i}\right)=\frac{c_{i}}{m_{i}\left(\theta_{i}\right)}-\frac{1-\beta_{i}}{r+\lambda_{i}} \int_{\varepsilon_{c_{i}}\left(\theta_{i}\right)}^{\varepsilon_{u_{i}}}\left(\zeta-\varepsilon_{c_{i}}\left(\theta_{i}\right)\right) d G(\zeta)
$$

Résoudre un équilibre revient à déterminer $\overline{\theta_{i}}$ solution de $\Psi\left(\overline{\theta_{i}}\right)=0$. Puisque $\lim _{\theta_{i} \rightarrow+\infty} \frac{c_{i}}{m_{i}\left(\theta_{i}\right)}=$ $+\infty$ nous avons $\lim _{\theta_{i} \rightarrow+\infty} \Psi\left(\theta_{i}\right)=+\infty$. Comme $\lim _{\theta_{i} \rightarrow 0} \frac{c_{i}}{m_{i}\left(\theta_{i}\right)}=0$, on peut établir par continuité qu'il existe un équilibre (non trivial) lorsque

$$
\lim _{\theta_{i} \rightarrow 0} \Psi\left(\theta_{i}\right)=-\frac{1-\beta_{i}}{r+\lambda_{i}} \int_{\varepsilon_{c_{i}}\left(\theta_{i}\right)}^{\varepsilon_{u_{i}}}\left(\zeta-\varepsilon_{c_{i}}\left(\theta_{i}\right)\right) d G_{i}(\zeta)<0
$$


ce qui n'est pas garanti. En effet, considérons (A-24). Il est facile d'établir que pour certaines valeurs de $s_{i}$, on peut avoir $\lim _{\theta_{i} \rightarrow 0} \varepsilon_{c_{i}}\left(\theta_{i}\right) \geq \varepsilon_{u}$ de sorte que

$$
\lim _{\theta_{i} \rightarrow 0} \Psi\left(\theta_{i}\right)=-\frac{1-\beta_{i}}{r+\lambda_{i}} \int_{\varepsilon_{c_{i}}\left(\theta_{i}\right)}^{\varepsilon_{u_{i}}}\left(\zeta-\varepsilon_{c_{i}}\left(\theta_{i}\right)\right) d G_{i}(\zeta)>0
$$

On définit alors $\underline{s}$ comme le seuil de productivité minimal requis pour avoir $\lim _{\theta_{i} \rightarrow 0} \varepsilon_{c_{i}}(\theta) \leq \varepsilon_{u}$. A l'aide de (A-24), ce seuil s'écrit :

$$
\begin{aligned}
\underline{s}= & -\varepsilon_{u}+\lambda_{i}\left(f_{i}+\tau_{e_{i}}\right)+b_{i}(1+\tau) \\
& -\frac{\lambda_{i}}{r+\lambda_{i}} \int_{\varepsilon_{u}-\left(r+\lambda_{i}\right)\left(f_{i}+\tau_{e_{i}}\right)}^{\varepsilon_{u_{i}}}\left(\zeta-\varepsilon_{u_{i}}+\left(r+\lambda_{i}\right)\left(f_{i}+\tau_{e_{i}}\right)\right) d G_{i}(\zeta)
\end{aligned}
$$

Après intégration par partie, on aboutit à l'expression fournie dans le texte. Les propriétés de $\underline{s}$ découlent de l'application du théorème des fonctions implicites à cette expression. Considérons à présent le cas où $s_{2}>\underline{s}$ de sorte que l'existence d'un équilibre est assurée pour chacun des deux marchés. La dérivée $\Psi^{\prime}\left(\theta_{i}\right)$ tirée de (A-26) s'écrit :

$$
\Psi^{\prime}\left(\theta_{i}\right)=-\frac{c_{i} m_{i}^{\prime}\left(\theta_{i}\right)}{m_{i}\left(\theta_{i}\right)^{2}}+\frac{1-\beta_{i}}{r+\lambda_{i}}\left[\varepsilon_{c_{i}}^{\prime}\left(\theta_{i}\right)\left[1-G\left(\varepsilon_{c_{i}}\left(\theta_{i}\right)\right)\right]\right]
$$

Elle est positive puisque $m_{i}^{\prime}\left(\theta_{i}\right)<0$ et $\varepsilon_{c_{i}}^{\prime}\left(\theta_{i}\right)>0$. Par suite, lorsqu'il existe une valeur de $\overline{\theta_{i}}$ solution de l'équation de libre entrée, celle-ci est unique.

Statique comparative de l'équilibre. Considérons le système formé par les équations (A-24) et (A-25). On note $J_{i}$ la matrice jacobienne de ce système. Il vient :

$$
J_{i}=\left(\begin{array}{cc}
\frac{r+\beta_{i} \theta_{i} m_{i}\left(\theta_{i}\right)\left(1-G\left(\varepsilon_{c_{i}}\right)\right)+\lambda_{i} G_{i}\left(\varepsilon_{d_{i}}\right)}{r+\lambda_{i}} & \frac{-\beta_{i} m_{i}\left(\theta_{i}\right)\left(1-\eta_{i}\left(\theta_{i}\right)\right) \int_{\varepsilon_{i}}^{\varepsilon_{u_{i}}}\left(x-\varepsilon_{c_{i}}\right) d G_{i}(x)}{r+\lambda_{i}} \\
\frac{1-\beta_{i}}{r+\lambda_{i}}\left(1-G_{i}\left(\varepsilon_{c_{i}}\right)\right) & \frac{-m_{i}^{\prime}\left(\theta_{i}\right)}{m_{i}\left(\theta_{i}\right)^{2}}
\end{array}\right)
$$

Il est immédiat de remarquer que le déterminant de cette matrice est positif, i.e. $\operatorname{det} J_{i}>0$. Par suite, il est aisé d'établir que :

$$
\begin{gathered}
\left(\begin{array}{c}
\frac{d \theta_{i}}{d s_{i}} \\
\frac{d \varepsilon_{i}}{d s_{i}}
\end{array}\right)=\frac{1}{\operatorname{det} J_{i}}\left(\begin{array}{c}
\frac{1-\beta_{i}}{r+\lambda_{i}}\left(1-G_{i}\left(\varepsilon_{c_{i}}\right)\right) \\
\frac{c_{i} m_{i}^{\prime}\left(\theta_{i}\right)}{m_{i}\left(\theta_{i}\right)^{2}}
\end{array}\right) \\
\left(\begin{array}{c}
\frac{d \theta_{i}}{d f_{i}} \\
\frac{d \varepsilon_{i}}{d f_{i}}
\end{array}\right)=\left(\begin{array}{c}
\frac{d \theta_{i}}{d \tau_{e_{i}}} \\
\frac{d c_{i}}{d \tau_{e_{i}}}
\end{array}\right)=\frac{-1}{\operatorname{det} J_{i}}\left(\begin{array}{c}
\frac{1-\beta_{i}}{r+\lambda_{i}}\left(1-G\left(\varepsilon_{c_{i}}\right)\right) \lambda_{i} G_{i}\left(\varepsilon_{d_{i}}\right) \\
\frac{c_{i} m_{i}^{\prime}\left(\theta_{i}\right)}{m_{i}\left(\theta_{i}\right)^{2}} \lambda_{i} G_{i}\left(\varepsilon_{d_{i}}\right)
\end{array}\right) \\
\left(\begin{array}{c}
\frac{d \theta_{i}}{d b_{i}} \\
\frac{d \varepsilon_{i}}{d b_{i}}
\end{array}\right)=\frac{-(1+\tau)}{\operatorname{det} J_{i}}\left(\begin{array}{c}
\frac{1-\beta_{i}}{r+\lambda_{i}}\left(1-G\left(\varepsilon_{c_{i}}\right)\right) \\
\frac{c_{i} m_{i}^{\prime}\left(\theta_{i}\right)}{m_{i}\left(\theta_{i}\right)^{2}}
\end{array}\right) \\
\left(\begin{array}{c}
\frac{d \theta_{i}}{d \tau} \\
\frac{d \varepsilon_{c_{i}}}{d \tau}
\end{array}\right)=\frac{-b_{i}}{\operatorname{det} J_{i}}\left(\begin{array}{c}
\frac{1-\beta_{i}}{r+\lambda_{i}}\left(1-G_{i}\left(\varepsilon_{c_{i}}\right)\right) \\
\frac{c_{i} m_{i}^{\prime}\left(\theta_{i}\right)}{m_{i}\left(\theta_{i}\right)^{2}}
\end{array}\right)
\end{gathered}
$$




$$
\left(\begin{array}{c}
\frac{d \theta_{i}}{d \beta_{i}} \\
\frac{d \varepsilon_{c_{i}}}{d \beta_{i}}
\end{array}\right)=\frac{-\int_{\varepsilon_{c_{i}}}^{\varepsilon_{u_{i}}} \frac{\left(x-\varepsilon_{c_{i}}\right)}{r+\lambda_{i}} d G_{i}(x)}{\operatorname{det} J_{i}}\left(\begin{array}{c}
\frac{r+\theta_{i} m_{i}\left(\theta_{i}\right)\left(1-G\left(\varepsilon_{c_{i}}\right)\right)+\lambda_{i} G_{i}\left(\varepsilon_{d_{i}}\right)}{\left(r+\lambda_{i}\right)} \\
\left(\beta_{i}-\eta_{i}\right) m\left(\theta_{i}\right) \int_{\varepsilon_{c_{i}}}^{\varepsilon_{u_{i}}} \frac{\left(x-\varepsilon_{c_{i}}\right) d G_{i}(x)}{r+\lambda_{i}}
\end{array}\right)
$$

Nous obtenons ainsi les propriétés suivantes :

$$
\begin{aligned}
& \frac{d \theta_{i}}{d s_{i}}>0 ; \quad \frac{d \theta_{i}}{d f_{i}}<0 ; \quad \frac{d \theta_{i}}{d \tau_{e_{i}}}<0 ; \quad \frac{d \theta_{i}}{d b_{i}}<0 ; \quad \frac{d \theta_{i}}{d \tau}<0 ; \quad \frac{d \theta_{i}}{d \beta_{i}}<0 \\
& \frac{d \varepsilon_{c_{i}}}{d s_{i}}<0 ; \quad \frac{d \varepsilon_{c_{i}}}{d f_{i}}>0 ; \quad \frac{d \varepsilon_{c_{i}}}{d \tau_{e_{i}}}>0 ; \quad \frac{d \varepsilon_{c_{i}}}{d b_{i}}>0 ; \quad \frac{d \varepsilon_{c_{i}}}{d \tau}>0 ; \quad \frac{d \varepsilon_{c_{i}}}{d \beta_{i}} \text { du signe de } \eta_{i}-\beta_{i}
\end{aligned}
$$

Par suite, en utilisant la définition de $\varepsilon_{d_{i}}$, il vient :

$$
\frac{d \varepsilon_{d_{i}}}{d s_{i}}<0 ; \quad \frac{d \varepsilon_{d_{i}}}{d f_{i}}<0 ; \quad \frac{d \varepsilon_{d_{i}}}{d \tau_{e_{i}}}<0 ; \quad \frac{d \varepsilon_{d_{i}}}{d b_{i}}>0 ; \quad \frac{d \varepsilon_{d_{i}}}{d \tau}>0 ; \quad \frac{d \varepsilon_{d_{i}}}{d \beta_{i}} \text { du signe de } \eta_{i}-\beta_{i}
$$

\section{Annexe 5.3 Calculs intermédiaires : production moyenne}

$\mathrm{Au}$ sein de chaque secteur, la production totale évolue selon :

$$
\begin{aligned}
d Y_{i} / d t= & \mu_{i} U_{i} \int_{\varepsilon_{c_{i}}}^{\varepsilon_{u_{i}}}\left(s_{i}+\varepsilon\right) \frac{d G_{i}(\varepsilon)}{1-G_{i}\left(\varepsilon_{c_{i}}\right)} \\
& +\lambda_{i}\left(1-G_{i}\left(\varepsilon_{d_{i}}\right)\right)\left(P_{i}-U_{i}\right) \int_{\varepsilon_{d_{i}}}^{\varepsilon_{u_{i}}}\left(s_{i}+\varepsilon\right) \frac{d G_{i}(\varepsilon)}{1-G_{i}\left(\varepsilon_{d_{i}}\right)}-\left(\delta+\lambda_{i}\right) Y_{i}
\end{aligned}
$$

De sorte qu'à l'état stationnaire :

$$
\begin{aligned}
Y_{i}= & \frac{\mu_{i}}{\delta+\lambda_{i}} U_{i} \int_{\varepsilon_{c_{i}}}^{\varepsilon_{u_{i}}}\left(s_{i}+\varepsilon\right) \frac{d G_{i}(\varepsilon)}{1-G_{i}\left(\varepsilon_{c_{i}}\right)} \\
& +\frac{\lambda_{i}\left(1-G_{i}\left(\varepsilon_{d_{i}}\right)\right)}{\delta+\lambda_{i}}\left(P_{i}-U_{i}\right) \int_{\varepsilon_{d_{i}}}^{\varepsilon_{u_{i}}}\left(s_{i}+\varepsilon\right) \frac{d G_{i}(\varepsilon)}{1-G_{i}\left(\varepsilon_{d_{i}}\right)} \\
= & \left(1-u_{i}\right) P_{i}\left[s_{i}+\frac{\left[\delta+\lambda_{i} G_{i}\left(\varepsilon_{d_{i}}\right)\right] \int_{\varepsilon_{c_{i}}}^{\varepsilon_{u_{i}}} \varepsilon \frac{d G_{i}(\varepsilon)}{1-G_{i}\left(\varepsilon_{c_{i}}\right)}+\lambda_{i}\left(1-G_{i}\left(\varepsilon_{d_{i}}\right)\right) \int_{\varepsilon_{d_{i}}}^{\varepsilon_{u_{i}}} \varepsilon \frac{d G_{i}(\varepsilon)}{1-G_{i}\left(\varepsilon_{d_{i}}\right)}}{\delta+\lambda_{i}}\right]
\end{aligned}
$$

On en déduit l'expression de la production moyenne réalisée par chaque emploi au sein du secteur $i, \bar{y}_{i}=Y_{i} /\left[\left(1-u_{i}\right) P_{i}\right]$.

$$
\bar{y}_{i}=\left[s_{i}+\frac{n_{o_{i}}}{n_{o_{i}}+n_{e_{i}}} \int_{\varepsilon_{c_{i}}}^{\varepsilon_{u_{i}}} \varepsilon \frac{d G_{i}(\varepsilon)}{1-G_{i}\left(\varepsilon_{c_{i}}\right)}+\frac{n_{e_{i}}}{n_{o_{i}}+n_{e_{i}}} \int_{\varepsilon_{d_{i}}}^{\varepsilon_{u_{i}}} \varepsilon \frac{d G_{i}(\varepsilon)}{1-G_{i}\left(\varepsilon_{d_{i}}\right)}\right]
$$

où $\frac{n_{o_{i}}}{n_{o_{i}}+n_{e_{i}}}=\frac{\delta+\lambda_{i} G_{i}\left(\varepsilon_{d_{i}}\right)}{\delta+\lambda_{i}}$ et $\frac{n_{e_{i}}}{n_{o_{i}}+n_{e_{i}}}=\frac{\lambda_{i}\left(1-G_{i}\left(\varepsilon_{d_{i}}\right)\right)}{\delta+\lambda_{i}}$ d'après (19).

Par suite, la productivité moyenne des emplois de l'économie s'écrit : $\bar{y}=\frac{\Sigma_{i}\left(1-u_{i}\right) P_{i} \bar{y}_{i}}{\Sigma_{i}\left(1-u_{i}\right) P_{i}}$ et correspond à l'expression figurant dans le texte. 


\section{Annexe 5.4 Propriétés du rendement de l'éducation}

Nous avons $r V_{u_{i}}=b_{i}+\frac{\beta_{i} \theta_{i} m\left(\theta_{i}\right)}{(1+\tau)\left(r+\lambda_{i}\right)} \int_{\varepsilon_{c_{i}}}^{\varepsilon_{u_{i}}}\left(\zeta-\varepsilon_{c_{i}}\right) d G_{i}(\zeta)$ d'après (A-14). A taux de taxe donné, il vient :

$$
\begin{aligned}
& \frac{d V_{u_{i}}}{d f_{i}}=\frac{d V_{u_{i}}}{d \theta_{i}} \frac{d \theta_{i}}{d f_{i}}+\frac{d V_{u_{i}}}{d \varepsilon_{c_{i}}} \frac{d \varepsilon_{c_{i}}}{d f_{i}} \leq 0 \\
& \frac{d V_{u_{i}}}{d \tau_{e_{i}}}=\frac{d V_{u_{i}}}{d \theta_{i}} \frac{d \theta_{i}}{d \tau_{e_{i}}}+\frac{d V_{u_{i}}}{d \varepsilon_{c_{i}}} \frac{d \varepsilon_{c_{i}}}{d \tau_{e_{i}}} \leq 0 \\
& \frac{d V_{u_{i}}}{d \tau}=\frac{\partial V_{u_{i}}}{\partial \tau}+\frac{\partial V_{u_{i}}}{\partial \theta_{i}} \frac{d \theta_{i}}{d \tau}+\frac{\partial V_{u_{i}}}{\partial \varepsilon_{c_{i}}} \frac{d \varepsilon_{c_{i}}}{d \tau} \leq 0
\end{aligned}
$$

Comme $P_{1}=\Phi(\widehat{C})=\Phi\left(r V_{u_{1}}-r V_{u_{2}}\right)$, on en déduit :

$$
\begin{aligned}
& \frac{d P_{1}}{d f_{1}}=\Phi^{\prime}(\widehat{C}) r \frac{d V_{u_{1}}}{d f_{1}} ; \frac{d P}{d f_{2}}=-\Phi^{\prime}(\widehat{C}) r \frac{d V_{u_{2}}}{d f_{2}} ; \\
& (-) \quad(+) \\
& \frac{d P_{1}}{d \tau_{e_{1}}}=\Phi^{\prime}(\widehat{C}) r \frac{d V_{u_{1}}}{d \tau_{e_{1}}} ; \frac{d P}{d \tau_{e_{2}}}=-\Phi^{\prime}(\widehat{C}) r \frac{d V_{u_{2}}}{d \tau_{e_{2}}} ;
\end{aligned}
$$

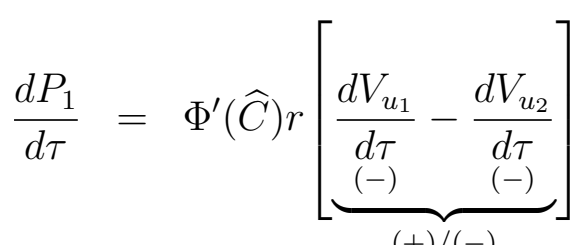

Ce qui correspond aux propriétés de statique comparative qui figurent dans le texte. 


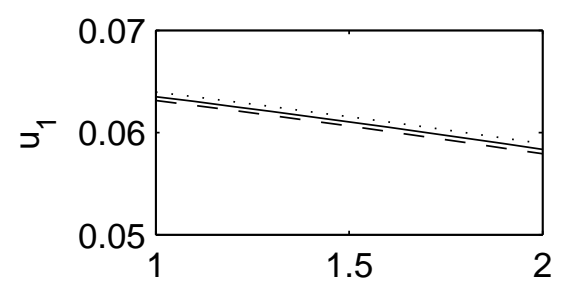

fig $1 \mathrm{a}$

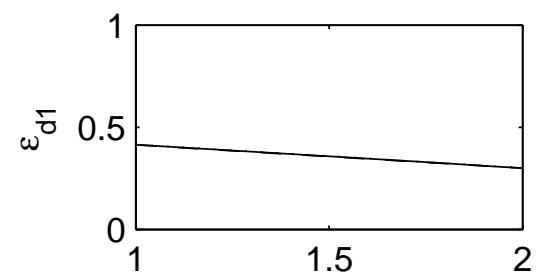

fig $1 d$

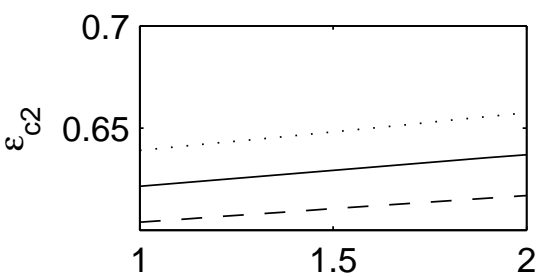

fig $1 \mathrm{~g}$

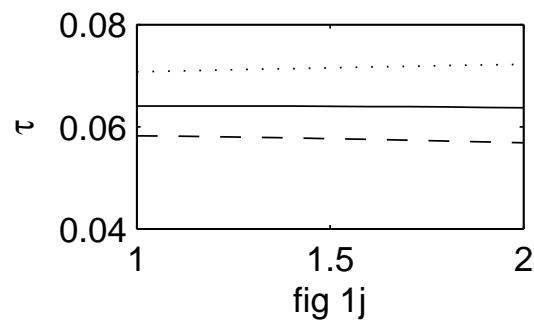

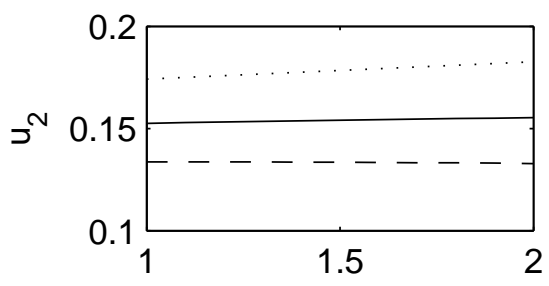

fig $1 \mathrm{~b}$

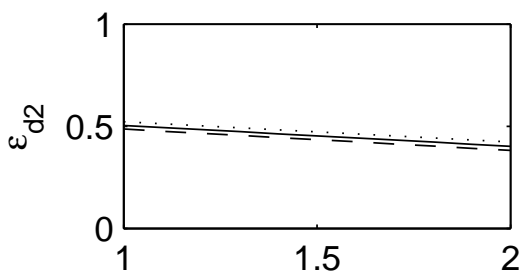

fig $1 \mathrm{e}$

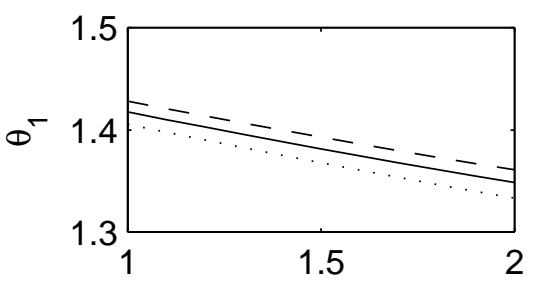

fig $1 \mathrm{~h}$

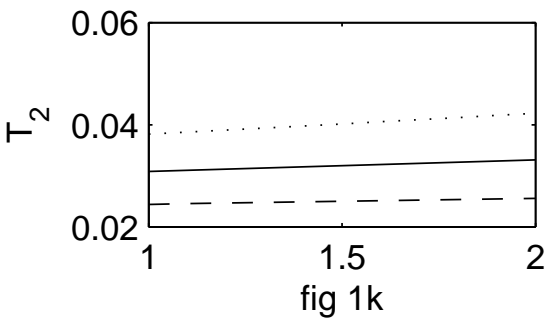

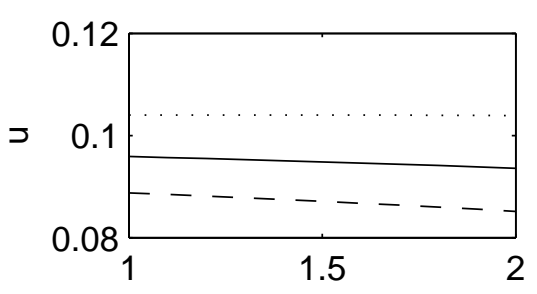

fig $1 \mathrm{c}$

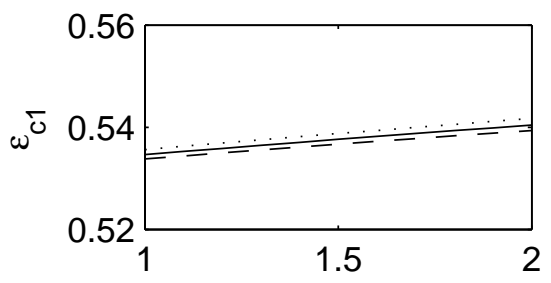

fig $1 f$

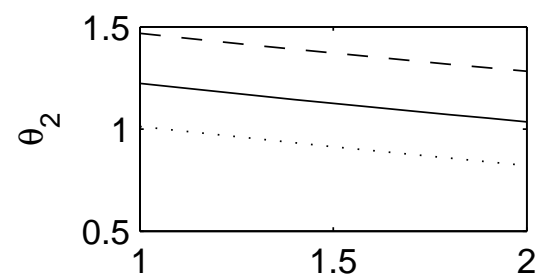

fig $1 \mathrm{i}$

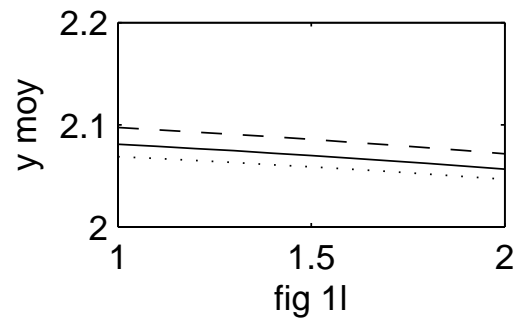

Figure 1 - Effets d'une hausse de la rigueur de la LPE, $\gamma$, sur le niveau et la composition du chômage lorsque $s_{2}$ est à sa valeur moyenne (trait continu), est faible (traits pointillés), est élevé (traits discontinus longs). 

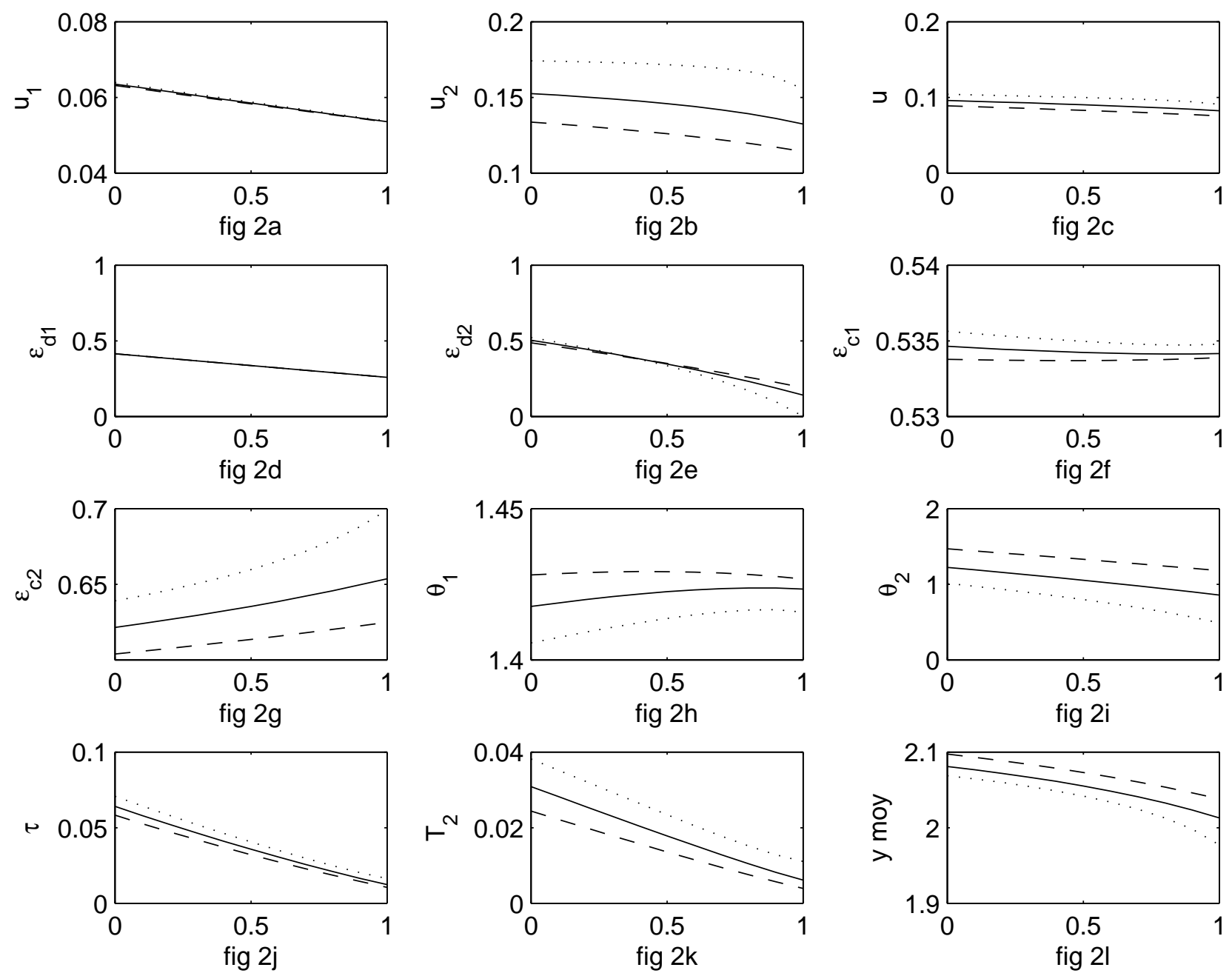

FIGURE 2 - Effets d'une hausse de l'indice de modulation, $e$, sur le niveau et la composition du chômage lorsque $s_{2}$ est à sa valeur moyenne (trait continu), est faible (traits pointillés), est élevé (traits discontinus longs). 

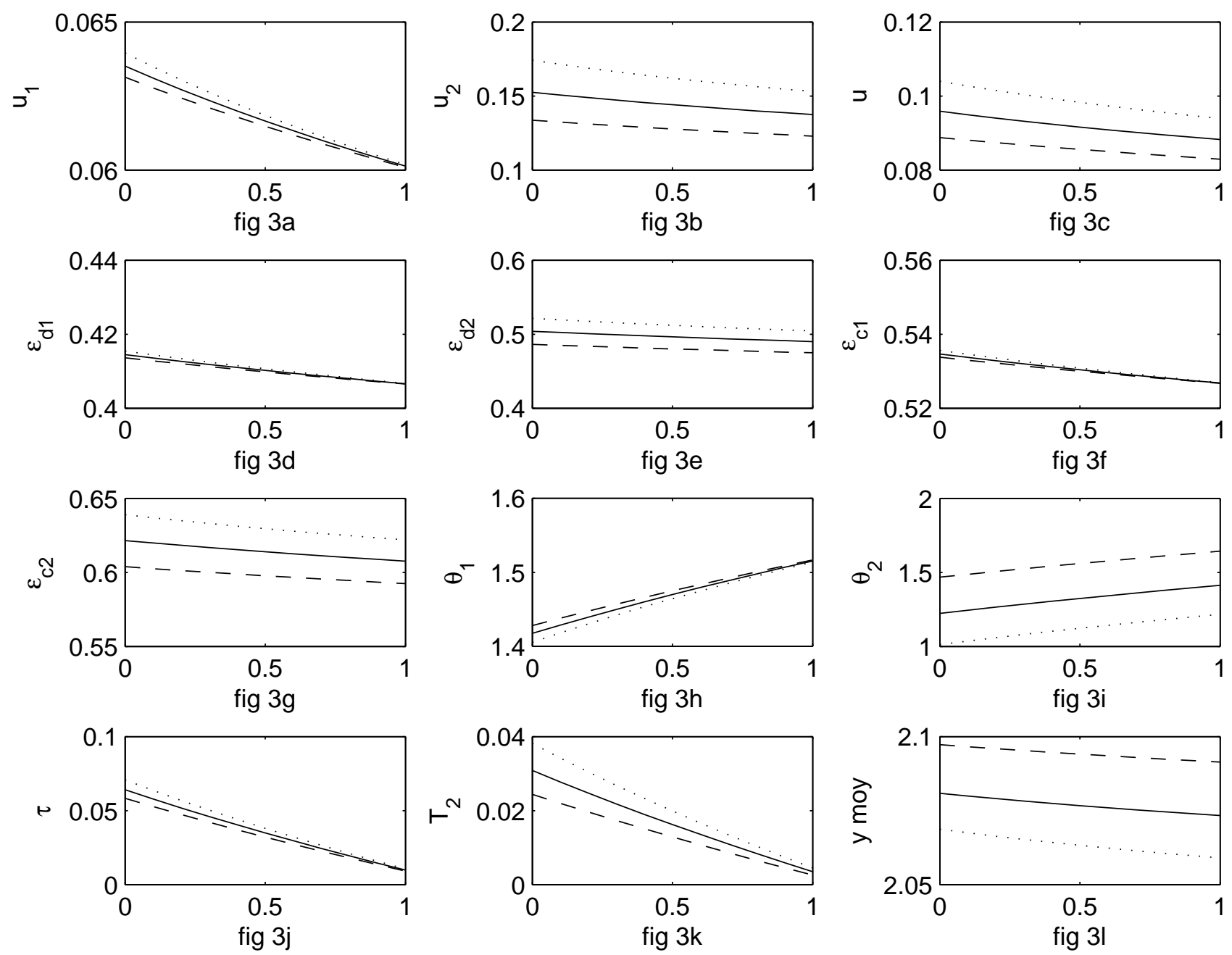

FiguRE 3 - Effets d'une réforme de la LPE sur le niveau et la composition du chômage lorsque $s_{2}$ est à sa valeur moyenne (trait continu), est faible (traits pointillés), est élevé (traits discontinus longs). 

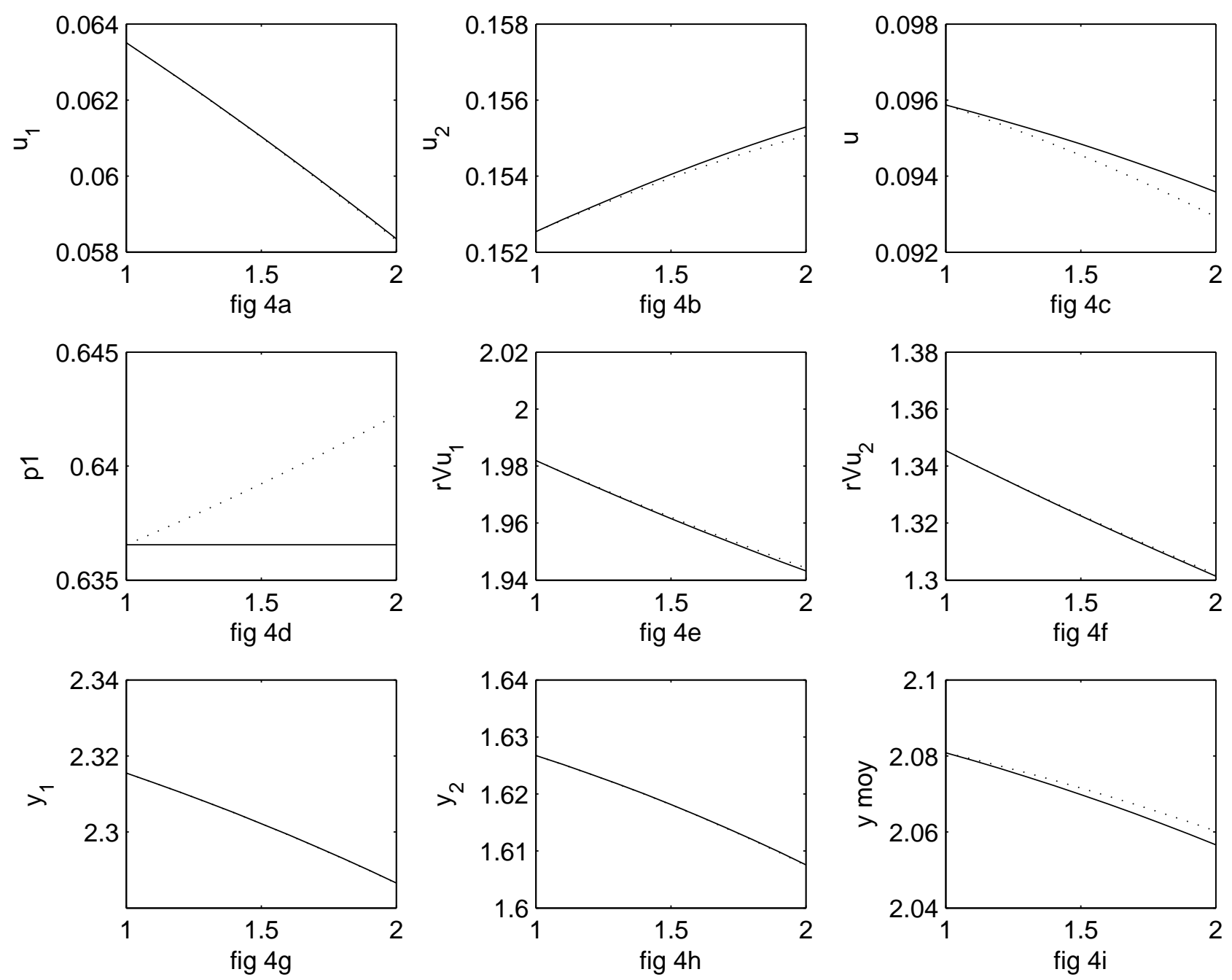

Figure 4 - Effets d'une hausse de la rigueur de la LPE, $\gamma$, lorsque l'education est exogène (trait continu) et lorsque l'éducation est endogène (traits pointillés). 

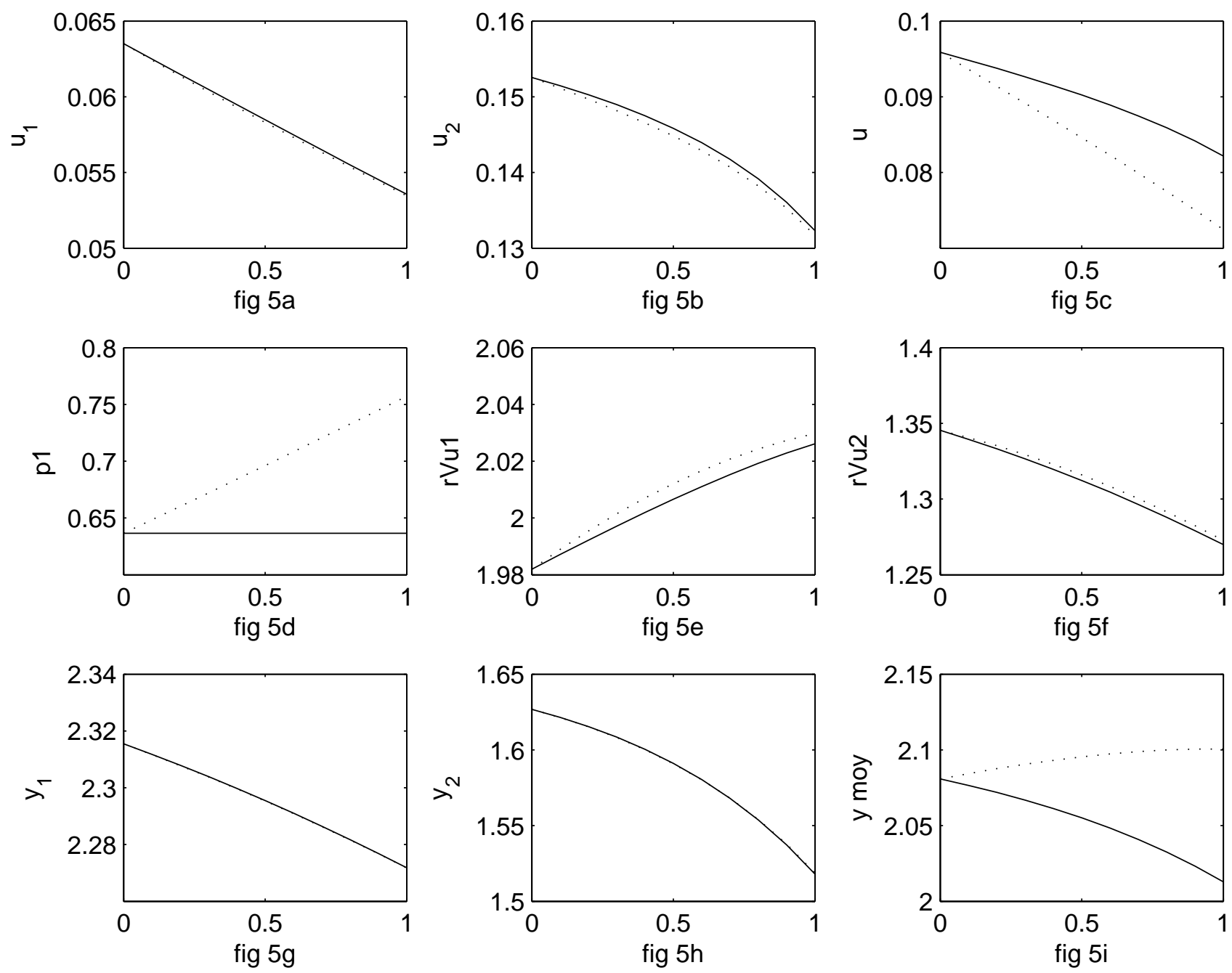

Figure 5 - Effets d'une hausse de l'indice de modulation, $e$, lorsque l'education est exogène (trait continu) et lorsque l'éducation est endogène (traits pointillés). 

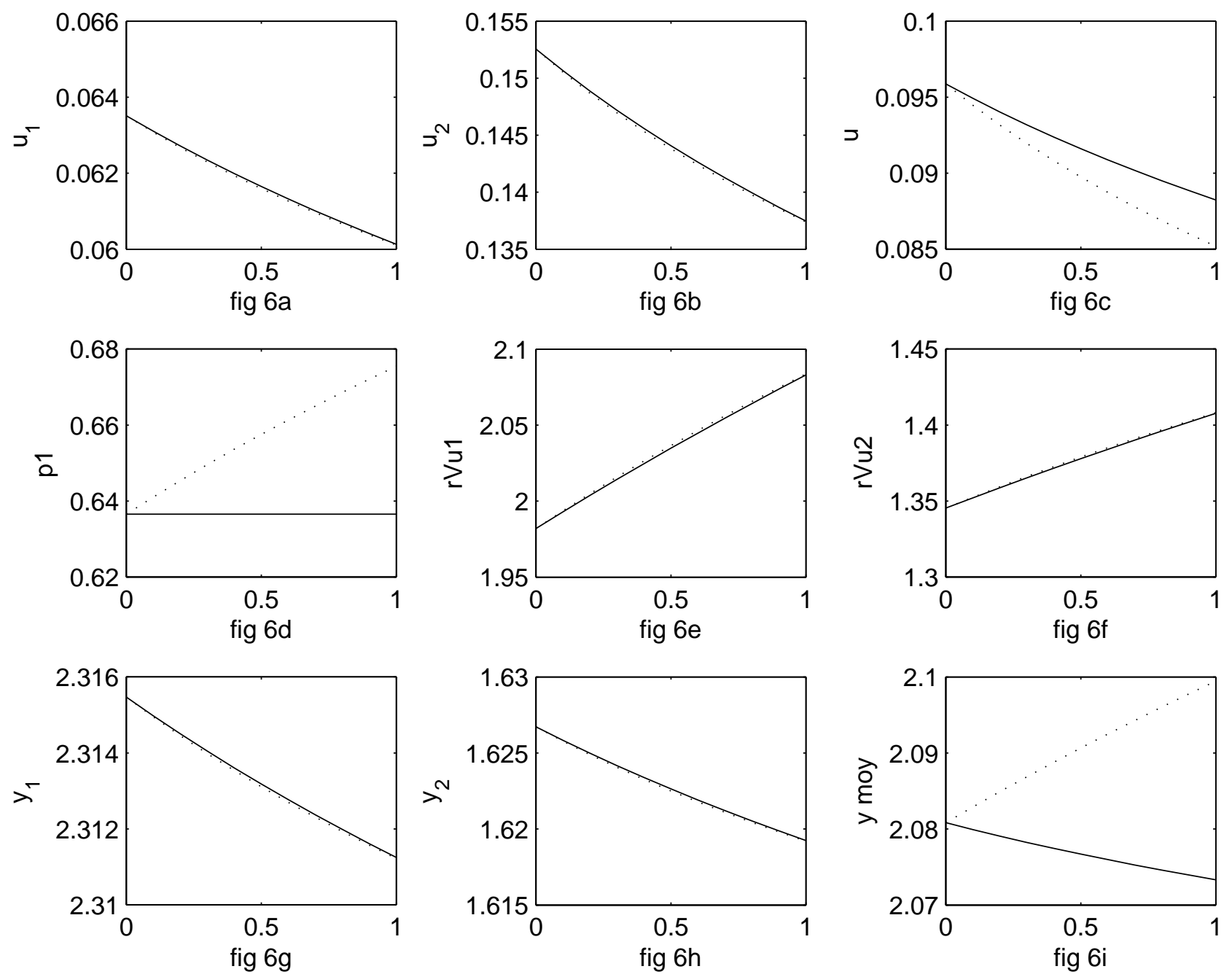

Figure 6 - Effets d'une réforme de la LPE lorsque l'education est exogène (trait continu) et lorsque l'éducation est endogène (traits pointillés). 